San Jose State University

SJSU ScholarWorks

Master's Theses

Master's Theses and Graduate Research

Spring 2013

\title{
Application of Query-Based Qualitative Descriptors in Conjunction with Protein Sequence Homology for Prediction of Residue Solvent Accessibility
}

Reecha Nepal

San Jose State University

Follow this and additional works at: https://scholarworks.sjsu.edu/etd_theses

\section{Recommended Citation}

Nepal, Reecha, "Application of Query-Based Qualitative Descriptors in Conjunction with Protein Sequence Homology for Prediction of Residue Solvent Accessibility" (2013). Master's Theses. 4299.

DOI: https://doi.org/10.31979/etd.em6c-qn2b

https://scholarworks.sjsu.edu/etd_theses/4299

This Thesis is brought to you for free and open access by the Master's Theses and Graduate Research at SJSU ScholarWorks. It has been accepted for inclusion in Master's Theses by an authorized administrator of SJSU ScholarWorks. For more information, please contact scholarworks@sjsu.edu. 


\title{
APPLICATION OF QUERY-BASED QUALITATIVE DESCRIPTORS IN CONJUNCTION WITH PROTEIN SEQUENCE HOMOLOGY FOR PREDICTION OF RESIDUE SOLVENT ACCESSIBILITY
}

\author{
A Thesis \\ Presented to \\ The Faculty of the Department of Chemistry \\ San Jose State University
}

In Partial Fulfillment

of the Requirements for the Degree

Master of Science

by

Reecha Nepal

May 2013 
(C) 2013

Reecha Nepal

\section{ALL RIGHTS RESERVED}

The Designated Thesis Committee Approves the Thesis Titled 


\section{APPLICATION OF QUERY-BASED QUALITATIVE DESCRIPTORS IN CONJUNCTION WITH PROTEIN SEQUENCE HOMOLOGY FOR \\ PREDICTION OF RESIDUE SOLVENT ACCESSIBILITY}

by

Reecha Nepal

APPROVED FOR THE DEPARTMENT OF CHEMISTRY

SAN JOSE STATE UNIVERSITY

May 2013

Dr. Brooke Lustig

Department of Chemistry

Dr. Daryl K. Eggers

Department of Chemistry

Dr. Marc d'Alarcao

Department of Chemistry 


\section{ABSTRACT \\ APPLICATION OF QUERY-BASED QUALITATIVE DESCRIPTORS IN CONJUNCTION WITH PROTEIN SEQUENCE HOMOLOGY FOR PREDICTION OF RESIDUE SOLVENT ACCESSIBILITY}

by Reecha Nepal

Characterization of relative solvent accessibility (RSA) plays a major role in classifying a given protein residue as being on the surface or buried. This information is useful for studying protein structure and protein-protein interactions, and it is usually the first approach applied in the prediction of 3-dimensional (3D) protein structures.

Various complicated and time-consuming methods, such as machine learning, have been applied in solvent-accessibility predictions. In this thesis, we presented a simple application of linear regression methods using various sequence homology values for each residue as well as query residue qualitative predictors corresponding to each of the 20 amino acids. Initially, a fit was generated by applying linear regression to training sets with a variety of sequence homology parameters, including various sequence entropies and residue qualitative predictors. Then the coefficients generated via the training sets were applied to the test set, and, subsequently, the predicted RSA values were extracted for the test set. The qualitative predictors describe the actual query residue type (e.g., Gly) as opposed to the measures of sequence homology for the aligned subject residues. The prediction accuracies were calculated by comparing the predicted RSA values with NACCESS RSA (derived from X-ray crystallography). The utilization of qualitative predictors yielded significant prediction accuracy. 


\section{ACKNOWLEDGEMENT}

First of all, I would like to express my gratitude towards my research advisor, Dr. Brooke Lustig, for guiding me through all of this work. I would like to thank him for his patience, the amount of time he spent with me, and all the opportunities he provided me. I will forever be thankful for his encouragement and his positive attitude when things were not working as expected. I would also like to thank my M. S. thesis committee members, Dr. Daryl Eggers and Dr. Marc d'Alarcao, first for agreeing to be in my committee. Second, for spending time in reading and providing valuable feedback on my thesis. I would also like to thank my parents and my brother for their unshakable believe in me. Finally, I would like to extend my heartfelt gratitude towards my husband, Sailesh Agrawal, without whose help, support and encouragement this work would not have been possible. 


\section{CONTENTS}

List of Abbreviations ...................................................................................................... viii

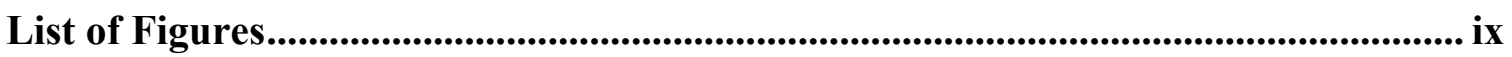

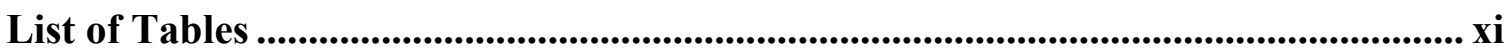

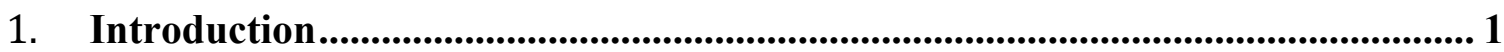

1.1 Protein Structure Prediction Methods ........................................................................... 1

1.1.1 Experimental Approaches.............................................................................. 2

1.1.2 First Approaches in Protein Structure Determination ............................................. 3

$1.2 \quad$ Sequence Entropy ………............................................................................................. 5

1.3 Overview........................................................................................................................ 7

1.4 Organization of the Thesis............................................................................. 8

2. Methods.......................................................................................................................... 9

2.1 Protein Sets and Preparations...................................................................................... 9

2.2 Residue Packing Density .............................................................................................. 13

2.3 Sequence Variability ............................................................................................... 14

2.4 Homology-Based Parameters ...................................................................................... 15

$2.5 \quad$ RSA Calculations ..................................................................................................... 16

2.6 Determination of Qualitative Predictors ....................................................................... 17

2.7 PSI-BLAST Calculations .............................................................................. 19

2.8 Accuracy Calculations................................................................................................... 22

2.9 Aggregate Analysis and Correlation Plots ................................................................... 23

2.10 Frequency Distributions ............................................................................................ 23

2.11 Assimilation of Additional Methods to Improve Accuracy ………………………...... 24

2.11.1 Incorporation of Tertiary Protein Structure Information …………………………... 24

2.11.2 Additional Models Applied .............................................................................. 26

2.11.3 Incorporation of a Categorized Protein Data Set..................................................... 27

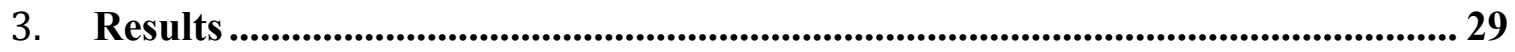

3.1 Characterization of Protein Lists.............................................................................. 29

3.2 Accuracy of Results ................................................................................................... 53

3.3 Outcome of Additional Methods to Improve Prediction Accuracy ……………...... 57

3.3.1 Outcome of Additional Models Applied …………………………………………... 57

3.3.2 Outcome of Categorized Protein Data Set................................................................ 58

3.3.3 Use of All 618 PDB IDs as Training and 215 as Test.............................................. 60 
3.4 Incorporation of the Categories to the Existing Data Sets ....................................... 62

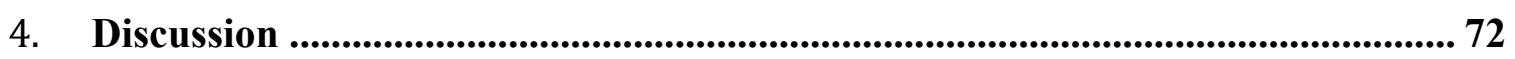

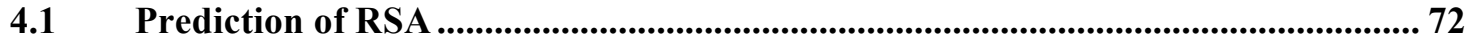

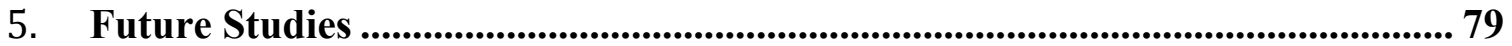

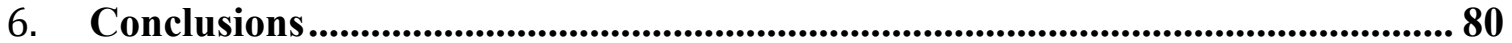

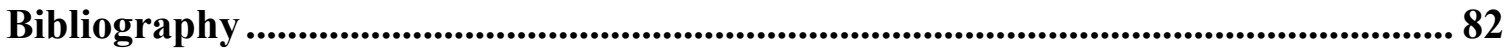

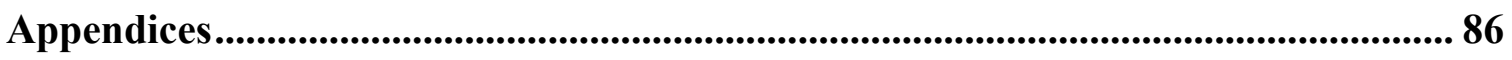

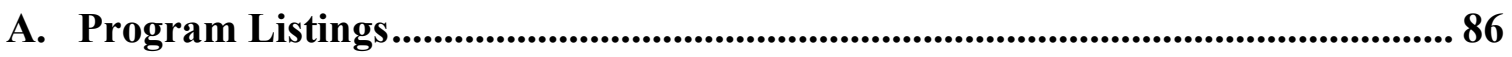

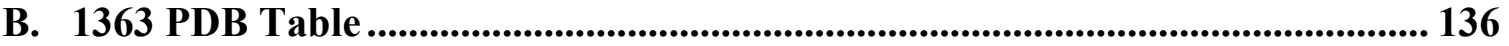




\section{List of Abbreviations}

\begin{tabular}{|l|l|}
\hline Abbreviations & Full Form \\
\hline PDB & Protein Data Bank \\
\hline FSR & Fraction Small Residues \\
\hline FSHP & Fraction Strongly Hydrophobic \\
\hline NSHP & Non-Strongly Hydrophobic \\
\hline FA & Fraction Alanine \\
\hline FG & Fraction Glycine \\
\hline E6 & 6-point Sequence Entropy \\
\hline E20 & 20 term Sequence Entropy \\
\hline RSA & Relative Solvent Accessibility \\
\hline PB & Protein Binding \\
\hline
\end{tabular}




\section{List of Figures}

Figure 2.1 Flowchart of steps involved in the generation of the 1363

training data set based on the list of proteins from Bondugula et al. (2011).

Figure 2.2. Sample regression fit for 73,734 query residues from the

268 training data set.

Figure 2.3: Sample regression fit for 319,551 query residues from the 1363 training data set.

Figure 3.1. Frequency distributions for the characterization of the 268 learning set list of proteins. 31

Figure 3.2. Frequency distributions for the characterization of the 1363

learning set list of proteins.

Figure 3.3. Frequency distributions of entropies and homology-based parameters of the 268 training set list of proteins. 36

Figure 3.4. Frequency distributions of entropies and homology-based parameters of the 268 data set for the two major regions, Region I and Region II. 38

Figure 3.5. Frequency distributions for entropies and homology-based parameters of the 1363 learning set list of proteins.

Figure 3.6. Frequency distributions of entropies and homology-based parameters of the 1363 training data set for the two major regions, Region I and Region II.

Figure 3.7. Frequency distribution of NACCESS RSA values for various RSA ranges for the 268 training data set.

Figure 3.8. Frequency distribution of RSA values for various RSA ranges for the 1363 training data set.

Figure 3.9. Frequency distribution comparison of NACCESS RSA values and predicted RSA values for the 215 test set using the 268 training set.

Figure 3.10. Frequency distribution comparison of NACCESS RSA values and predicted RSA values for the 215 test set using the 1363 training set.

Figure 3.11. Comparison of combined aggregate correlation plots of sequence entropy and other homology-based parameters for the 268 training set. 48 
Figure 3.12. Combined aggregate correlation plots of sequence entropy and other homology-based parameters for the 1363 training set.

Figure 3.13. Density—relative surface accessibility comparison for the 268 training set

Figure 3.14. Density—relative surface accessibility comparison for the 1363 training set...... 


\section{List of Tables}

Table 3.1: Comparison of regression accuracy using manually generated BLAST output calculation and automatically generated BLAST output calculations..... 55

Table 3.2: Summary of regression accuracy for the 12 models tested. ........................... 56

Table 3.3: Regression accuracy table for additional models applied................................. 58

Table 3.4: Protein binding category PDB ID matches between the 618 Hotpatch protein PDB IDs (Petit et al., 2007) and the 268 and 1363 training sets and 215 test set. 59

Table 3.5: Regression accuracy table for protein binding model. 60

Table 3.6: Description of 618 PDB IDs as training and 215 PDB IDs as test regression analysis calculations.

Table 3.7: Regression accuracy table of PDB IDs of the four groups: 1. generic 2. protein binding 3. oligomers 4. and generic without PB and Oligomers used in the regression analysis.

Table 3.8: PDB ID matches between the 618 data set with three data sets $(215,268$, and 1363) for the protein binding and oligomer categories.

Table 3.9: PDB ID matches between the 618 data set with three data sets $(215,268,1363)$ between all categories.

Table 3.10: PDB ID matches between the 618 data set and the three data sets $(215,268$, and 1363) between all categories without the protein binding and oligomer matches.

Table 3.11: Regression accuracy for generic category using common PDB IDs between the 618 data set and the 215, 268, and 1363 data sets for the two regression models.

Table 3.12: Regression accuracy for the protein binding category using common PDB IDs between the 618 data set and the 215, 268, and 1363 data sets for the two regression models.

Table 3.13: Regression accuracy for the oligomer category using common PDB IDs between the 618 data sets and the 215, 268, and 1363 data sets for the two regression models. 
Table 3.14: Regression accuracy for generic category excluding protein binding and oligomer categories using common PDB IDs between the 618

data sets with 215,268 and 1363 data sets for the two regression models..................... 71 


\section{Introduction}

Proteins, polymers of 20 amino acids, are biological macromolecules essential to life that have a variety of functions within each cell. Proteins serve both mechanical (actin in muscle that aids in mobility) and structural (involved in system of scaffolding in cell to maintain shape) functions. Many proteins are enzymes that are involved in vital biochemical processes such as metabolism. In addition to these roles, proteins are also involved in cell signaling, immune responses, cell adhesion, and cell cycle.

\subsection{Protein Structure Prediction Methods}

Protein structure is directly related to protein function. The study of protein structure and function could provide much-needed insight in the role of proteins in vivo. Knowledge of protein structure is fundamental for uncovering mechanisms of actions for various protein functions, exploring protein-protein interaction, predicting protein hydration sites, and characterizing hydrophobic clusters in proteins. Furthermore, structure explorations could facilitate the discovery and development of various drugs, so as to aid in the discovery of solutions to different protein malfunction and protein absence

disorders in humans. Protein structure and functions are studied both experimentally and computationally. 


\subsubsection{Experimental Approaches}

Protein structure determinations have usually involved expensive and timeconsuming methods such as X-ray diffraction analysis, immunohistochemistry, sitedirected mutagenesis, chromatography, and nuclear magnetic resonance (NMR). Processes such as these provide high-quality 3D protein structure determinations with precise accuracy and sensitivity. However these processes can be extremely expensive and time consuming. As a result, the corresponding 3D structures of the majority of proteins have not yet been characterized. In addition, because there can be significant difficulty in characterizing protein structures via X-ray, there is a desire for protein prediction using sequence and/or sequence homology (Dale et al., 2003).

Historically, a vast majority of proteins in the Protein Data Bank (PDB) are determined via X-ray (82\%) with NMR being utilized for most of the remaining structures (Berman et al., 2000). The success rate of a high-resolution 3D protein structure analysis is very low, with only $2-10 \%$ of protein targets resulting in highresolution protein structures (Mizianty and Kurgan, 2011). In order to deduce the correct 3D structure of a protein, it is essential to have a high-resolution crystal structure of the protein. Protein crystal formation is an active field of biological science, and much work is required before the low yield of protein crystallization can be improved. Moreover, there are certain groups of proteins, such as some membrane ribosomal proteins, for which crystal structure deduction is problematic (Gluehmann et al., 2001; Mizianty and Kurgan, 2011). Often these difficulties result from the production of diffraction-quality crystals (Chayen and Saridakis, 2008). The target protein crystallization process can be 
expensive and time consuming because it involves trial and error until the best-quality crystal is obtained.

Interestingly, the use of computational methods in protein crystallization determination can aid in directing the most efficient use of resources in the current coordinated effort to determine high-resolution 3D structures for the whole proteome. One of the primary examples of this is the establishment of the Protein Structure Initiative (PSI), whose main goal is to determine 3D structures of proteins on a large scale (Berman, 2008). This has also greatly improved success rates of structural methods (Graebsch et al., 2010; Savchenko et al., 2003). One offshoot of X-ray structure determination involves a special class of computational modeling that is sometimes referred to as protein homology modeling.

Here, homology involves aligning 2 or more sequences of a known X-ray structure, essentially "stitching" them together, and subsequently optimizing the resulting form. One such homology-modeling program is called MODELLER (Eswar et al., 2006). It implements a process similar to that of $2 \mathrm{D}$ NMR called spatial restraints. In this process, a set of geometrical constraints is used to create a probability density function for the location of each atom in the protein.

\subsubsection{First Approaches in Protein Structure Determination}

Bioinformatics largely involves the characterization of protein structure and function, but it is not necessarily involved in the difficult task of detailed protein structure determination. There is a vast amount of protein sequence data available, and screening 
these sequences would be greatly beneficial in terms of characterizing protein function (Panchenko et al., 2005).

Water plays a major role in biological function. The ability to correctly predict solvent accessibility from a protein sequence could be very useful in characterizing protein interaction and function as well as guiding protein modification and design. The results of the characterization of solvent-accessible surfaces are useful in many protein design and structural biology applications (Petrova and Wu, 2006; Pettit et al., 2007). This includes identification of catalytic and other key functional residues including those found on protein surfaces. This of course augments the restricted number of proteins with extensive 3D structures determined from X-ray and NMR. In addition, solventaccessible surface prediction has garnered attention for its usefulness in the characterization of protein-protein interactions (Porollo et al., 2007). Furthermore, the characterization of solvent-accessible surface has been a standard first approach in determining protein structure. The ability to predict solvent accessibility of a given residue would yield great benefits.

Using the primary amino acid sequence to predict surface-accessible residues has been a standard first approach in structural biology's pursuit to model 3D protein structures. Solving this problem has been of great interest as a testing platform for a variety of machine-learning methods. Protein characterization from sequence data can also be applied to other biological issues such as identification of key core (e.g., strongly hydrophobic) residues (Berezovsky and Trifonov, 2001; Poupon and Mornon, 1999). Such methods hold the potential for increased understanding of the fundamentals of 
protein folding. Methods utilizing sequence information usually use machine-training approaches and have shown accuracy of 70-78\% (Adamczak et al., 2004; Richardson and Barlow, 1999; Rost, 1994;).

Currently, various methods have been reported to yield better prediction accuracy. Among these methods, the following are noteworthy: two-stage and related regression approaches, nearest neighbor method, decision tree methods such as random forest method, and support vector machine learning (Adamczak et al., 2004; Joo et al., 2012; Mizianty and Kurgan, 2012; Pugalenthi et al., 2012). Typically in these types of methods, the test (experimental) relative surface accessibility (RSA) value is predicted based on a particular or constraints model, and consensus predictors are generated via the use of a training data set.

\subsection{Sequence Entropy}

Another structural feature extensively used is the identification of the key core of proteins mostly made up of strongly hydrophobic residues (Berezovsky and Trifonov, 2001; Poupon and Mornon, 1999). The identification of key core residues can help define key constraints in modeling a protein's folding and characterization. Shannon entropies for protein sequences have been used to score amino acid conservation (Koehl and Levitt, 2002; Shenkin et al., 1991; Valdar, 2002). Sequence entropy (Shannon entropy) is the ability of a residue in protein sequence to mutate or change. The correlation of Shannon entropy is greater mutability at a particular amino acid position, the more able an amino acid is able to adapt to a mutation (Hseih et al., 2002). On one hand, the core (hydrophobic) regions are usually evolutionarily well conserved; hence, 
they tend to have low sequence entropy values. On the other hand, the non-hydrophobic residues are usually solvent accessible, and tend to have higher sequence entropy values. The sequence entropy, E20, at some residue position $\mathrm{k}$ is expressed as

$$
\mathrm{S}_{k}=-\Sigma_{j=1,20} P_{J k} \log _{2} P_{j k}
$$

here probability $\mathrm{P}_{\mathrm{jk}}$ at amino acid sequence position $\mathrm{k}$ is derived from the frequency for an amino acid type $\mathrm{j}$ for $\mathrm{N}$ aligned residues. Here, each amino acid out of the 20 canonical represents individual groups.

There have been studies where sequence entropy information has been greatly helpful in characterizing protein-protein boundaries (Guharoy and Chakrabarti, 2005). Koehl and Levitt (2002) described a correlation between thermodynamic and sequence entropy in proteins. The Lustig group calculated two regions for sequence entropy and hydrophobicity of individual residues with respect to the inverse of their respective $\mathrm{C} \alpha$ packing densities (Liao, 2005). Out of these two regions, major region II corresponds to less than $11 \mathrm{C} \alpha$ per $9 \AA$ radius and is principally flat and consistent with the most flexible residues. The majority of the most flexible residues display significant exposure to solvent.

The Lustig group previously reported the addition of a second term that uses the corresponding probability $\mathrm{Pj}$ of an amino acid type $\mathrm{j}$ to correct for random substitution that also does not significantly improve the noise or otherwise change the overall trends of the correlation plots (Liao, 2005). Although sequence entropy alone is not a unique identifier of structural features (Guharoy and Chakrabarti, 2005; Oliveira et al., 2002; Yan et al., 2006), it has shown some potential to illustrate protein-protein interfaces. 


\subsection{Overview}

This thesis summarizes calculations that use a set of homology-based parameters to predict the RSA of protein residues. Thresholds were applied to the RSA values of a given residue where 1 was assigned as being on the surface and 0 as being buried. After reproducing and automating the existing training set of 268 protein sequences, a larger data set comprising 1363 diverse computationally designed proteins (described in Chapter 2) was incorporated in the calculations. BLASTP was used to align all of the proteins with known protein sequences from the database. These results were used to calculate sequence entropy and other homology-based parameters. Packing density was calculated for each residue in the 1363 data set. Entropy, functional parameters, and packing density were determined for each residue in the data set to create an aggregate training set of protein parameters. Once the final aggregate training set was created, statistical software, R, was used to perform linear regression for a total of 12 models comprising various combinations of the functional parameters and entropy values. This linear regression model employs a novel application of a query-based qualitative predictor in conjunction with quantitative protein sequence homology. The training set was used to generate coefficients for linear regression models; once these coefficients were generated, they were applied to the test set to generate estimated RSA values. Finally, as determined from X-ray 3D structure, accuracy was calculated for the prediction. 


\subsection{Organization of the Thesis}

This thesis is organized into 6 parts: Introduction, Methods, Results, Discussions, Conclusion, and Future Studies. The second chapter, Methods, details the calculations and techniques used to come up with the results. This section also explains how linear regression was performed and how different functional parameters including entropy values were generated and used. Chapter 2 also contains a detailed description of how the data sets were generated. The third chapter, Results, includes the key results of the Methods sections. It contains various tables and figures that summarize outcomes of a number of experiments performed. The fourth chapter, Discussion, is focused on a review of available literature. This chapter also notes some areas where calculations could be improved. Chapter 5 provides concluding remarks regarding the methods and results and their context within the literature. Possibilities for future studies are described in the final chapter of this thesis, Chapter 6 . 


\section{Methods}

In order to study protein structure using sequence information, various homology parameters were calculated and their relationship with $\mathrm{C} \alpha$ packing density was investigated. In conjunction with protein sequence homology, a novel application of query-based qualitative predictors was then used to characterize solvent-accessible residues. Out of the three diverse sets of proteins, two (1363 and 268) were used as training sets, and the standard 215 (Naderi-Manesh et al., 2001) was used as a test set.

\subsection{Protein Sets and Preparations}

The 268 protein set, as the name suggests, is made up of 268 diverse protein chains (Mishra, 2010). The goal of recreating calculations and recharacterizing the 268 training set was first to automate the various steps involved. Previously, PDB IDs for the individual 268 training proteins were entered manually in the NCBI website. It was critical to be able to automate this process; with the development and incorporation of a larger training set (1363), it would be an extremely daunting and time-consuming task to manually run BLAST for of each the 1363 PDB IDs. There were instances where NCBI would take much longer ( $>5$ minutes) to run BLAST manually for some PDB IDs. Additionally, it was essential to use the FASTA sequence corresponding to the PDB IDs as the header of the .txt output file for subsequent calculations of various sequence homology parameters and entropies. The automation of this process allowed for ease of incorporation of PDB IDs for additional training and test sets and reduced the number of manually introduced errors. 
The BLAST output file automation was made possible by the python script download_blast.py, and the FASTA sequence was downloaded with the aid of download_blast.py (see Appendix A). Each of these programs can be run in batch, and the resulting BLAST and FASTA files are saved in an output folder.

The 215 set was used as the test set and was the standard 215-protein list from Naderi-Manesh et al. (2001). Earlier calculations of surface-accessible residues have been noted in our previous paper (Rose et al., 2011). The largest set of the three protein sets, 1363, was used as training data. Bondugula et al. (2011) listed a diverse set of 6511 protein domain with 50 designed sequences per domain. The proteins are designed using computational sequence design methods to engineer proteins with desired properties such as increased thermal stability and novel functions.

The 6511 proteins were available in SCOP (Structural Classification of Proteins) format (Bondugula et al. 2011). Since all of the programs and scripts written worked with PDB IDs, the SCOP identifiers of the 6511 set were converted to PDB IDs. SCOP identifiers are made up of 7 characters; the first character is " $d$," the subsequent 4 letters stand for the PDB name, the third part is the DB chain id ("_" if none, "." if multiple), and finally a single-digit integer, if needed, uniquely specifies the domain (“" " if not). All the SCOP identifiers were converted using a python script called SCOPid_to_PDBid.py, which is listed in Appendix A.

Each protein had to be entered in the PDB website to validate its identifier. Again, a python script called common pdbs in the Bondugula set vs PDB library.py (see Apendix A) was written to address this issue. Steps 1 to 4 in Figure 2.1 describe this 
validation method. Out of the original list of 6511 proteins, only 5157 were found in the pbd library, and thus the remaining 1354 PDB names were dropped from the list.

The list of 5157 proteins (see Figure 2.1) was entered in a protein culling service called PISCES (Wang and Dunbrack, 2003). The 5157-protein set was culled for PDB chain identifiers that share $\leq 25 \%$ identity, have a structural resolution of 0.0 to $2.5 \AA$, an R-factor of $\leq 0.3$, and a sequence length of 40 to 10,000 . Protein chains with C $\alpha$-only entries were eliminated, and only protein structures determined via X-ray crystallography methods were selected. The same culling standards were used on the 268 and 215 data sets as well (Mishra, 2010). The final list of all the PDB IDs that that were part of the 1363 training data set is presented in Appendix B. 


\section{Step 1}

i) Open a .txt input file containing all of the 6511 Bondugula PDB IDs, and read in each line

\section{Step 2}

i) Download all of the PDB IDs (June, 2011) present in the PDB library as a .txt file, output a .txt file with all the PDB IDs downloaded

\section{Step 3}

i) Check if the first PDB from the Bondugula set (Step 1) is present in the PDB library (Step 2)

ii) Loop over each of the PDB IDs in the Bondugula set and compare it to the PDB library

\section{Step 4}

i) Print out all of the common PDB IDs from the Bondugula set and the PDB Library ( = 5157 PDB IDs as of June, 2011)

\section{Step 5}

i) Run PISCES culling service with the following criteria: PDB chain identifiers that share $25 \%$, have a structural resolution of $0.0-2.5 \AA$, Rfactor of 0.3 , sequence length of 40-10,000.

ii) Protein chains with $\mathrm{C} \alpha$ only entries were eliminated and only protein structures determined via X-ray crystallography methods were selected

\section{Step 6}

i) Final List of larger training set achieved

ii) Total number of PDB IDs that passed each of the thresholds and requirements = 1366

\section{Step 7}

- 3 PDB IDs (2Q46A, 2CIYA, and 1WTEA) from the 1366 training set had corrupt PDB file. This resulted in exclusion of these three PDB IDs from the list. Final training set $=1363$ PDB IDs

Figure 2.1 Flowchart of steps involved in the generation of the 1363 training data set based on the list of proteins from Bondugula et al. (2011). 


\subsection{Residue Packing Density}

Residue packing density calculates packing density in a residue's native state and is used to measure protein compactness. It is calculated by using X-ray determined $\mathrm{C} \alpha$ coordinates of a given query protein. To calculate protein residue packing density, mmCIF of each protein in the protein sets were downloaded from RCSB PDB (2011).

$\mathrm{X}$-ray crystallography is used to determine the atom co-ordinates information of a protein. Once all the mmCIF files were downloaded, all the $\mathrm{C} \alpha$ coordinates were extracted at each residue position using the python code calculate_density.py (see Appendix A). This program calculates density between any two residues using the following equation:

$$
\operatorname{Dist}(\mathrm{i}, \mathrm{j})=\sqrt{x(i)-x(j)^{2}+\left(y(i)-y(j)^{2}+\left(z(i)+z(j)^{2}\right.\right.}
$$

where $\mathrm{x}, \mathrm{y}$, and $\mathrm{z}$ are the $\mathrm{C} \alpha$ coordinates at that sequence position. Next, the number of $\mathrm{C} \alpha$ atoms within the radius of $9 \AA$ around the residue of interest is calculated. Finally, the packing density at that residue was calculated by determining all other $\mathrm{C} \alpha$ residue positions within $9.0 \AA$ from the C $\alpha$ position of record.

In earlier work conducted by the Lustig group (Mishra, 2010), all the density values were calculated using PERL scripts. In this thesis, all density values were calculated via python scripts using similar logic and calculations. The python program download_mmCIF.py (see Appendix A) downloads each of the mmCIF files for the corresponding PDB ID listed in the input file. On one hand, the mmCIF has some residues whose coordinate values are unavailable; these values were assigned NA. On the other hand, packing density equal to 0 was assigned to the unknown residue such as 
' $\mathrm{X}$ '. Both NA and 0 density values were excluded from frequency plots and correlation plots.

\subsection{Sequence Variability}

Sequence variability for each residue was measured by sequence entropy or Shannon entropy. Sequence entropy is defined as the measure of disorder or randomness in a system. A list of PDB names was acquired for all the protein data sets. Each PDB name was entered in the Basic Local Alignment Search Tool (BLAST 2.2.18+), a protein database program provided by the National Center for Biotechnology Information (NCBI). BLAST searched all the databases available for non-redundant protein sequences using a BLOSUM62 matrix and default gap penalties for each mutational insertion or deletion. Once the PDB name of a protein of interest was entered in the BLASTP website, it is referred to as the query sequence. The query sequence is compared to all of the sequences in the database, referred to as the subject, that are evolutionarily similar to it. The search was performed using default settings except for the Max target sequences setting, which was altered from 100 to 10,000 . The aligned residues were extracted from BLASTP results using a python script labeled blast_to_entropy.py (see Appendix A). BLASTP alignments with bit scores equal to $40 \%$ of the highest bit score were only used for entropy calculations. As noted in a previous thesis, a $40 \%$ cut off seemed to provide an ideal balance between homology and the diversity of sequence variability (Yeh, 2005).

Alternative calculations for sequence entropy were also applied, one involving an application of a 6-term sequence entropy (Mirnya and Shakhnovich, 1999) to the existing 
alignments for all of the data sets. The E6 entropy groups amino acids into six groups and is calculated by:

$$
s_{l}=-\sum_{i=1}^{6} p_{i}(l) \log p_{i}
$$

where $p_{i}$ is the frequency of each of the 6 classes $i$ of residues at position 1 in multiple sequence alignment. The 6 classes of residues are aliphatic (AVLIMC), aromatic (FWYH), polar (STNQ), positive (KR), negative (DE) and special (GP). Once the PDB name of the protein of interest was entered in the BLASTP website, it was referred to as the query sequence. The query sequence was compared to all the subject sequences available in the database that are evolutionary similar to it. The search was performed using default settings except for the Max target sequence setting, which was altered from 100 to 10,000 . The aligned residues were extracted from BLASTP results using a python script labeled blast_to_entropy.py (see Appendix A).

BLASTP alignments with bit scores equal to $40 \%$ of the highest bit score were used only for entropy calculations. A $40 \%$ cut off seemed to provide an ideal balance between homology and the diversity of sequence variability (Mishra, 2010).

\subsection{Homology-Based Parameters}

The development of homology-based parameters was one of the first approaches used to predict solvent accessibility of residues. Once the training and test sets were selected, BLASTP (2010) from Genbank was used to align the sequences. The protein of interest, the query sequence, is aligned with other homologous subject sequences in the 
database, subject sequences that are closely related. Sequence homology parameters for the Lustig group used are 20-term entropy (E20), 6-term sequence entropy (E6), aligned residues that are strongly hydrophobic (FSHP), and aligned small residues (FSR).

Fraction strongly hydrophobic (FSHP) uses strongly hydrophobic residues, VILFYMW (Poupon and Mornon, 1999). FSHP is calculated in the following manner:

$$
\text { Fraction strongly hydrophobic }_{i}(F S H P)=\frac{\text { Number }_{S_{H} P_{i}}}{\text { Total }_{\text {Number of aligned Residues }}}
$$

Where, Number ${ }_{S H P_{i}}$ is the number of strongly hydrophobic residues at sequence position i. Fraction small residues (FSR) refer to residues Gly or Ala and are calculated as follows:

$$
\text { Fraction small residues }_{i}(F S R)=\frac{\text { Number }_{S R_{i}}}{\text { Total } \text { Number of aligned Residues }_{i}}
$$

Fraction Alanine residues (FA) refer, as their name suggests, to residue Ala and are represented by:

$$
\text { Fraction Alanine }(F A)=\frac{\text { Total number of Alanine residues }}{\text { Total number of aligned residues }}
$$

Fraction Glycine residues (FG) represent GLY residues and are represented by:

$$
\text { Fraction Glycine }(F G)=\frac{\text { Total number of Glycine residues }}{\text { Total number of aligned residues }}
$$

\subsection{RSA Calculations}

In this work, residue RSA is calculated with the bioinformatics tool called NACCESS (Hubbard and Thornton, 1993). The NACCESS program calculates accessible surface by rolling a probe of a given size around a van der Waals surface. It also determines a residue accessibility file (.rsa) containing summed atomic-accessible surface areas over each protein residue. The program also normalizes the accessibility of 
each residue calculated as the percent of accessibility compared to the accessibility of that residue type in an extended A-x-A tripeptide format (Hubbard et al., 1991).

The NACCESS RSA values were used as a standard to compare predicted RSA values generated from calculations for this research. RSA values can range from 0 to, very occasionally, 150 . Anything higher than or equal to 20 is regarded as being on the surface and anything less than 20 is considered buried (Carugo, 2000). A binary system was incorporated to support the calculations pertaining to this research. Any RSA value greater than or equal to 20 was assigned a 1, and RSA values less than 20 were assigned a 0 and labeled as buried. Programmatically, the following python scripts were used to calculate RSA values: 1. download_pdb.py, 2. run_naccess.py,

3. RUNNACCESSonUnix.pl, 4. extract_data.py. The corresponding python scripts can be found in the Appendix A section of this thesis.

\subsection{Determination of Qualitative Predictors}

Linear regression is a method used to model relationships between a scalar variable $\mathrm{Y}$ and one or more variables denoted as $\mathrm{X}$. In this method, data are modeled using linear functions, and unknown parameters are estimated from the data. There are two main kinds of variables used in regression analysis: quantitative variables and qualitative variables. On one hand, quantitative variables are expressed as numerical values. Qualitative variables, on the other hand, are categorical expressions. For example, the corresponding barometric height of mercury for a reaction chamber would be $153 \mathrm{~mm}$, whereas pressure could be classified qualitatively as either high or low. Qualitative predictors have been widely used in the social sciences and related fields 
(Hellevik, 2009), but have been relatively unexplored in molecular science. This novel method was used to predict RSA values in this research.

In this section, a simple calculation expressing the application of query-related qualitative predictors for RSA prediction will be illustrated. One of the ways to quantitatively express categorical information is to use indicator variables that take on values, or 0 and 1 (Kutner et al., 2004). Two such delimeters will be expressed here: strongly hydrophobic residues (SHP; VILFYMW) and the remaining non-strongly hydrophobic residues (NSHP). The general model for a first-order linear regression model is:

$$
Y_{i}=\beta_{0}+\beta_{1} X_{i 1}+\beta_{2} X_{i 2}+\varepsilon_{i}
$$

where $Y_{i}$ is a straight line with intercept $\beta_{0}$, slope $\beta_{1}$, and $\varepsilon_{i}$ as residual error function (Kutner et al., 2004). For the SHP and NSHP regression calculations, the 73,675 residue RSA values are fit to the variable $\mathrm{X}_{\mathrm{i} 1}$ corresponding to the E6 value at each residue, $\mathrm{i}$. Here, the two qualitative predictors are $\mathrm{SHP}\left(\mathrm{X}_{\mathrm{i} 2}\right.$ is 0$)$ and $\mathrm{NSHP}\left(\mathrm{X}_{\mathrm{i} 2}\right.$ is 1$)$. The generalized response function can be expressed as:

$$
E\{Y\}=\beta_{0}+\beta_{1} X_{1}+\beta_{2} X_{2}
$$

Substituting the SHP and NSHP value, the fit equation for hydrophobic and nonhydrophobic equation becomes:

$$
\begin{gathered}
E\{Y\}=\beta_{0}+\beta_{1} X_{1} \text { where } X_{2} \text { is } 0 \\
E\{Y\}=\left(\beta_{0}+\beta_{2}\right)+\beta_{1} X_{1} \text { where } X_{2} \text { is } 1
\end{gathered}
$$

The regression for these equations was generated using the programming language, $\mathrm{R}$. Figures 2.2 and 2.3 show the fit for this calculation. To create linear regression plots 
with qualitative predictors, the training data sets' .csv files were used as input files. The input files include one column for NACCESS RSA values and another for the corresponding E6, E, FSR and FSHP values. Now our model can include 20 qualitative predictors that are associated with each amino acid type (for example, A) for all the sequence residues.

\subsection{PSI-BLAST Calculations}

The NCBI website was used to perform PSI-BLAST searches. The PDB names were entered in the search field, and this time around PSI-BLAST was chosen under the algorithm section instead of blastp. Under Algorithm parameter, "Max target sequences" was altered from 500 to 10,000 . After each search result was presented, a second and third iteration were performed. 

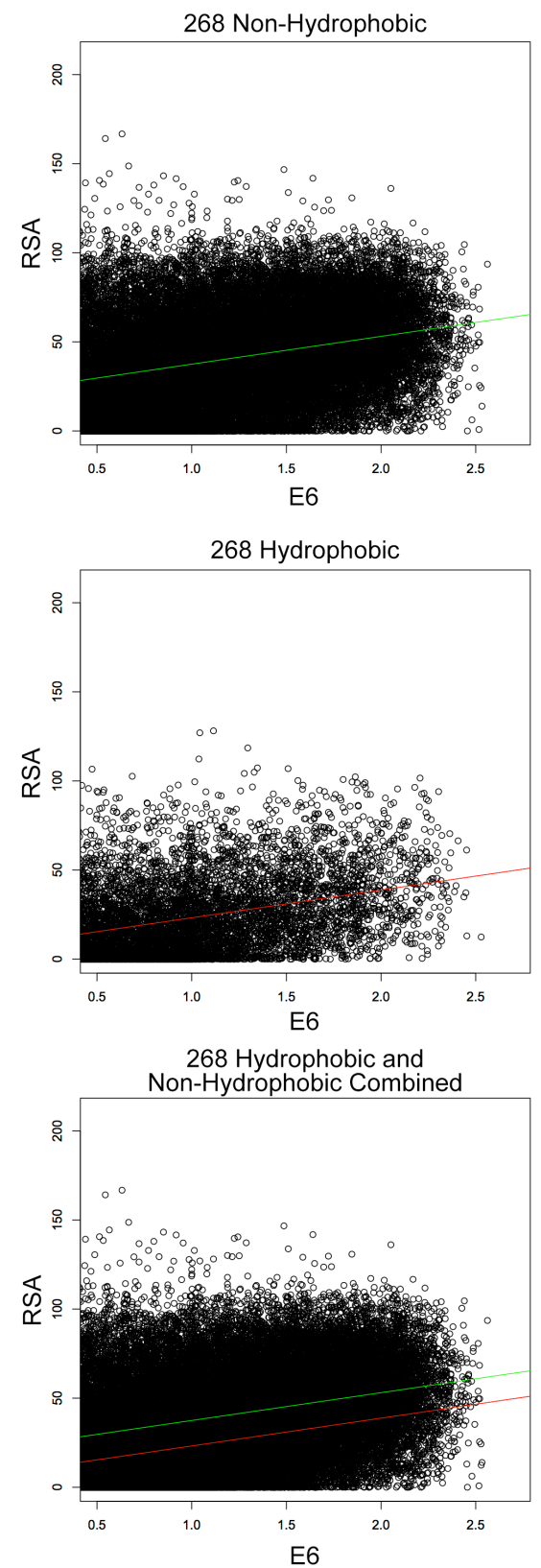

Figure 2.2. Sample regression fit for 73,734 query residues from the 268 training data set. Here, the NACCESS RSA values to a variable term $\mathrm{X}_{\mathrm{i} 1}$ as E6 and the qualitative predictor terms having two values, where $\mathrm{X}_{\mathrm{i} 2}$ is 0 (top) for strongly hydrophobic (SHP) query residues and $\mathrm{X}_{\mathrm{i} 2}$ is 1 (middle) for non-strongly hydrophobic (NSHP) residues, are presented. The slope, 15.6, corresponding to the variable term is the same for both plots, while intercepts are 7.6 and 21.9 for $\beta 0$ and $(\beta 0+\beta 2)$, respectively. The aggregate plot is shown (bottom). 

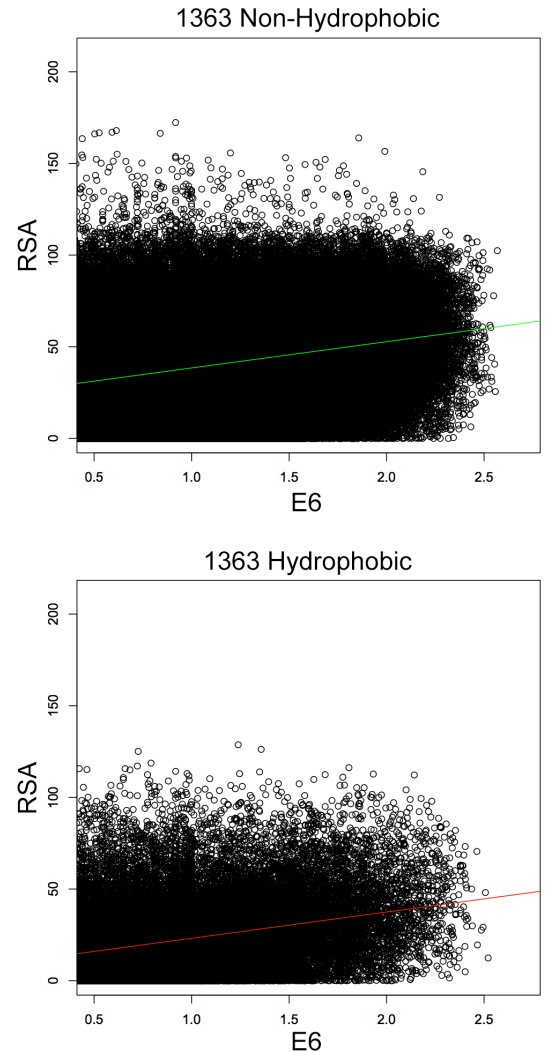

1363 Hydrophobic and

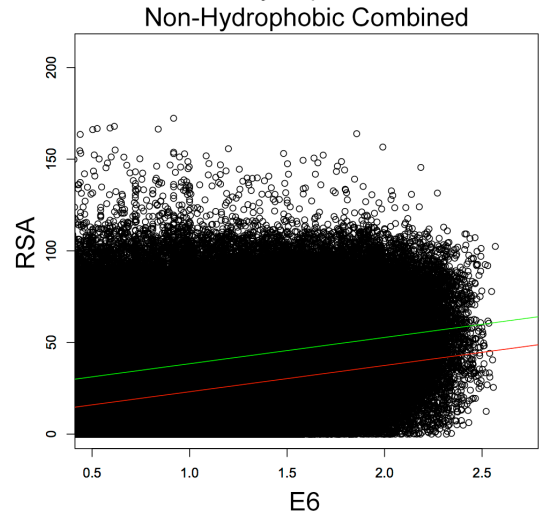

Figure 2.3: Sample regression fit for 319,551 query residues from the 1363 training data set. Here, the NACCESS RSA values to a variable term $\mathrm{X}_{\mathrm{i} 1}$ as E6 and the qualitative predictor terms having two values, where $\mathrm{X}_{\mathrm{i} 2}$ is 0 (top) for strongly hydrophobic (SHP) query residues and $\mathrm{X}_{\mathrm{i} 2}$ is 1 (middle) for non-strongly hydrophobic (NSHP) residues, are presented. The slope, 14.3, corresponding to the variable term is the same for both plots, while intercepts are 8.8 and 24.1 for $\beta 0$ and $(\beta 0+\beta 2)$, respectively. The aggregate plot is shown (bottom). 


\subsection{Accuracy Calculations}

The accuracy for both buried and surface-accessible residues was then calculated by the standard expression of Richardson and Barlow (1999):

$$
\text { Accuracy }=\frac{\text { Number of assignment to correct category }}{\text { total number of assignment made }} * 100 \%
$$

To calculate accuracies for each of the models, principally linear regression was applied to each of the 13 main models used to generate predicted RSA values. These models were made up of various combinations of the two types of entropy (E and E6), and homology-based parameters (FSR, FSHP) with amino acids were used as qualitative predictors. The y-intercept for the line of best fit determined the threshold for the predicted RSA. With the y-intercept for hydrophobic versus non-hydrophobic linear regression line of best fit, a threshold of $>23$ was classified as surface, whereas $\leq 23$ was classified as being buried. This is a result of internal optimization of the test results. The accuracy equation above was then applied in a binary fashion. Any NACCESS RSA that was $\geq 20$ was assigned as 1 meaning on the surface (while 0 was for residues that were buried), any predicted RSA that was $>23$ was also assigned as a 1 . Next, it was noted whether a given residue matched as being on the surface or buried when compared to the NACCESS RSA and predicted RSA. Finally, accuracy was discovered by dividing the number of correctly assigned residues by the total number of predictions made and reported as a percent. Programmatically, the generation of linear regression models, the predicted RSA and accuracy calculations were made possible by R program code $\operatorname{accuracy} \cdot R$ (see Appendix A) 


\subsection{Aggregate Analysis and Correlation Plots}

Once correctly aligned, files were obtained for each of the proteins in the protein sets and additional calculations were conducted. Comparable to procedures listed by Mishra (2010), at each density position the different homology parameters and entropies were averaged. This averaging at each density value was obtained with the help of the python script extract_density_frequency.py (see Appendix A). For example, at the density 4 average, all of the E values for residues that have a density of 4 are present. Similarly, averages were filled out for each of the entropies, FSR, and FSHP for each of the density values. The python script was used to generate a table in .csv format with all the average values. Finally the .csv files were converted to .xlsx format. These averages were used to generate different correlation plots by plotting various homology-based parameters against inverse density.

\subsection{Frequency Distributions}

The homology-based parameters (E20, E6, FSHP, FSR) were aligned together properly together by matching each of the density values with corresponding residue positions. These homology parameters for each of the proteins in a given training or test set were then compiled into a single .csv so that frequency distribution histograms could be generated. As noted in previous work by the Lustig group (Mishra, 2010), each of the density values equal to 0 and NA were eliminated from the list.

Query length for each of the proteins in the lists was calculated with the python script extract_query_length.py (see Appendix A). This program used BLAST output .txt files as input to extract query length. The length of alignment was also generated with 
the help of another python program, extract_record_length.py. For the bit score frequency plot, the frequency of subject proteins at BLAST bit score were generated with the help of extract_bit_score.py.

\subsection{Assimilation of Additional Methods to Improve Accuracy}

\subsubsection{Incorporation of Tertiary Protein Structure Information}

A second-stage prediction method was the use of tertiary protein structure information to study its impact on prediction accuracy. The goal of this research was to study whether protein tertiary structure information from a limited subset of proteins can aid in assigning the solvent-exposed residues of a protein outside the subset. Previous work from the Lustig group at San Jose State University investigated tertiary contact information (Nguyen, 2012). As outlined in his thesis, a protein tertiary contact is defined as a pair of amino acid interactions that are separated by at least 10 residues in the protein primary sequence (Kallblad and Dean, 2004). The atomic distances of these two amino acids need to be less than the sum of the van der Waals radii of the 2 atoms plus $1.0 \AA$ (Kim and Park, 2003). Protein tertiary structures are also critical for protein stability; while secondary structures are usually unstable, tertiary interactions make them more stable (Daggett and Fersht, 2003).

It has been shown that tertiary interactions in a protein are usually buried, well conserved, and more densely packed than other protein residues (Do, S. and Lustig, B. San Jose State University, San Jose, CA. Unpublished work, 2010). Furthermore, tertiary contact information suits secondary protein structure prediction very well in terms of 
sequence entropy, packing density, and RSA values. Therefore it makes sense to utilize tertiary contact information as a second filter in RSA prediction.

Previous research noted that, out of the 268 proteins in the training set, 75 were known to have tertiary contact (Nguyen, 2012). A 95\% confidence interval was applied to the 75 tertiary contact proteins to derive the appropriate tertiary contact threshold. The $95 \%$ confidence interval here implies that, out of all the tertiary contacts presented, $95 \%$ (including false positives) of the lowest-threshold tertiary contact sequence entropy values are correctly predicted as being buried within a protein. This is because tertiary contacts are more conserved when compared to other residues, and most likely to be found buried. Once the threshold was established, it was applied to the entire 215 test set. This calculation was carried out by dividing each of the proteins into a separate .csv file that included all of its residues. For example, the first protein in the 215 set is 119LA, which has 162 residues; therefore 119LA.csv would have 162 residues present in it. Following this, a matrix was created. In this example, the matrix was 162 by 162 . For each possible position in the matrix, entropy was averaged for the two residues involved (column residue and row residue). If the average entropy of any two residues was greater than the threshold value, then those two residues were predicted to be on the surface, denoted by a 1 ; otherwise, the position was predicted to be buried and assigned a 0 . This information was applied to the predicted RSA values obtained from linear regression data. Anytime, a given residue position was predicted to be on the surface in the tertiary contact matrix, the linear regression data was altered to match the prediction of the tertiary filter. Performing the calculations manually—checking each and every residue 
assigned a 1 in the matrix and referring back to the prediction Excel file- would have been not only challenging, but also prone to many errors. Thus, to address this issue, two R scripts were written: tertiary_contact_filter $2 . R$ and accuracy_with_filter_2.R (see Appendix A). The first program, tertiary_contact_filter 2.R, takes entropy data from multiple residues of a given protein and creates a filter matrix. The second program, accuracy_with_filter_2.R, imposes the threshold to each matrix value and recalculates solvent accessibility accuracy. Contrary to our initial hypothesis, the incorporation of tertiary information in the prediction model did not improve accuracy.

\subsubsection{Additional Models Applied}

Upon further investigation of individual protein accuracies, it was noticed that the smallest residues, alanine and glycine, were the most mispredicted amino acid residues. The small residue fraction was represented as a model in conjunction with the 20 amino acids as a qualitative predictor. However, the initial 11 models were missing fraction strongly hydrophobic (FSHP) in combination with amino acids as qualitative predictors. FSHP + AA was added as a new model. The results of this calculation are presented in Table 3.4 in the Results Section of this thesis.

Fraction Alanine (FA) was the first tested on the regression models. For the new model, FSR was replaced with FA. The results of this test are presented later in this thesis. Again, comparable to FA, model FG also replaced FSR as a model, and accuracy for all the models with FSR replaced with FG were recalculated. The results of this calculation are presented in Table 3.4. Finally, it was not sufficient to just substitute FSR 
with either FG or FA. As a third test to this principle, FSR was swapped with FG + FA; these results are also presented in Table 3.4.

\subsubsection{Incorporation of a Categorized Protein Data Set}

An additional approach that incorporated a new data set was used to study any further impact on the accuracy calculations. In a previous study conducted by Pettit and co-workers (2007), a set of 618 proteins categorized to 15 different chemical groups was investigated by a HotPatch study. HotPatch is a statistical analysis system that finds unusual patches on the surface of proteins and computes just how unusual they are (called patch rareness), as well as the functional importance of each patch. The set of 618 proteins are divided into 12 different groups: proteases, hydrolases, kinases, transferase, oxidoreductases, catalytic general, DNA/RNA interacting, negative ion binding, smallmolecule interacting, carbohydrate interacting, lipid interacting, and positive-metal ion binding. An individual PDB ID from the 618 set could belong to any one of these groups or multiple groups. For the purpose of this study, the 12 original groups were split into three simpler groups: oligomers, protein binding, and generic without oligomers and protein binding. Notably protein binding refers to proteins that transiently bind to other proteins.

The main goal of this experiment was to implement the group information to both the test set and the training set to observe if any progress would be made in the accuracy numbers. There were multiple sub-sets of calculations carried out with this principle, all of which are described in the following subsections of this thesis. 
The first group of proteins investigated was protein binding. The PDB IDs from the 268 training set, 1363 training set, and 215 test set all shared common PDB IDs with the 618 set. A group of PDB IDs from the 268 set that overlapped with the protein binding group was selected; similarly, the PDBs in common between the 215 and 618 protein binding groups were also chosen. 


\section{Results}

\subsection{Characterization of Protein Lists}

The characterization of protein lists based on the method of structure determination, resolution $\mathrm{R}$-factor, free $\mathrm{R}$ value, protein length, and alignment length for the 268 training set has been presented in an earlier study (Mishra, 2010). For the 268 training set, frequency distributions and correlations found in the previous studies were extracted from manually derived BLAST output files. In this section, results from the automated BLAST output files are presented for comparison and validation purposes. The newly developed training set, 1363, has also been characterized using frequency and correlation plots in this section.

The automated BLAST-generated frequency distributions for the characterization of the 268 training set are shown in Figure 3.1. A frequency plot of length of query protein length with regard to the frequency of occurrence is presented in Figure 3.1A. The highest and second highest peaks are represented by a frequency of 39 at a 350protein length and a frequency of 38 for 200-protein length. The histogram distribution appears to be distorted and skewed right; the right tail of the graph is considerably longer than its left tail. The mean value of this histogram was at 283 , and the mode was presented at 340. The length of protein ranges from 50 to 950 ; however, the majority of the proteins (95.5\%) have lengths between 100 and 600 .

Figure 3.1B displays a histogram for density of query residues as a function of frequency of query residues. The mean and mode for this distribution are 17.5 and 15 , respectively. The histogram has normal distribution, with $95.6 \%$ of data points appearing 
within the intervals of 7 and 22. The highest frequency of query protein density, 6467, occurred at a density value equal to 15 . This corresponds to a normal Gaussian-like distribution.

The number of alignments as a function of frequency of query residue distribution plot is presented in Figure 3.1C. The number of alignment ranges from 200 to slightly over 2000 for the frequency of query proteins. The maximum for the number of alignments is at 1200 alignment for the frequency of 104. The shape of the distribution is skewed slightly left, with $69.0 \%$ of alignments hovering from 1000 to 1200 . The mean, mode, and median of the distribution are at 962.55, 1000, and 1000, respectively.

The distribution plot of the BLAST bit score (Figure 3.1D) displays a rightskewed pattern, as expected for such a distribution (Liao et al., 2005). The maximum BLAST bit score occurs at the value of 1894 , with the minimum at 29 . The bit score of 100 is the highest, occurring at the frequency of 88,198 . The mean BLAST bit score is 224 , the mode is 37 , and the median is at 164 .

The goal of re-characterizing the 268 training set was to compare and validate the automated BLAST-generated output performed in previous work by the Lustig group (Mishra, 2010). All four of the distribution plots (length of proteins, density, number of alignments, and BLAST bit score) are almost identical to the manually generated BLAST output calculations. Both sets of the distribution plots have the same maxima, minima, general trend of the histogram, and distribution. This validates the reliability of the python program download_blast.py to automatically download BLAST files from NCBI 
website and shows that the outputs are comparable to manually downloaded BLAST files.

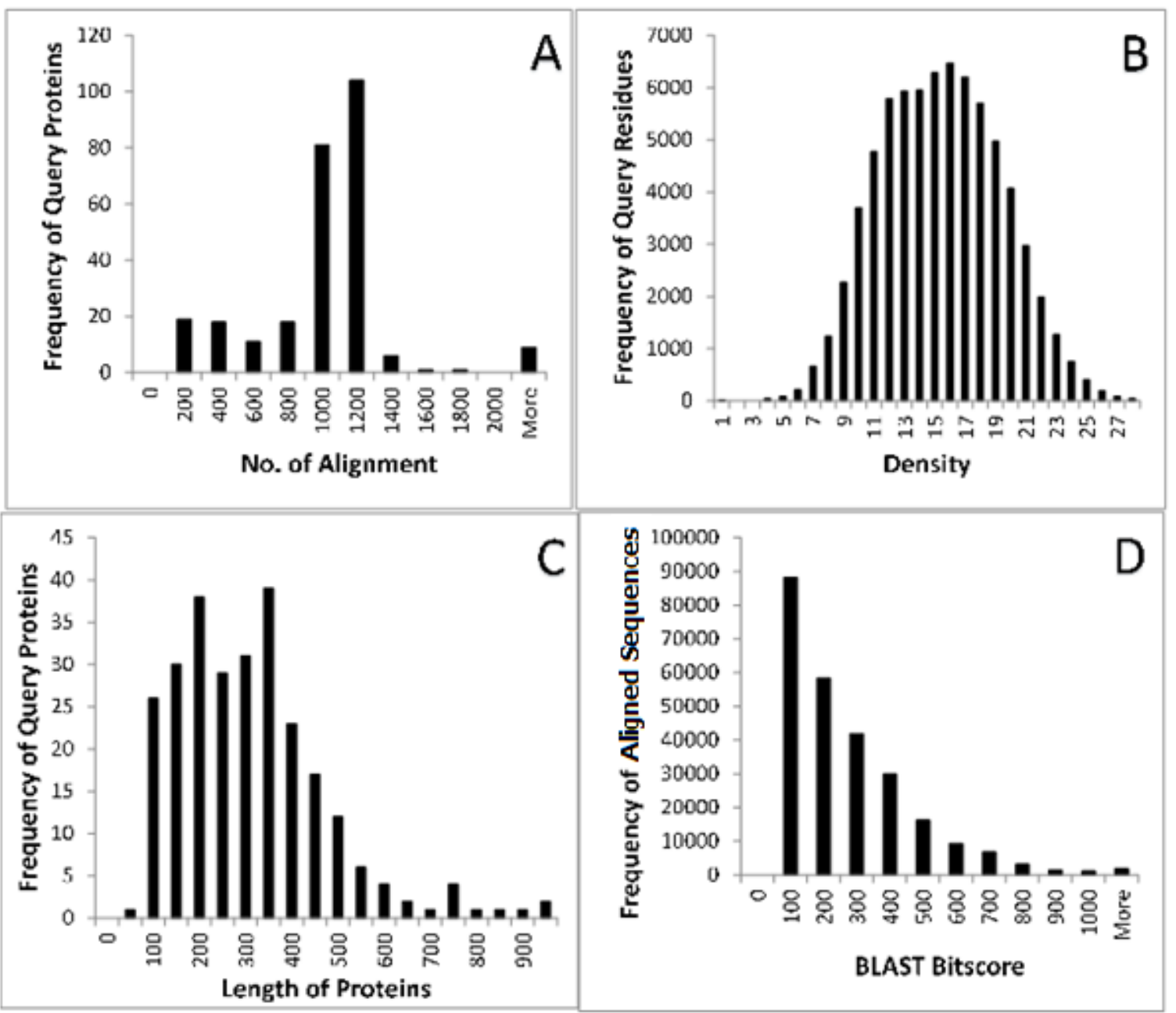

Figure 3.1. Frequency distributions for the characterization of the 268 learning set list of proteins. The 268 proteins in the list have a total of 73,734 query residues, and a total of 257,963 aligned subject protein sequences were used for these calculations. A. Frequency of query residues with respect to length of each protein of the 268 learning set. B. Frequency of 73,734 query residues with respect to each packing density. C. Frequency of query proteins was plotted against a number of alignments obtained from NCBI BLASTP outputs for the learning set. D. Frequency of 257,963 aligned subject sequences with respect to BLAST bit scores. 
Figure 3.2 displays the frequency characterization of the 1363 training set. Since the BLAST output for the manual download and automated download were similar, 1363 BLAST files were automatically downloaded with the aid of down_blast.py. The frequency plot for the 1363 training set (Figure 3.2A) also has length of query protein with respect to frequency of query proteins. This histogram displays a slightly lefttruncated normal distribution. The most frequent query protein length at 150 is shown at a frequency of 281 . The mean of the query length is at 243.8 , and the mode of the distribution is 129 . Comparable to the protein lengths of the 268 training set, the protein length for the 1363 training data set also ranges from 50 to 950 , and the majority of proteins $(95.5 \%)$ have lengths between 100 and 550 .

The frequency of query residues versus density histogram (Fig 3.2B) displays a Gaussian-like distribution. Density of 14 seems to be the most frequent density value at 50,833; however, densities 12 and 16 also flag as close values at 50,024 and 50,457, respectively. It is also evident from the figure that the majority, at $96.2 \%$, of data points occur at densities between 8 and 23 .

The number of alignments associated with the query proteins of the training set list (Figure 3.2C) ranges from 0 to 2000 or more. The maximum number of alignments occurs with a frequency of 391 at a sequence alignment of 1000 for 1KJQA. The bit score distribution plot is right-skewed, as expected (Liao et al., 2005). It indicates a BLAST bit score maximum at 100 occurring 500,000 times. 


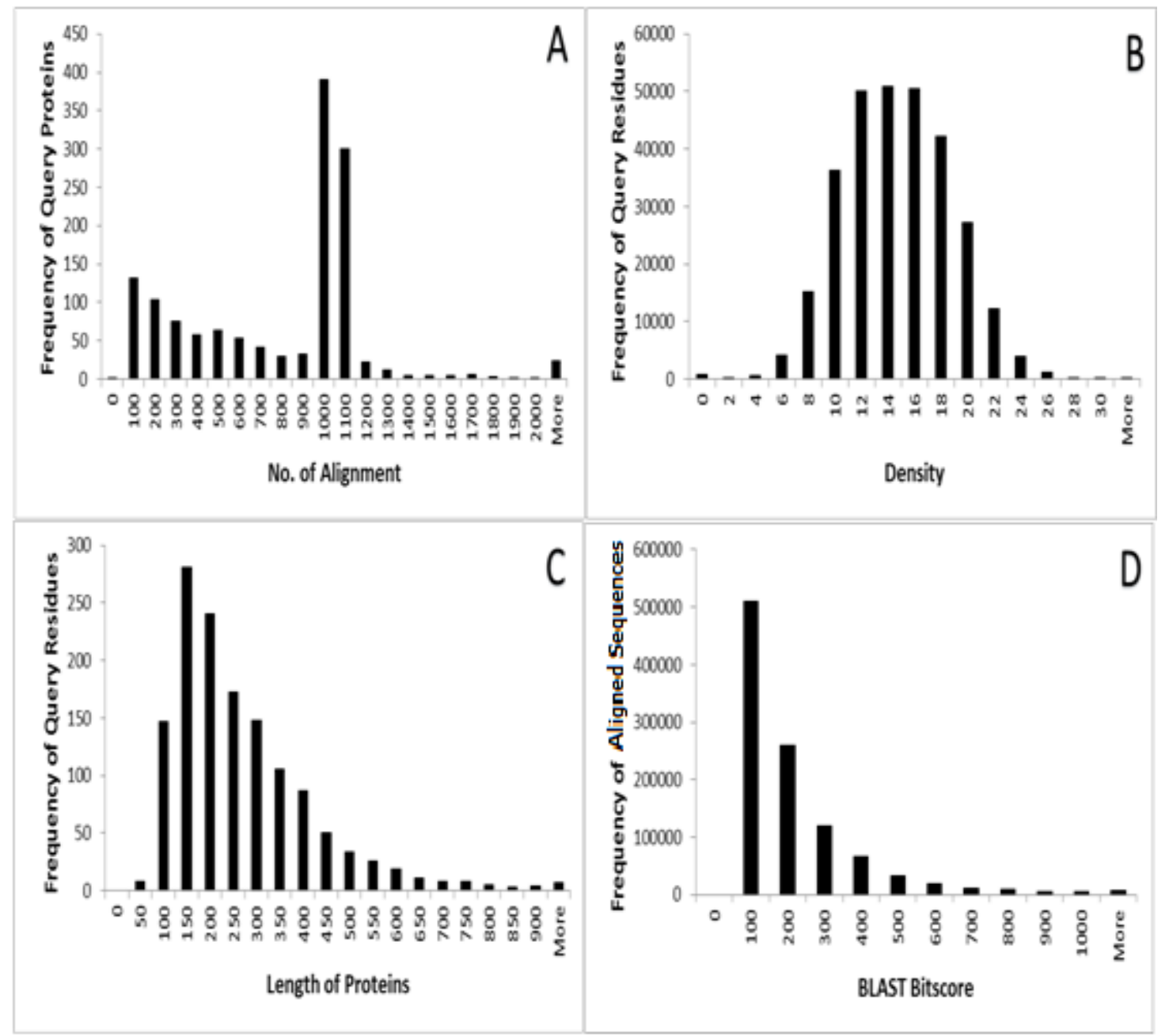

Figure 3.2. Frequency distributions for the characterization of the 1363 learning set list of proteins. The 1363 proteins in the list has a total of 319,551 query residues and total of $1,055,920$ aligned subject protein sequences were used for these calculations. A. Frequency of query residues with respect to length of each protein of the 268 learning set. B. Frequency of 318,840 query residues with respect to each packing density. C. Frequency of query proteins was plotted against a number of alignments obtained from NCBI BLASTP outputs for the learning set. D. Frequency of 1,055,920 aligned subject sequences with respect to BLAST bit scores. 
Figure 3.3 represents the histograms for frequency distributions for entropies and homology-based parameters of the 268 training set of proteins. A total of 73,724 query residues and a total of 257,963 aligned subject protein sequences were used. Figure 3.3A presents the distribution of the calculated 20-point entropy (E20) value as a function of frequency of query proteins. An entropy value of 0 occurred at the highest frequency, with a total of 10,239 residues having this entropy value. The average entropy value for the residues was 1.10 , while the median was 0.972 . The histogram indicates multimodal characteristics. The maximum calculated entropy value was 3.895 . The lower entropy value represents a well-conserved residue. The fact that more than half (51.3\%) of the residues have entropy values between 0 and 1 indicates that half of the residues are well conserved in the 268 training data set.

The distribution of 6-point entropy (E6) as a function of frequency of query residues is presented in Figure 3.3B. Similar to E20 distribution, the highest frequency of entropy values were observed at E6 $=0$. However, the average E6 value at 0.64 was much lower than the E20 average (1.1). The median for this distribution was at 0.397 E6 value. Comparable to the E20 distribution plot in 3.3A, the E6 distribution also appeared to be multimodal. The maximum E6 value was 2.562 .

Figure 3.3C presents the frequency of fraction small residues (FSR) as a function of frequency of query proteins. The mode of this distribution was at FSR $=0$. The average FSR calculated value was 0.1637 , while the median FSR value was 0.006 . The FSR value of 0 occurred at the highest frequency of 31,420 residues. The FSR distribution plot is also right skewed, with slight increases in frequencies of $1,1.1$, and 
$>1.1$. Finally, Figure 3.3D is the frequency plot of fraction strongly hydrophobic residues (FSHP) as a function of frequency of query residues. The mean, mode, and median of this distribution were $0.3247,0$, and 0.0271774 , respectively. Here $59.06 \%$ of residues observed have FSHP values between 0 and 0.1 . For the distributions in Figures $3.3 \mathrm{C}$ and 3.3D, there are clear decoupled components, which has been consistently noted with the manually generated BLAST output calculations (Mishra, 2010) and automated 268 distributions. 


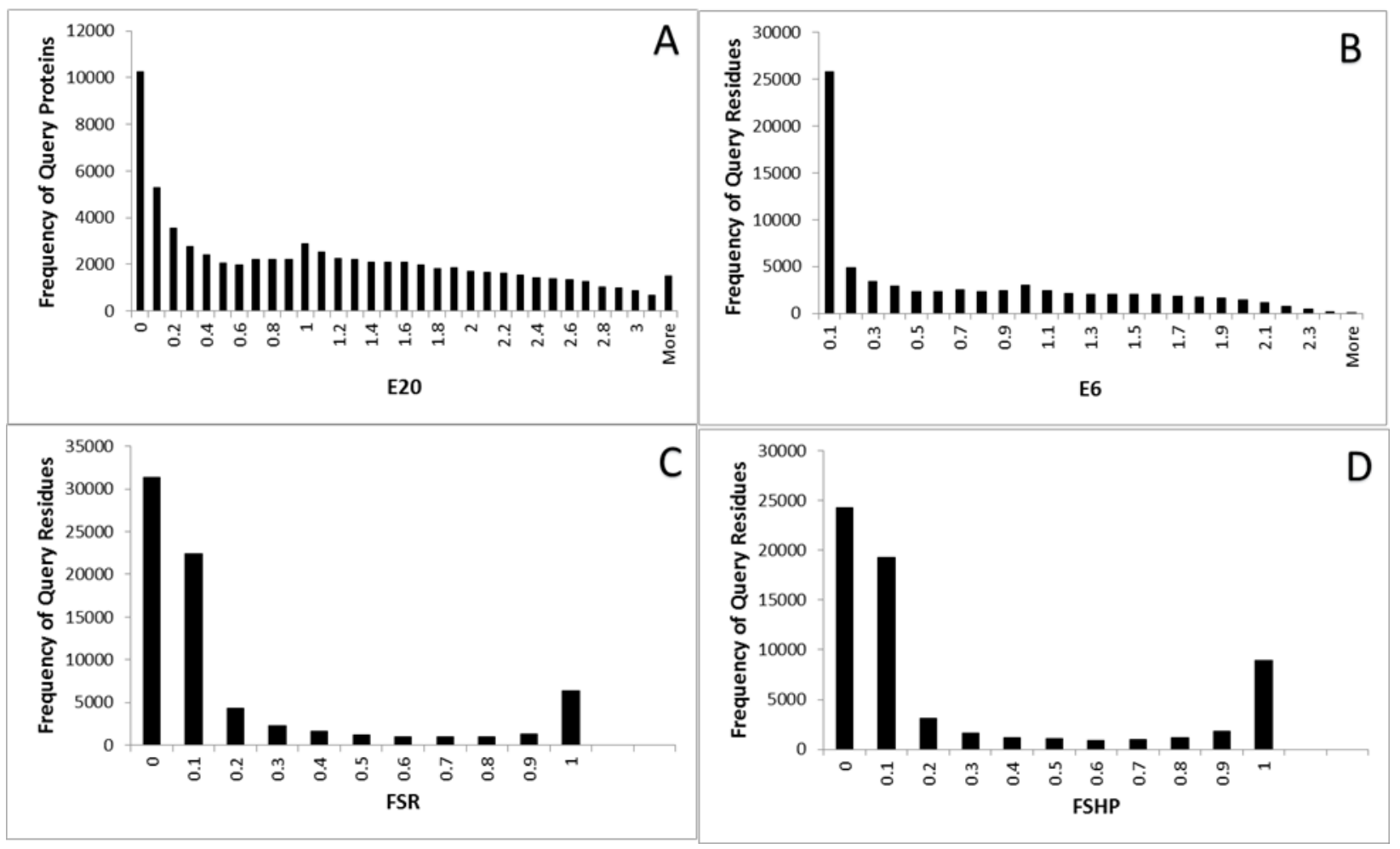

Figure 3.3. Frequency distributions of entropies and homology-based parameters of the 268 training set list of proteins. The 268 proteins in the list has a total of 73,734 query residues and total of 257,963 aligned subject protein sequences were used for these calculations. A. Frequency of query residues with respect to entropy values of the 268 learning set. B. Frequency of query residues with respect to E6 values of the 268 learning set. C. Frequency of query residues with respect to fraction small residues (FSR). D. Frequency of query residues with respect to fraction strongly hydrophobic residues (FSHP)

Figure 3.4 shows the frequency distributions of entropies and homology-based parameters of the automated 268 training set for the two major regions, Region I and Region II. Figure 3.4A presents the E20 distribution for the two major regions, and 3.4B presents the E6 distributions for the two regions. It is observed that at lower entropy (0 and 0.3 ) the majority of residues have RSA value less than 20, whereas at higher entropy 
most of the residues have RSA $\geq 20$ for both E20 and E6 distributions. The frequency distribution of FSR for the two regions peaks at low FSR value (0 and 0.1$)$ and high FSR values ( 1 and 1.1), while the distribution flattens out in the middle ranges. This indicates that FSRs are found both in the core and surface of the protein. The highest frequency are observed at FSR of 0 .

Finally, Figure 3.4D presents the distribution of the 268 training set for the two major regions and shows bimodal characteristics. An FSHP value of 0 indicates the absence of strongly hydrophobic residues. The majority of residues with FSHP $=0$ correspond to an RSA $\geq 20$; this was expected because during protein folding most of the hydrophobic residues are buried. When the FSHP $=1$ or 1.1 , the majority of the query residues have an RSA value of less than 20 . The findings and trends of the automatically generated BLAST output homology parameters showcase similar trends as reported for the manual BLAST output calculation (Mishra, 2010). 

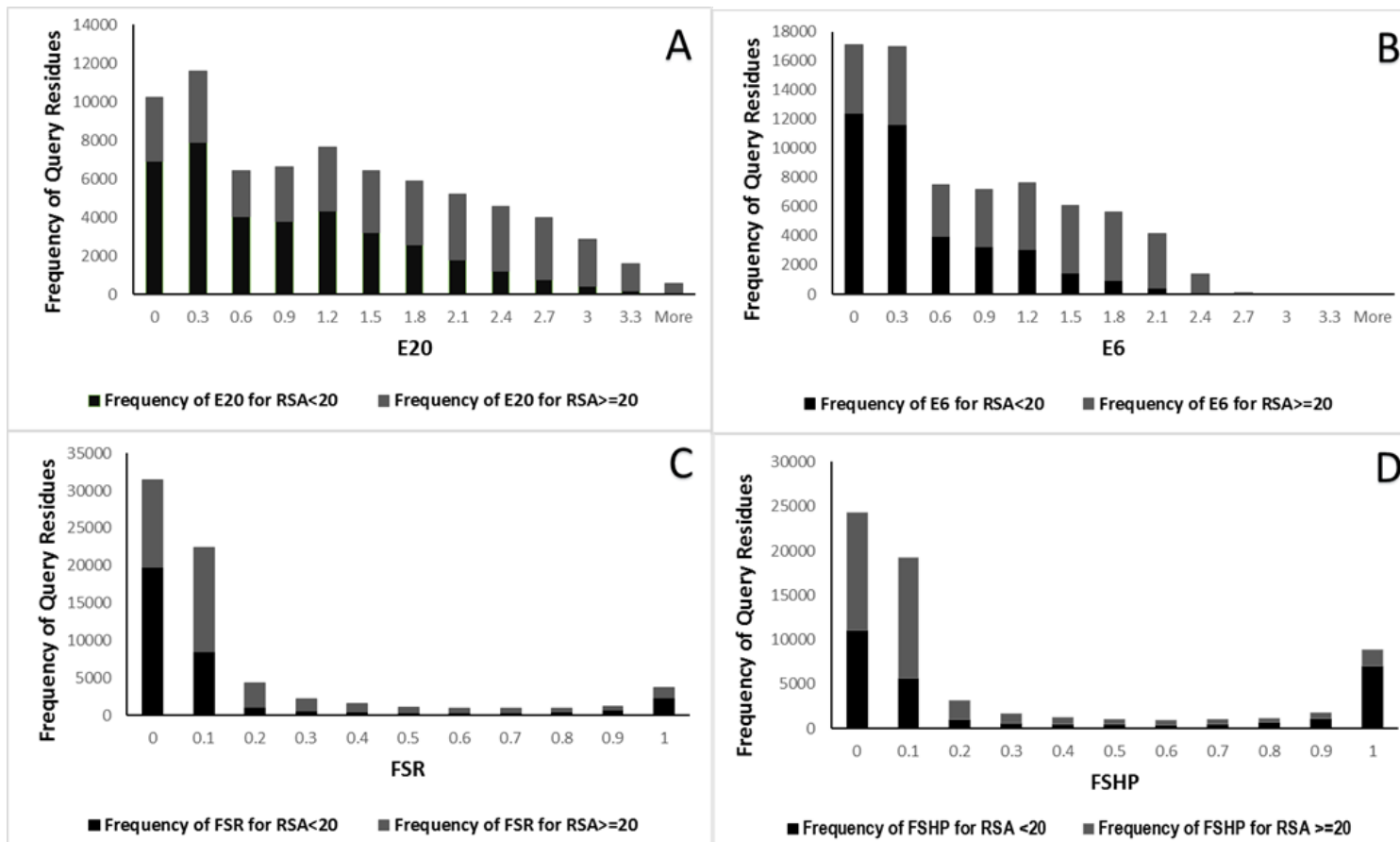

- Frequency of E6 for RSA $<20$

In Frequency of E6 for RSA $>=\mathbf{2 0}$

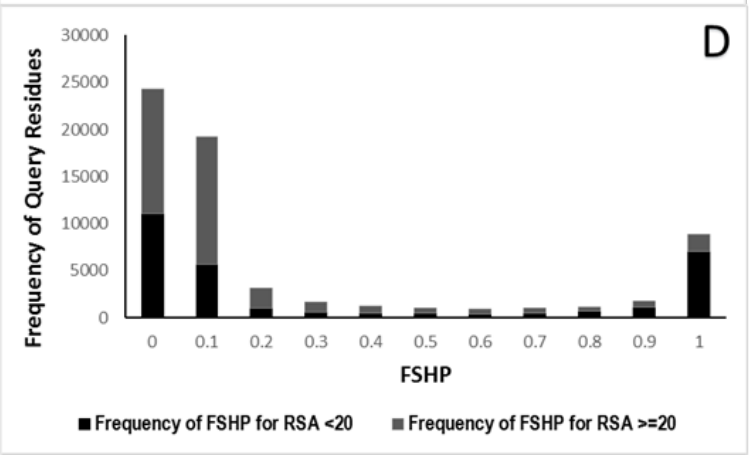

Figure 3.4. Frequency distributions of entropies and homology-based parameters of the 268 data set for the two major regions, Region I and Region II. A total of 73,734 residues were divided into Major Region I $(\mathrm{RSA}<20)$ and Major Region II $(\mathrm{RSA} \geq 20)$. A. E20; B. E6; C. FSR; D. FSHP. 
The histograms for E20, E6, FSR, and FSHP residue occurrence pertaining to the 1363 training set are presented in Figure 3.5. A total of 319,551 query residues were used to generate the distribution histograms. In Figure 3.4A, E20 is plotted as a function of frequency of query residues; the maximum E20 value is at 3.942, and the minimum is at 0 . The distribution of E20 appears to form two clusters, and this suggests two separate normally-distributed populations. The highest frequency for both of these populations occurs at an E20 value of 0, with the frequency of 66,199 (20.7\% of residues have an E20 value of 0). The maximum frequency of the second cluster appears to have an E20 value of 1 with $4.9 \%(15,576)$ of residues.

Figure 3.5B shows the frequency distribution of E6 for 319,551 query residues from the 1363 training data set. The E6 plot also displays similar shape and bimodal distribution as the 268 training set.

The FSR distribution plot as a function of frequency per query residue (Figure 3.5C) does not display patterns similar to those of the E20 and E6 distribution plots. The mode for FSR occurs at 0.0 with $51 \%$ of the residues at frequency of 162,822 . Residues with an FSR value of 0 indicate an absence of small residues, whereas an FSR value of 1 indicates a high number of FSR (A and G). The FSHP distribution plot in Figure 3.5D also shows FSHP mode at zero, with $40 \%$ of the residues $(126,484)$ displaying an FSHP value of 0 . An FSHP value of 0 indicates an absence of substituted strongly hydrophobic residues. 


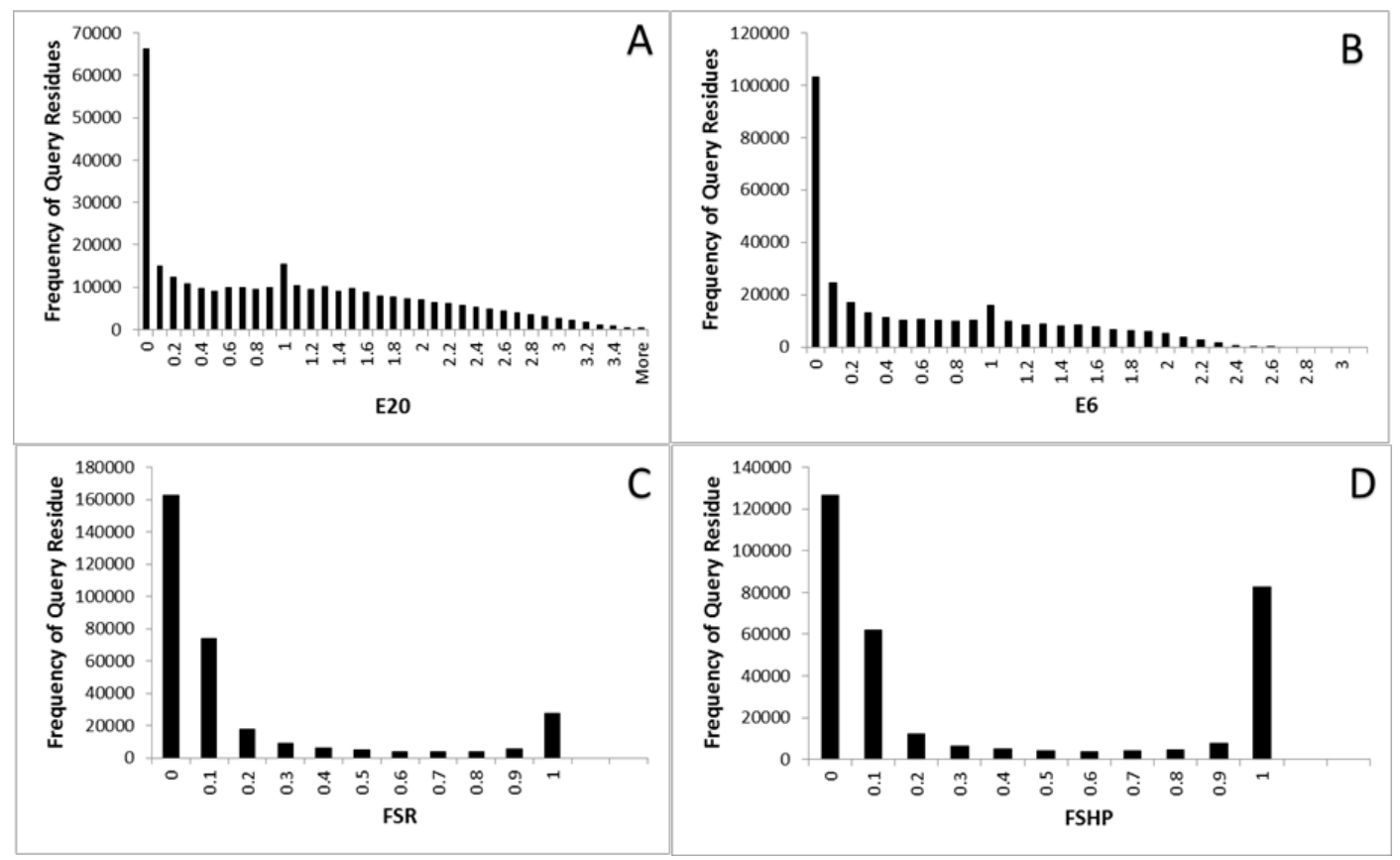

Figure 3.5. Frequency distributions for entropies and homology-based parameters of the 1363 learning set list of proteins. The 1363 proteins in the list have a total of 319,551 query residues, and total of 1,055,920 aligned subject protein sequences were used for these calculations. A. Frequency of query residues with respect to entropy values of the 1363 learning set. B. Frequency of query residues with respect to E6 values of the 1363 learning set. C. Frequency of query residues with respect to fraction small residues (FSR). D. Frequency of query residues with respect to fraction strongly hydrophobic residues (FSHP).

Similar to Figure 3.4, Figure 3.6 presents the frequency distribution of entropies and homology-based parameters, but for the 1363 training set for the two major regions, Region I and Region II. Figure 3.6A shows the E20 distribution for the two major regions, and 3.6B shows is the E6 distributions for the two regions. Similar to the E20, E6, FSR, and FSHP two-region distributions observed for the 268 training set (Figure 3.4), Figure 3.6 also presents similar trends and distribution plots. 


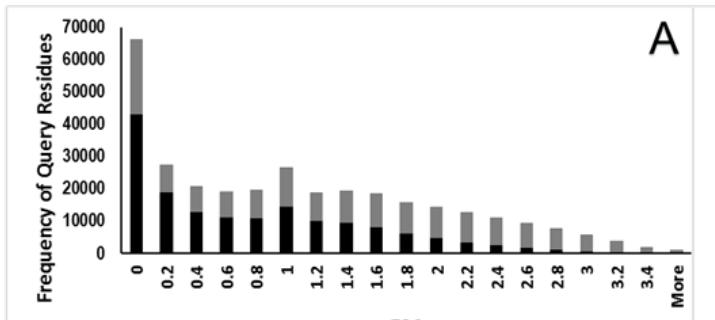

E20

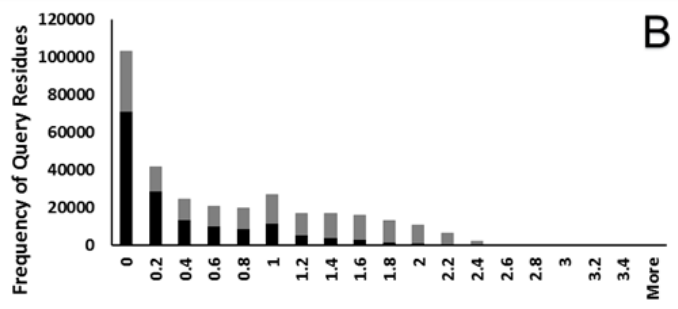

- Frequency of E20 for RSA $<20$ Frequency of E20 for RSA $>=20$

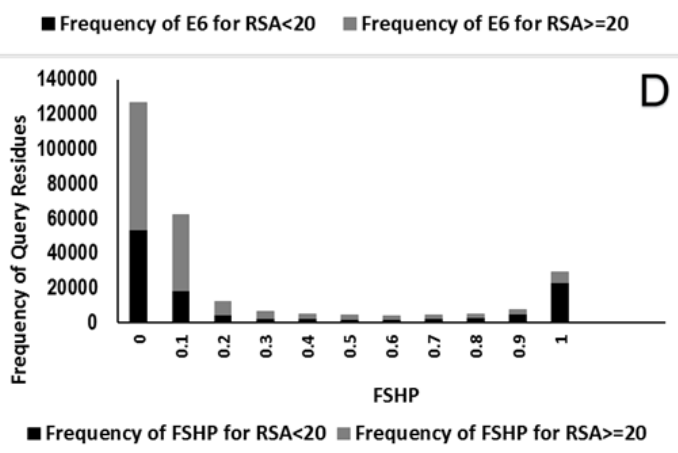

- Frequency of FSR for RSA $<20$ Frequency of FSR for RSA $>=\mathbf{2 0}$

- Frequency of FSHP for RSA $<20$ - Frequency of FSHP for RSA $>=20$

Figure 3.6. Frequency distributions of entropies and homology-based parameters of the 1363 training data set for the two major regions, Region I and Region II. A total of 1,055,920 residues were divided into Major Region I $(\mathrm{RSA}<20)$ and Major Region II $(\mathrm{RSA} \geq 20)$. A. E20; B. E6; C. FSR; D. FSHP.

Figure 3.7 presents the frequency distribution of NACCESS RSA values for 73,734 query residues of the 268 training data set. The mode of the distribution is at a NACCESS RSA value of 10 , with $28.2 \%$ of residues $(20,815)$ displaying this value. Any residue with a NACCESS $\geq 20$ is characterized as being on the surface, whereas residues with a NACCESS value $<20$ are considered buried. Here in this distribution, the mode is 10, which indicates that a significant number of buried residues have a NACCESS value of 10 . There are 28,256 residues with a NACCESS RSA of $<20$, indicating that $38.3 \%$ of 
residues in the 268 training set are buried by X-ray. The remaining $61.7 \%$ of residues with NACCESS RSA values $\geq 20$ are branded to appear on the surface.

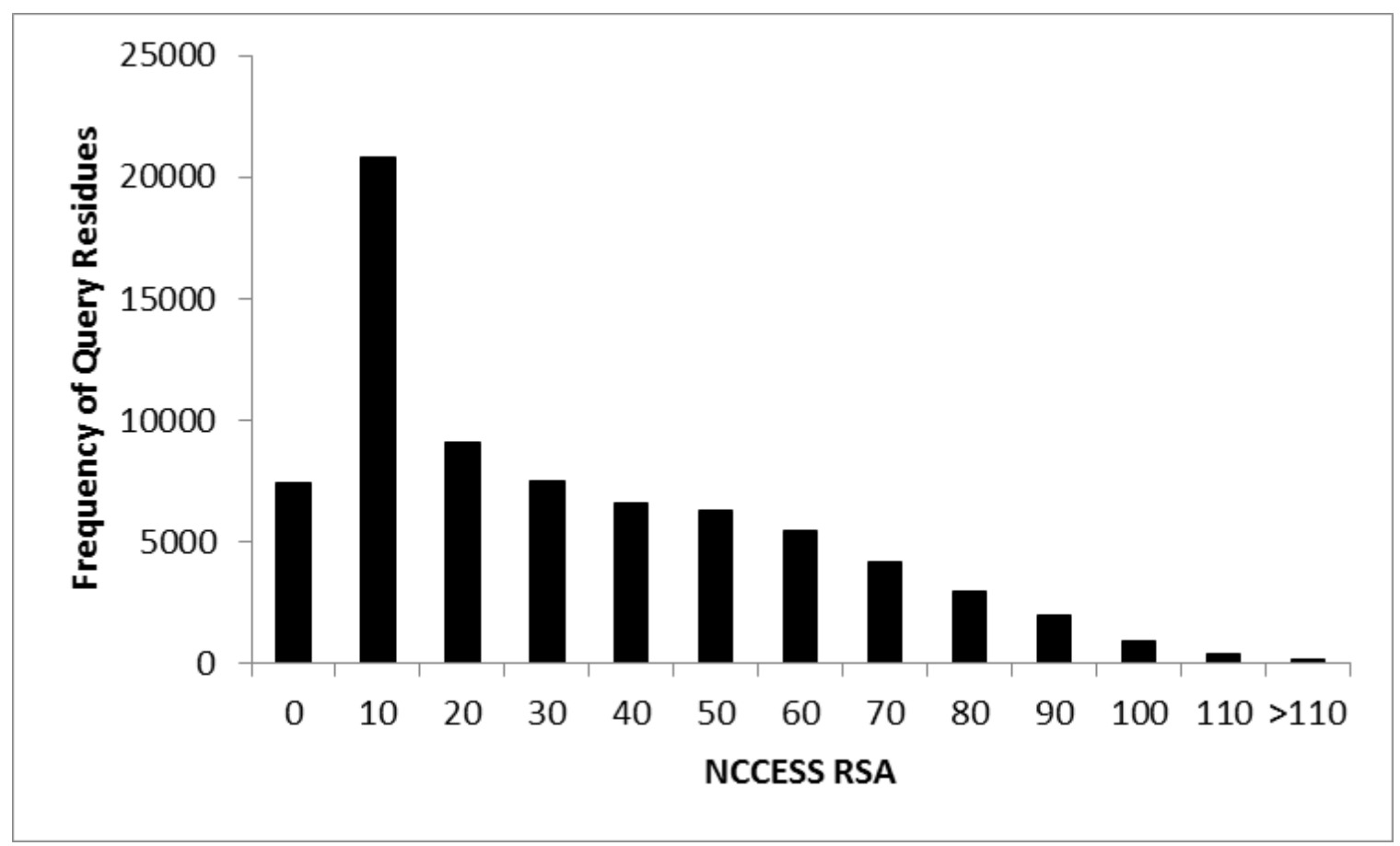

Figure 3.7. Frequency distribution of NACCESS RSA values for various RSA ranges for the 268 training data set. RSA values for a total of 73734 query residues were used for this plot.

Similarly to Figure 3.7, Figure 3.8 shows the frequency distribution of NACCESS RSA values, but for 319,812 residues of the 1363 training data set. In comparison to the 268 NACCESS RSA distribution plot, the 1363 training set also displays a similar trend. The mode of distribution for the 1363 training set also occurs at a NACCESS RSA value of 10 . The 1363 training set also has $38.3 \%$ of residues characterized as buried and $61.7 \%$ of residues on the surface. The mean, mode, and median of this distribution are 27.4, 0, and 19.4, respectively. 


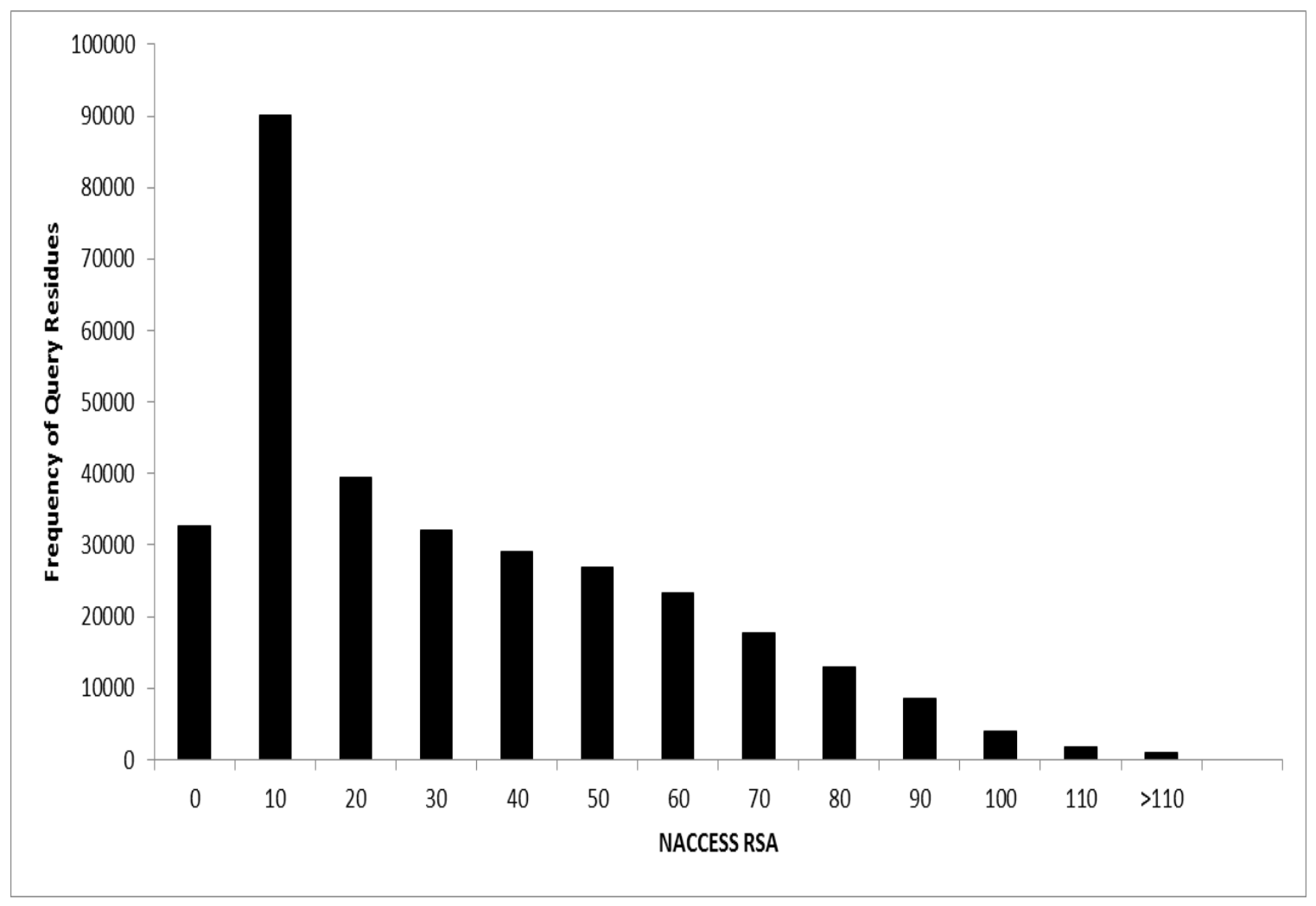

Figure 3.8. Frequency distribution of RSA values for various RSA ranges for the 1363 training data set. RSA values for a total of 319,812 query residues were used for this plot.

Figure 3.9 represents a comparison of RSA distribution of 50,856 residues of the 215 test set for NACCESS RSA and predicted RSA values generated using the 268 training set. The NACCESS RSA value distribution plot is displayed in Figure 3.9. The NACCESS frequency distribution peaks at a NACCESS value at 5, with a total of 9572 residues (18.8\%) displaying this NACCESS value. Both distributions are right-skewed histograms. The NACCESS threshold for buried resides is $\geq 20$, and this distribution indicates about $48.4 \%(24,595)$ of residues can be characterized as buried when derived from X-ray information. The remaining $51.6 \%$ of residues are characterized as appearing on the surface of the protein. The mean, median, and mode of this distribution are 28.5, 
21.6 , and 0 , respectively.

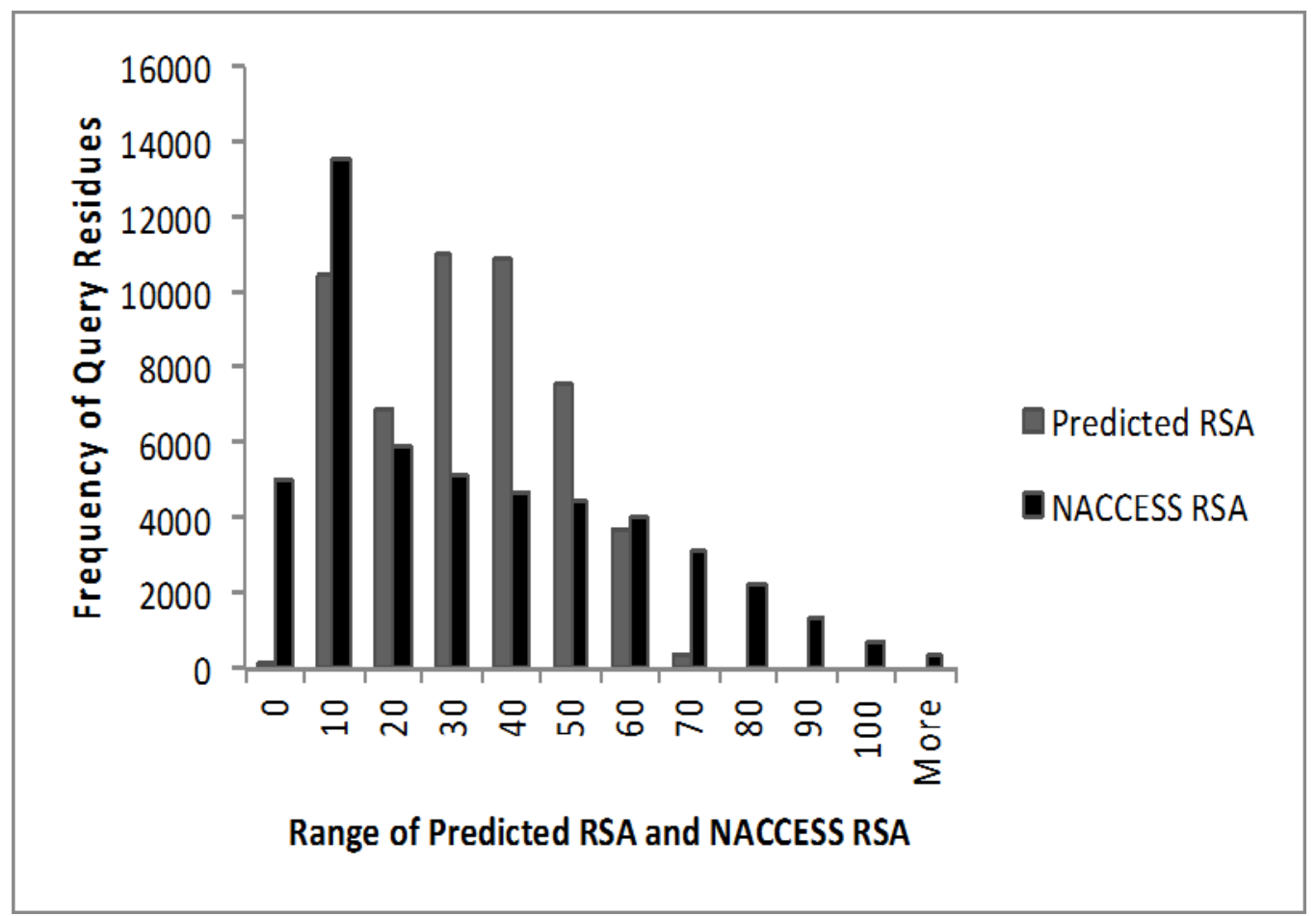

Figure 3.9. Frequency distribution comparison of NACCESS RSA values and predicted RSA values for the 215 test set using the 268 training set. A total of 50,856 residues for a total of 215 protein lists were used to generate these plots. Frequency of query residues with respect to NACCESS RSA values and frequency of query residues with respect to predicted RSA values generated by linear regression are presented.

The second part of Figure 3.9 shows the distribution of predicted RSA values for the 50,856 residues of the 215 test set. Unlike the NACCESS plot, the predicted RSA plot peaks at an RSA value of 10 with a total of $9729(19.1 \%)$, and the second highest peak is observed at a predicted RSA of 35 with 5097 (10.0\%) residues with an RSA of 35. The mean of this distribution is 27.1 , the median is 27.4 , and the mode is 6.4. Unlike NACCESS RSA, where there are 4767 residues (9.4\%) with NACCESS values greater than 70, none of the residues in the predicted RSA are forecasted to have RSA value 
greater than 70. Also, predicted RSA values appear compressed relative to NACCESS RSA.

The comparison of NACCESS RSA and predicted RSA for the 215 test set using 1363 data as a training set is presented in Figure 3.10. Just as in first part of Figure 3.9, Figure 3.10 presents NACCESS RSA distribution for the 50,856 residues of the 215 test set. The second part of Figure 3.10 is the distribution of predicted RSA values for the 215 test set using the 1363 as training set. The highest peak in the predicted RSA generated via using the 1363 as training set is observed at an RSA value of 10. A total of 8693 residues (17.1\%) have predicted RSA values equal to 10 . Similar to the distribution plot of the 215 test set using the 268 as training set (Figure 3.9), very few residues (0.1\%) are predicted to have RSA value equal to 5, the highest corresponding peak in the NACCESS RSA frequency. Again, none of the residues are predicted to have RSA values greater than 70. The mean, median, and mode of this distribution are 28.1, 28.7, and 7.6, respectively. This distribution displays a multimodal distribution pattern. 


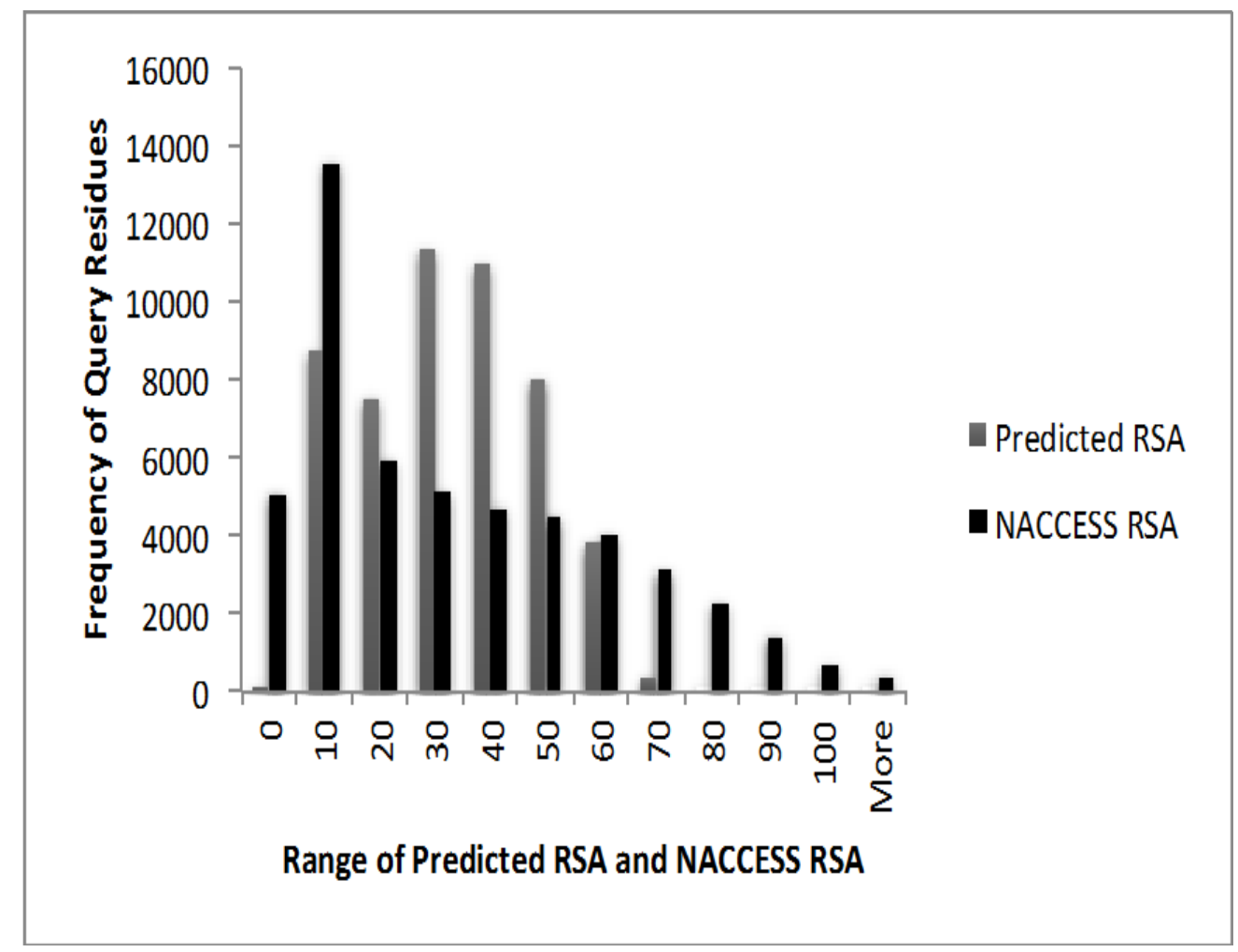

Figure 3.10. Frequency distribution comparison of NACCESS RSA values and predicted RSA values for the 215 test set using the 1363 training set. A total of 50,856 residues for 215 protein lists were used to generate these plots. Frequency of query residues with respect to NACCESS RSA values and frequency of query residues with respect to predicted RSA values generated by linear regression are displayed.

Figure 3.11 presents comparison aggregate correlation plots of sequence entropy and other homology-based parameters for the 268 training data set. Here is the combined aggregate correlation plot for the manually generated BLAST output calculation performed previously by the Lustig group (Mishra, 2010). On the other hand, Figure $3.11 \mathrm{~B}$ is the combined aggregate correlation plot for automatically generated BLAST output calculations. This comparison was made to validate the finding of the automated BLAST output files. Aggregate values for all of the homology-based parameters are determined by averaging their respective values within the same packing density interval. 
The standard deviation for both the manually generated and automatic calculations are comparable, typically 0.3 for E20 and E6, and 0.1 for FSHP and FSR.

Comparable to our previous work (Mishra, 2010; Rose et al., 2011), two major regions were noted in in both the graphs. Major Region I is associated with a packing density of 11 to 25 ( 0.09 to 0.04 of inverse density), and relates to the portion of the graph where average sequence entropy increases linearly with an increase in packing density. On the other hand, in Major Region II, associated with a packing density of 4 to10 ( 0.25 to 0.1 of inverse density), a different trend is observed. In Major Region II, sequence entropy remains almost the same as packing density increases. Both Figures 3.11A and 3.11B show similar trends and patterns. 


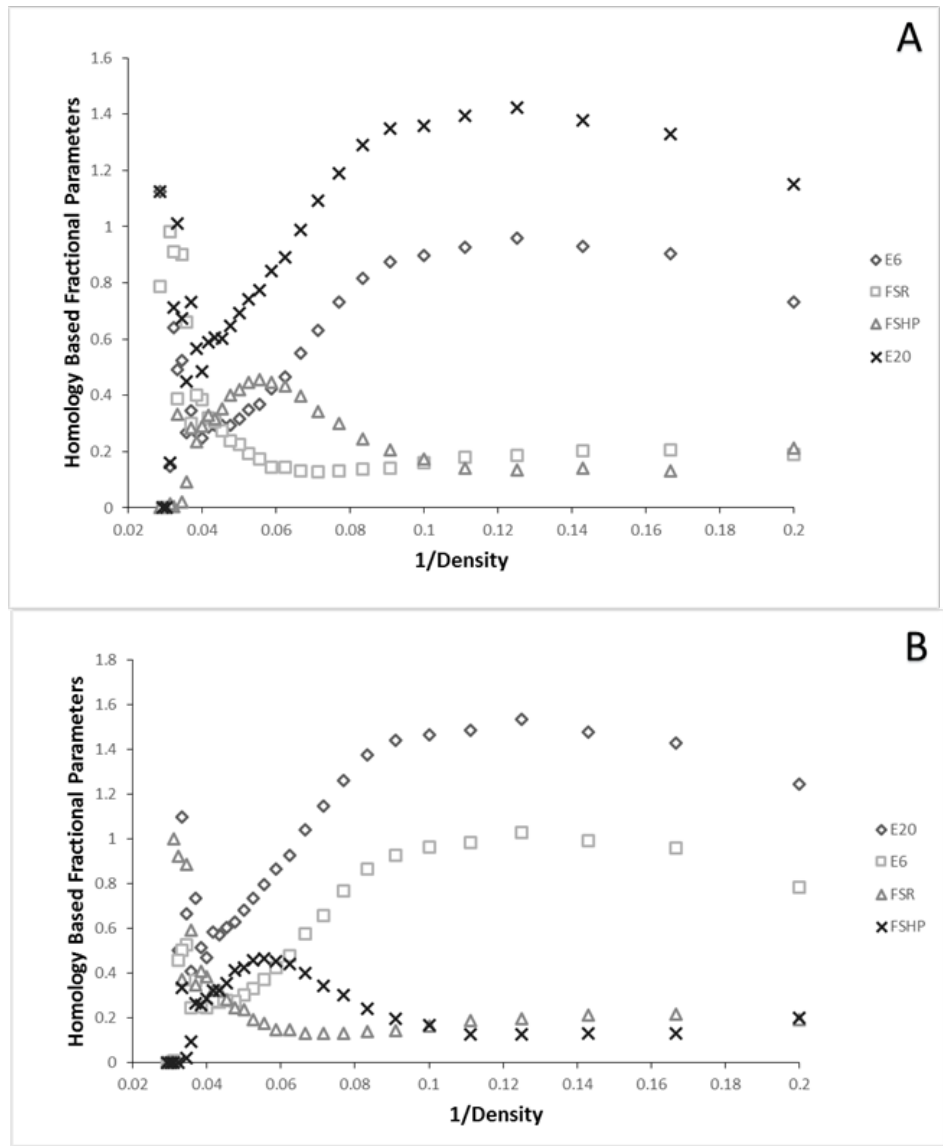

Figure 3.11. Comparison of combined aggregate correlation plots of sequence entropy and other homology-based parameters for the 268 training set. Packing density is the number of $\mathrm{C} \alpha$ within a $9 \AA$ radius, and excluded here is the portion of Region II with packing densities less than 5 ( $<1 \%$ of all residues). Average sequence entropy, E20 (open-square, ordinate) and E6 (closed-diamond), are calculated by averaging the respective values for 73,727 query residues for each inverse packing density value. Fraction of strongly hydrophobic residues (asterisk) and fraction of small residues (opendiamond) are calculated and averaged over a total of 7.12E7 aligned residues, plotted against inverse packing density. Average values for all the homology-based parameters are determined by averaging their respective values within the same packing density interval. Note that the standard deviations for E20 and E6 are comparable (typically 0.3), while typically 0.1 for FSHP and FSR. A. Combined aggregate correlation plot for the manually generated BLAST output calculation (Mishra, 2010). B. Combined aggregate correlation plot for the automatically generated BLAST output calculation. 
Figure 3.12 shows the aggregate correlation plots of sequence entropy and other homology based parameters for the 1363 training data set. The presence of the two major Regions I and II are also observed with comparable trends in the 1363 training set as with the 268 training data sets.

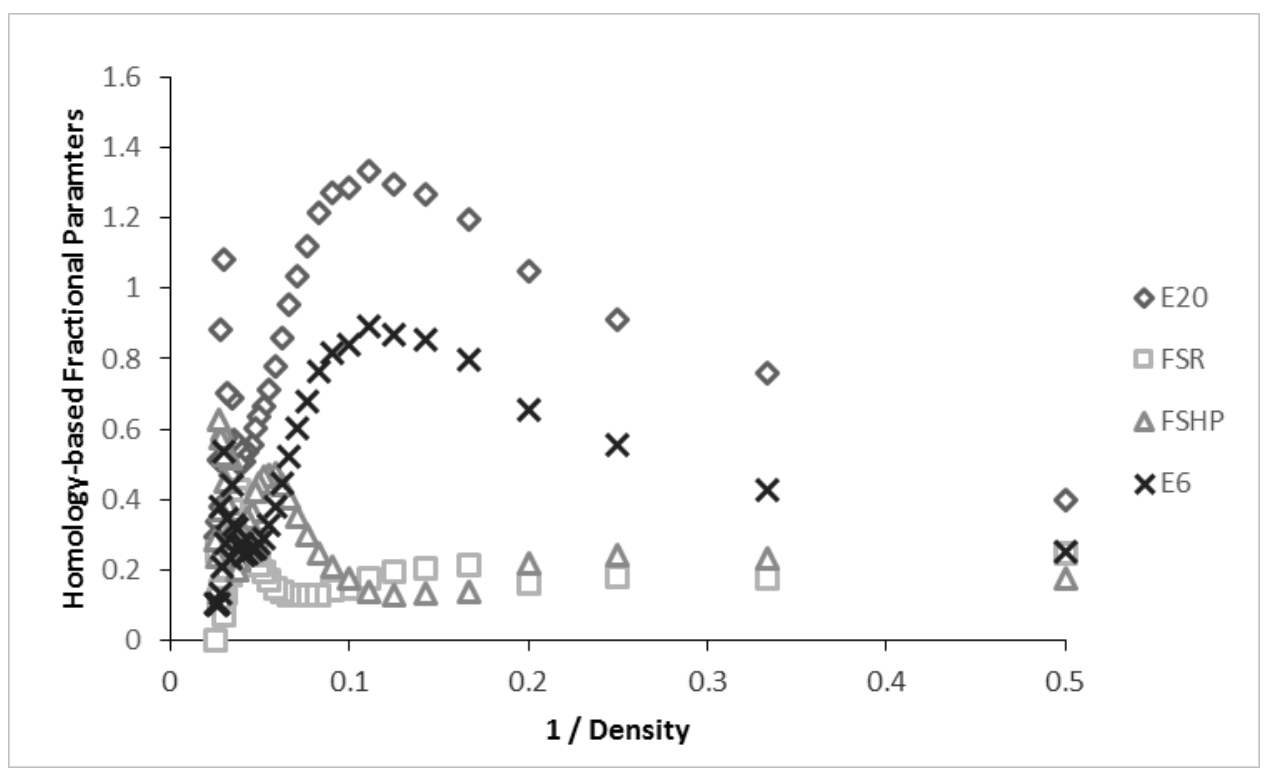

Figure 3.12. Combined aggregate correlation plots of sequence entropy and other homology-based parameters for the 1363 training set. Packing density is the number of C $\alpha$ within a $9 \AA$ radius and that the portion of Region II with a packing density less than 5 is $<1 \%$ of all residues. Average sequence entropy and E6 are calculated by averaging the respective values for 73,734 query residues for each inverse packing density value. Fraction of strongly hydrophobic residues and fraction of small residues are calculated and averaged over a total of 318,840 aligned residues, plotted against inverse packing density. Average values for all the homology-based parameters are determined by averaging their respective values within the same packing density interval. Note that the standard deviations for E20 and E6 are comparable (typically 0.3), while typically 0.1 for FSHP and FSR.

Figures 3.13 and 3.14 represent individual plots of RSA as a function of inverse packing density and packing density for the 268 and 1363 training data sets. Again, 
similar to the aggregate correlation plots of sequence entropy, two major regions are observed with aggregate plots of RSA. In the first major region, RSA values decrease linearly as packing density increases, and in the second major region, RSA values stay almost constant-close to 0 - as packing density increases. Similar trends are observed in both of the training data sets. These concur with our previous findings (Mishra, 2010) that residues associated with lower packing densities have higher RSA and are more likely to be found on the surface of the proteins, whereas residues that have a high packing density are dense and are usually found in the core of the proteins. The RSA values for these residues should be closer to 0 . Figures 3.12 and 3.13 support this claim, as high-density values have RSA close to 0 . Although majority of protein residues display this trend, very few residues have RSA values of 0 for a low packing density. Figures 3.13A and 3.14A simply show this RSA trend as a function of inverse density. 

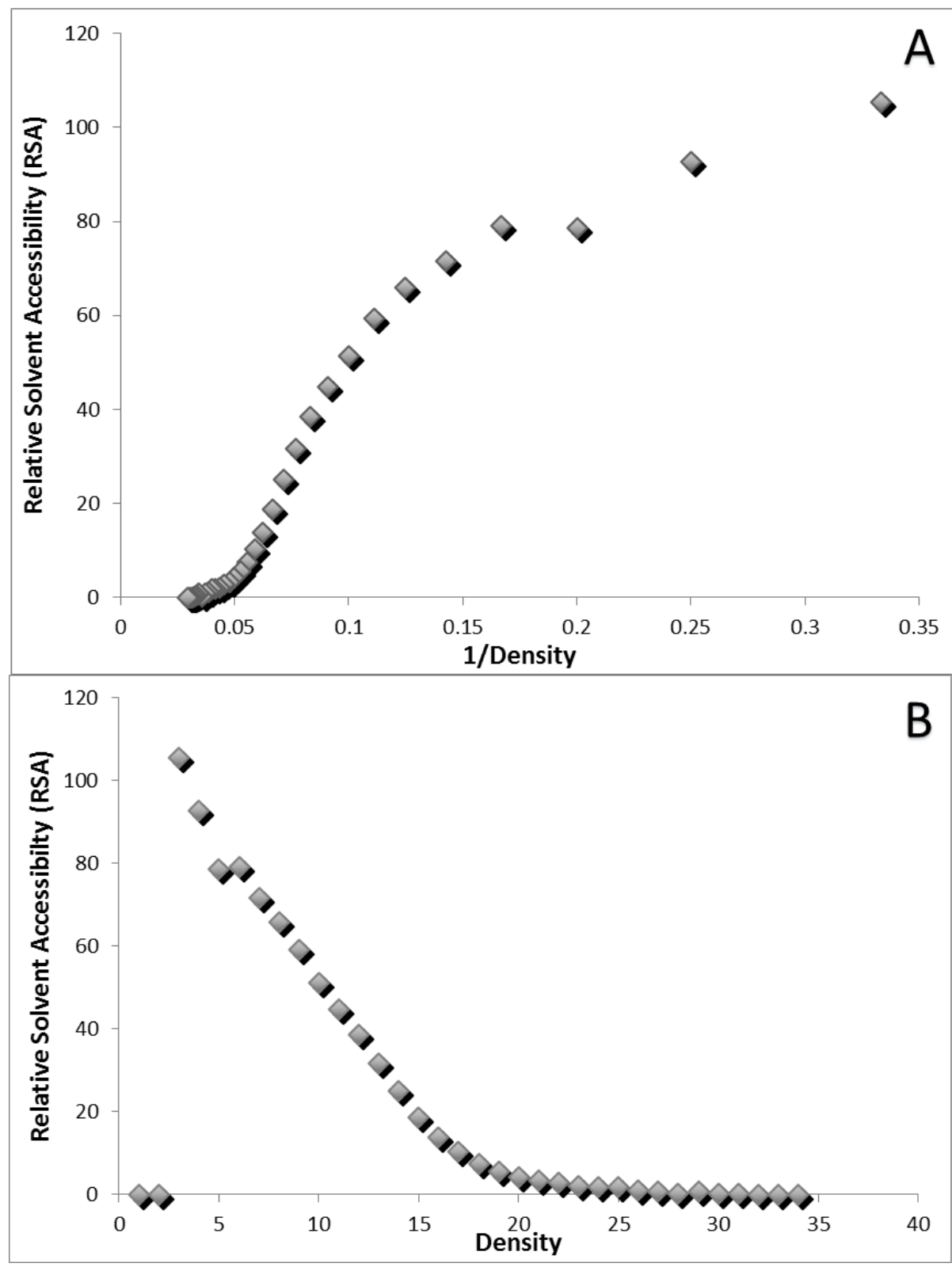

Figure 3.13. Density - relative surface accessibility comparison for the 268 training set. Here the aggregate of RSA values were obtained by averaging a total of 73,734 query residues at each packing density position. A. Aggregate correlation plot of relative surface accessibility (RSA) and inverse packing density for the 268 training set of proteins. B. Aggregate correlation plot of relative surface accessibility (RSA) and packing density for the 268 training set of proteins. 

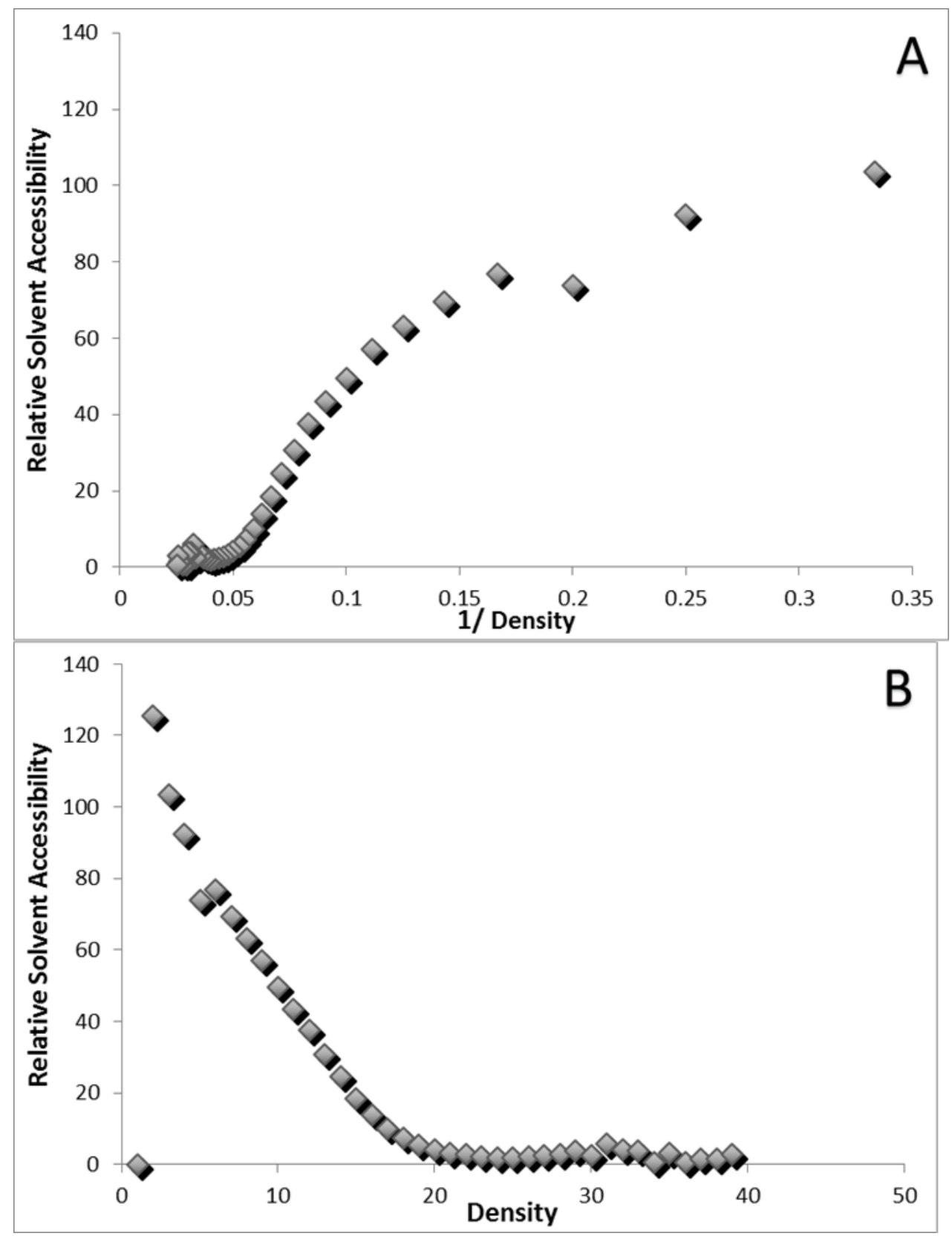

Figure 3.14. Density_relative surface accessibility comparison for the 1363 training set. Here the aggregate of RSA values was obtained by averaging a total of 318,840 query residues at each packing density position. A. Aggregate correlation plot of relative surface accessibility (RSA) and inverse packing density for the 1363 training set of proteins. B. Aggregate correlation plot of relative surface accessibility (RSA) and packing density for the 1363 training set of proteins. 


\subsection{Accuracy of Results}

Table 3.1 showcases a comparison of accuracies for the 12 different linear regression models using the 268 training data set on the 215 test data set for the manually derived BLAST and automated BLAST outputs. The accuracy for the manually derived BLAST output is generally lower than the accuracy of its counterpart model using the automated BLAST output. The accuracies were generated with the same number; 73,734 aligned residues were used for the 268 training data set, and 50,856 aligned residues were used for the 215 test set. Theoretically, these two sets of accuracies should be identical, but due to various parameters involved during the process of accuracy calculation they are not. Previous R scripts (Rose et al., 2011) calculated the manual BLAST output accuracies. First, linear regression for the training data set was generated, and then $\beta$ coefficients that were to be applied to the test set were extracted. The $\beta$ coefficients were also manually fed into the 215 data set RSA prediction calculations. This manual introduction of $\beta$ coefficients into the code presented room for error in calculation. Anytime there was an improvement or modification made to the training set, it altered the $\beta$ coefficients' values. Due to the fact that the entries were made manually, these changes were often not reflected in the application to the test set. The possibility of input truncation was also introduced.

However, in the automated BLAST output accuracies, this sort of error in calculations was eliminated by sequentially generating the linear model using the training data set and applying the parameters obtained from the regression to the test sets automatically and internally in the program. Anytime there were any changes implied in 
the training set, those changes were automatically reflected in the test set. This new automated accuracy code was also implemented in the manual BLAST output calculations, and comparable accuracies with the automated BLAST outputs were observed. The highest accuracy observed was for the E20 + E6 + FSR + FSHP + AA model at $72.4 \%$ and $74.2 \%$, respectively, for the manual and automated version of the 268 training set and the 215 test set. Accuracy of $74.4 \%$ was also observed for the E6 + FSR + FSHP+ AA model. Noteworthy is the fact that the E20 + E6 + AA hovers very close to the highest accuracy at $74 \%$. This also shows that the addition of a qualitative predictor, AA, aided in better accuracy prediction because, without AA, the E20 + E6 model has accuracy of $69.1 \%$, which is lower by $4.9 \%$. Also, the model FSHP + AA is missing from the manual BLAST accuracy because FSHP + AA was incorporated as the $12^{\text {th }}$ model after these values were generated. 
Table 3.1. Comparison of regression accuracy using manually generated BLAST output calculation and automatically generated BLAST output calculations.

\begin{tabular}{|l|l|l|}
\hline Models & \multicolumn{2}{|c|}{ Accuracy } \\
\hline & $\begin{array}{l}\text { Manual 268 } \\
\text { Training } \\
215 \text { Test }\end{array}$ & $\begin{array}{l}\text { Automated 268 Training } \\
215 \text { Test }\end{array}$ \\
\hline E20 & 63.1 & 63.0 \\
\hline E6 & 67.0 & 68.9 \\
\hline FSHP & 67.3 & 68.2 \\
\hline FSHP + AA & & 69.8 \\
\hline AA & 70.6 & 70.4 \\
\hline E20 + AA & 72.1 & 72.8 \\
\hline E6 + AA & 73.1 & 74.0 \\
\hline E20 + E6+ AA & 72.9 & 74.0 \\
\hline E20 + E6 & 67.4 & 69.1 \\
\hline $\begin{array}{l}\text { E20 + FSR + FSHP + } \\
\text { AA }\end{array}$ & 73.4 & 73.3 \\
\hline $\begin{array}{l}\text { E6 + FSR + FSHP + } \\
\text { AA }\end{array}$ & 73.2 & 74.2 \\
\hline $\begin{array}{l}\text { E20 + E6 + FSR + } \\
\text { FSHP + AA }\end{array}$ & 72.4 & 74.2 \\
\hline
\end{tabular}

Table 3.2 displays a summary of regression accuracies for 12 models tested for the 215 test set using 268 as the training set, the 215 test set using 1363 as the training set, and finally the 215 PSI-BLAST test set using 268 PSI-BLAST as the training set. This set of 12 models accuracy calculations is also referred to as standard accuracy calculation for the remainder of this thesis. The NACCESS RSA threshold used for the 268 training set, and the 268 PSI-BLAST training set was $>23$, while $>25.2$ was used for the 1363 training set. These thresholds were determined with the aid of linear regression line of best fit (Figures 2.2 and 2.3) for each of the training sets.

Here again as in Table 3.1, we observe similar trends. Consistently, for all of the training and test set combinations used, E20 + E6 + FSR + FSHP + AA has the highest prediction accuracy observed. Our findings here indicate that, although the highest 
accuracy for the PSI-BLAST model does slightly better, at $74.4 \%$ vs. $74.2 \%$ for regular BLAST, it does not have a significantly large impact. Also for some models, like E6 + FSR + FSHP + AA, PSI-BLAST actually results in lower accuracy than BLAST at $69.09 \%$ vs. $73.29 \%$. As was seen in Table 3.1, the addition of the qualitative predictor AA improves accuracy.

Table 3.2. Summary of regression accuracy for the 12 models tested. The three different sets of numbers represent different training and test set models. The predicted RSA thresholds for the three sets were slightly different depending on the non-hydrophobic linear regression intercept for each data set.

\begin{tabular}{|c|c|c|c|}
\hline Models & \multicolumn{3}{|c|}{ Accuracy } \\
\hline & $\begin{array}{l}268 \text { Training }^{1} \\
215 \text { Test }^{1}\end{array}$ & $\begin{array}{l}1363 \text { Training }^{2} \\
215 \text { Test }^{2}\end{array}$ & $\begin{array}{l}268 \text { PSI-BLAST Training } \\
215 \text { PSI-BLAST Test }\end{array}$ \\
\hline E20 & 63.0 & 63.3 & 63.1 \\
\hline E6 & 68.9 & 68.8 & 68.8 \\
\hline FSHP & 68.3 & 68.2 & 68.2 \\
\hline FSHP + AA & 69.7 & 70.8 & 70.8 \\
\hline AA & 70.4 & 70.4 & 70.4 \\
\hline $\mathrm{E} 20+\mathrm{AA}$ & 72.8 & 72.9 & 72.9 \\
\hline $\mathrm{E} 6+\mathrm{AA}$ & 74.0 & 74.1 & 74.1 \\
\hline $\mathrm{E} 20+\mathrm{E} 6+\mathrm{AA}$ & 74.0 & 74.1 & 74.1 \\
\hline E20 + E6 & 69.1 & 69.1 & 74.1 \\
\hline $\begin{array}{l}\mathrm{E} 20+\mathrm{FSR}+\mathrm{FSHP}+ \\
\mathrm{AA}\end{array}$ & 73.3 & 74.1 & 69.1 \\
\hline $\begin{array}{l}\mathrm{E} 6+\mathrm{FSR}+\mathrm{FSHP}+ \\
\mathrm{AA}\end{array}$ & 74.2 & 74.4 & 74.1 \\
\hline $\begin{array}{l}\mathrm{E} 20+\mathrm{E} 6+\mathrm{FSR}+ \\
\mathrm{FSHP}+\mathrm{AA}\end{array}$ & 74.2 & 74.4 & 74.4 \\
\hline
\end{tabular}

${ }^{1}$ Predicted RSA threshold used $>23$.

${ }^{2}$ Predicted RSA threshold used $>25.2$.

${ }^{3}$ Predicted RSA threshold used $>23$. 


\subsection{Outcome of Additional Methods to Improve Prediction Accuracy}

\subsubsection{Outcome of Additional Models Applied}

Separate predictors for each of the small residues Ala and Gly are worth noting here. In our previous models, Ala and Gly were incorporated under one sequence homology parameter, FSR. The fraction of aligned residues that are Gly (FG) and Ala (FA) were then generated. New models that substituted FSR with FA, FG, and FA + FG were generated. The accuracies for each of these additional models are presented in Table 3.3. The highest accuracy observed as a result of the incorporation of these additional models was $73.6 \%$ and $73.4 \%$ for the 268 and 1363 training data sets, respectively. Contrary to our hypothesis, separating the Ala and Gly from the existing FSR sequence homology did not improve the overall prediction accuracy. Interestingly for both the 268 and 1363 training sets, the accuracies with the 215 set hovered between $73.0 \%$ and $73.6 \%$. While we observed variant accuracy ranges for all of the other regression models we applied (see Tables 3.1 and 3.2), it is noteworthy that the range of accuracies involving FG and FA did not improve over the standard methods. 
Table 3.3. Regression accuracy table for additional models applied. The two different sets of numbers represent different training and test set models. The predicted RSA thresholds for the two sets were slightly different depending on the non-hydrophobic linear regression intercept for each data set. $>23$ and $>25.2$ were used, respectively, for the 268 BLAST-215 BLAST and1363 BLAST-215 BLAST.

\begin{tabular}{|l|l|l|}
\hline \multicolumn{1}{|c|}{ Models } & \multicolumn{2}{c|}{ Accuracy } \\
\hline & $\begin{array}{l}\text { 268 Training } \\
215 \text { Test }\end{array}$ & $\begin{array}{l}1363 \text { Training } \\
215 \text { Test }\end{array}$ \\
\hline FSHP + AA & 69.7 & 70.8 \\
\hline E20 + FA + FG + FSHP + AA & 73.3 & 73.0 \\
\hline E20 + FA + FSHP + AA & 73.3 & 73.0 \\
\hline E20 + FG + FSHP + AA & 73.2 & 73.0 \\
\hline E6 + FA + FG + FSHP + AA & 73.6 & 73.4 \\
\hline E6 + FA + FSHP + AA & 73.6 & 73.4 \\
\hline E6 + FG + FSHP + AA & 73.4 & 73.4 \\
\hline $\begin{array}{l}\text { E20 + E6 + FA + FG + FSHP + } \\
\text { AA }\end{array}$ & 73.6 & 73.4 \\
\hline E20 + E6 + FA + FSHP + AA & 73.6 & 73.4 \\
\hline E20 + E6 + FA + FSHP + AA & 73.5 & 73.4 \\
\hline
\end{tabular}

\subsubsection{Outcome of Categorized Protein Data Set}

In the Hotpatch study by Petit and coworkers (2007), a total of 618 PDB IDs are included. Out of the two categories investigated to explore the possibility of accuracy improvement, only the protein binding group yielded better accuracy. Table 3.4 displays protein binding PDB ID matches between the 618 set and each of the two training data sets (268 and 1363) and the test set. There were 13 PDB ID matches between the 618 and 268 training sets, 8 PDB ID matches between the 618 and 1363 training sets, and a total of 16 PDB ID matches between the 618 and 215 test sets for the protein-binding category. 
Table 3.4. Protein binding category PDB ID matches between the 618 Hotpatch protein PDB IDs (Petit et al., 2007) and the 268 and 1363 training sets and 215 test set.

\begin{tabular}{|c|c|c|c|}
\hline & \multicolumn{3}{|c|}{ Protein Binding PDB ID Matches } \\
\hline & 618-268 matches & 618-1363 matches & 618-215 matches \\
\hline 1. & 1AK4C & 1BKRA & 1BEOA \\
\hline 2. & 1B3AA & 1G3PA & 1BGCA \\
\hline 3. & 1BUOA & 1KPTA & 1CFYA \\
\hline 4. & 1FINB & 1KWAA & 1CSGA \\
\hline 5. & 1KPTA & 1TENA & 1JKWA \\
\hline 6. & 1KWAA & 1VCAA & $1 \mathrm{KNBA}$ \\
\hline 7. & 1M6PA & 2PSPA & 1KPTA \\
\hline 8. & $10 S P O$ & 3SEBA & 1LKIA \\
\hline 9. & 1YCSA & & 1LKKA \\
\hline 10. & 1YCSB & & 1MAZA \\
\hline 11. & 2ILKA & & $10 S P O$ \\
\hline 12. & 2TGIA & & 1SIGA \\
\hline 13. & 2TRCP & & 1SVPA \\
\hline 14. & & & 1VCAA \\
\hline 15. & & & 1WHIA \\
\hline 16. & & & 2PSPA \\
\hline
\end{tabular}

There were two sets of regressions carried out for the protein binding categories. For the first set of regression analyses, 618 -268 protein binding (PB) matches were used as the training set and 618-215 protein binding matches were used as the test set. For the second set of regression analyses, 618-1363 protein binding matches were used as the training set and 618-215 protein binding matches were used as the test set. The prediction accuracy for the 12 different models is presented in Table 3.5.

As observed from the accuracy table (see Table 3.5), the PB category yielded slightly better prediction accuracy than our previous regression analysis (see Tables 3.1, 3.2, and 3.4). In standard calculation, the highest accuracy achieved was $74.4 \%$ for the 1363 training set and the 215 test set calculations. The highest accuracy value for the PB 
is at $76.3 \%$ for $618-268 \mathrm{~PB}$ matches as training set and $618-215 \mathrm{~PB}$ matches for the test set. Both E20 + E6 + FSR + FSHP + AA and E6 + FSR + FSHP + AA display the highest predicted accuracy for this regression analysis at 76.0. Both sets of regressions display similar patterns and accuracy amounts.

Table 3.5. Regression accuracy table for protein binding model. The two different sets of numbers represent different training and test set models. The predicted RSA thresholds for the two sets were $>23$ and $>25.2$, respectively, for the 618-268 and 1363268 matches. Here, PB stands for protein-binding.

\begin{tabular}{|l|c|c|}
\hline Models & $\begin{array}{l}\text { Training =618-268 PB } \\
\text { PDB IDs Matches } \\
\text { Test=618-215 PB PDB } \\
\text { IDs Matches }\end{array}$ & $\begin{array}{l}\text { Training=618-1363 PB } \\
\text { PDB IDs Matches } \\
\text { Test=618-215 PB PDB } \\
\text { IDs Matches }\end{array}$ \\
\hline E20 & 60.5 & 60.5 \\
\hline E6 & 60.5 & 60.5 \\
\hline FSHP & 73.0 & 74.3 \\
\hline FSHP + AA & 74.2 & 75.8 \\
\hline AA & 73.0 & 73.4 \\
\hline E20 + AA & 74.8 & 74.5 \\
\hline E6 + AA & 75.4 & 75.4 \\
\hline E20 + E6 + AA & 75.0 & 75.5 \\
\hline E20 + E6 & 60.1 & 61.4 \\
\hline E20 + FSR + FSHP + AA & 76.0 & 75.3 \\
\hline E6 + FSR + FSHP + AA & 76.2 & 76.0 \\
\hline E20 + E6 + FSR + FSHP + & 76.3 & 76.0 \\
AA & & \\
\hline
\end{tabular}

${ }^{1}$ The predicted RSA threshold used for this set of calculations was $>23$.

${ }^{2}$ The predicted RSA threshold used for this set of calculations was $>25.2$.

\subsubsection{Use of All 618 PDB IDs as Training and 215 as Test}

Another test performed on the 618 set was the use of all 618 proteins as the training set and the 215 as the test set. There were four different sets of linear regression calculations involving the 618 and 215 protein sets: 1.generic, 2. protein binding, 
3. Oligomers, 4. generic without PB and Oligomers. Table 3.6 includes a comprehensive presentation of the actual application of these sets of calculations.

Table 3.6. Description of 618 PDB IDs as training and 215 PDB IDs as test regression analysis calculations. This table summarizes the different groups of PDB IDs used as training and test sets for this analysis.

\begin{tabular}{|l|l|l|}
\hline 1) Generic & Training & Test \\
\hline 2) Protein Binding (PB) & All of the 618 protein & $\begin{array}{l}\text { All the PDBs in the 215 set } \\
\text { that match } 618 \text { set }\end{array}$ \\
\hline 3) Oligomers & $\begin{array}{l}\text { Oligomer PDB IDs from } \\
\text { the } 618^{2}\end{array}$ & $\begin{array}{l}\text { Oligomer PDBs matches } \\
\text { the } 618 \text { and } 215 \text { sets } \\
\text { between the } 618 \text { and } 215 \\
\text { sets }\end{array}$ \\
\hline 4) $\begin{array}{l}\text { Generic without PB } \\
\text { and Oligomers }\end{array}$ & $\begin{array}{l}618 \text { PDBs excluding PB } \\
\text { and oligomers }^{3}\end{array}$ & $\begin{array}{l}\text { All } 618-215 \text { matches } \\
\text { without PB and oligomers }\end{array}$ \\
\hline
\end{tabular}

${ }^{1} 618-215$ PB matches were excluded.

${ }^{2} 618-215$ Oligomer matches were excluded.

${ }^{3} 618-215$ matches were excluded.

Table 3.7 is the regression accuracy results of the different set calculations presented in Table 3.6. Out of the four categories, protein binding consistently had higher accuracies for each of 12 models tested, while oligomers reliably had lower accuracies for each model. Protein binding model, RSA $=$ E20 + FSR + FSHP + AA, had the utmost accuracy, $77.7 \%$, of all the models for the various categories tested. The addition of the amino acid (AA) qualitative predictors to each of the homology-based parameters used as models generally resulted in higher accuracies compared to the same model without an AA qualitative predictor. 
Table 3.7. Regression accuracy table of PDB IDs of the four groups: 1. generic 2. protein binding 3. oligomers 4. and generic without PB and Oligomers used in the regression analysis. Here the entire categorized 618-protein sets were used as training, and

\begin{tabular}{|l|l|l|l|l|}
\hline \multicolumn{1}{|c|}{ Model } & Generic & $\begin{array}{l}\text { Protein } \\
\text { Binding (PB) }\end{array}$ & Oligomer & $\begin{array}{l}\text { Generic } \\
\text { without PB and } \\
\text { Oligomer }\end{array}$ \\
\hline RSA = E20 & 62.1 & 60.5 & 61.0 & 63.3 \\
\hline RSA = E6 & 68.8 & 60.5 & 68.2 & 70.1 \\
\hline RSA = FSHP & 69.8 & 74.0 & 68.3 & 71.9 \\
\hline $\begin{array}{l}\text { RSA }=\text { FSHP } \\
+ \text { AA }\end{array}$ & 71.0 & 75.8 & 69.8 & 72.3 \\
\hline RSA = AA & 71.2 & 74.4 & 70.3 & 70.9 \\
\hline $\begin{array}{l}\text { RSA }=\text { E20 + } \\
\text { AA }\end{array}$ & 73.5 & 76.0 & 72.3 & 74.6 \\
\hline $\begin{array}{l}\text { RSA }=\text { E6 + } \\
\text { AA }\end{array}$ & 74.6 & 76.9 & 73.1 & 75.1 \\
\hline $\begin{array}{l}\text { RSA = E20 + } \\
\text { E6 + AA }\end{array}$ & 74.5 & 76.7 & 73.2 & 75.2 \\
\hline $\begin{array}{l}\text { RSA = E20 + } \\
\text { E6 }\end{array}$ & 69.1 & 63.1 & 68.7 & 70.3 \\
\hline $\begin{array}{l}\text { RSA = E20 + } \\
\text { FSR + FSHP } \\
+ \text { AA }\end{array}$ & 74.0 & 76.9 & 72.7 & 75.1 \\
\hline $\begin{array}{l}\text { RSA = E6+ } \\
\text { FSR + FSHP } \\
+ \text { AA }\end{array}$ & 74.7 & 77.2 & 73.5 & 75.3 \\
\hline $\begin{array}{l}\text { RSA = E20+ } \\
\text { E6 + FSR + } \\
\text { FSHP + AA }\end{array}$ & 74.7 & 77.3 & 73.4 & 75.4 \\
\hline
\end{tabular}

categorized PDB IDs from the 215 were used as test set.

\subsection{Incorporation of the Categories to the Existing Data Sets}

There are several PDB IDs that match PDB IDs in the 268, 215, and 1363 data sets. The last set of regression analyses took advantage of the PDB IDs that overlap between the different data sets with 618 . Similar to the previous set of calculations with 
the 618 data set, these regression calculations were also calculated for four categories of PDB ID: 1. Generic, 2. protein binding (PB), 3. Oligomers, 4. generic without PB and oligomers.

As the first task, all the PDB IDs that were common between the 618 and 215 data set, 618 and 268 data set, and 618 and 1363 data set were extracted for each of the categories. The protein binding category had a total of 17 PDB IDs in 215, 12 in 268, and 8 in 1363 common with the 618 protein binding category. The oligomer category shared 30 PDB IDs in 215, 25 in 268, and 12 in 1363 with 618 in the oligomer category. Generic PDB IDs are all of the PDB IDs in the various categories in the 618 data set. Generic matches between each of the data sets simply are the number of PDB IDs common between each of the data sets with the 618 data set. All PDB ID matches between the 618 data set and each of the three data sets $(268,215$, and 1363) are presented in Tables 3.8 through 3.10 .

Tables 3.11 through 3.14 are the regression accuracies for the different categories using PDB IDs that are common between the 618 data set and each of the three original data sets $(268,1363$, and 215$)$. Table 3.11 is the regression accuracy for the generic category. Generic PDBs are the PDBs that were found in common between the 618 data set and the 268,215, and 1363 group for all of the categories listed in the 618 data set. The highest accuracy for the generic category was achieved at $74.8 \%$ for the RSA $=$ E20 + E6 + FSR + FSHP + AA model using all of the 618-268 common PDBs as the training set and all of the 618-215 common PDBs as the test set. 
The regression accuracies for the 12 models for the protein binding category are presented in presented in Table 3.12. The highest accuracy for the protein binding category is $76.9 \%$ for the RSA $=\mathrm{E} 20+\mathrm{E} 6+\mathrm{FSR}+\mathrm{FSHP}+\mathrm{AA}$ model using protein binding from 1363 as the training set and protein binding from 215 as the test set. Similar to earlier calculations, the protein binding category yielded the overall highest prediction accuracies. 
Table 3.8. PDB ID matches between the 618 data set with three data sets $(215,268$, and 1363 ) for the protein binding and oligomer categories. Note: A PDB ID categorized as protein binding can also be categorized as oligomer.

\begin{tabular}{|c|c|c|c|c|c|}
\hline \multicolumn{3}{|c|}{ Protein Binding } & \multicolumn{3}{|c|}{ Oligomers } \\
\hline 215 & 268 & 1363 & 215 & 268 & 1363 \\
\hline $1 \mathrm{BEOA}$ & $1 Y C S B$ & 1BKRA & 1QAPA & 1KPFA & 2PSPA \\
\hline $1 \mathrm{BGCA}$ & $10 \mathrm{SPO}$ & $2 \mathrm{PSPA}^{1}$ & 1DOSA & 3CLAA & $1 \mathrm{~A} 73 \mathrm{~A}$ \\
\hline 1CFYA & 1AK4C & 3SEBA & $1 \mathrm{HGXA}$ & 1AJSA & 1HFES \\
\hline $1 \mathrm{CSGA}$ & 1BUOA & 1KPTA & $2 \mathrm{PSPA}^{1}$ & $2 \mathrm{SICl}$ & 1UTGA \\
\hline 1DKTB & 1B3AA & 1TENA & 1 SVPA $^{1}$ & 1BD0A & 1MTYB \\
\hline 1JKWA & $2 \mathrm{TGIA}$ & 1KWAA & 1BBPA & $1 B \cup O A$ & 1B5EA \\
\hline $1 \mathrm{KNBA}^{1}$ & 1KPTA & IVCAA & 1TFEA & 1VLBA & $3 \mathrm{CHBD}$ \\
\hline 1KPTA & 1FINB & 1G3PA & 1BTMA & 1AORA & 1PSRA \\
\hline $1 \mathrm{LKIA}$ & $1 \mathrm{M} 6 \mathrm{PA}$ & & $1 \mathrm{GSAA}$ & 1B3AA & 1BEBA \\
\hline 1LKKA & 1KWAA & & 1KNYA & 1UTGA & 1REGX \\
\hline 1MAZA & 2ILKA & & $3 \mathrm{SDHA}$ & $2 \mathrm{TGIA}$ & $2 \mathrm{SQCA}$ \\
\hline $10 S P O$ & 2TRCP & & $1 \mathrm{ABRB}$ & $3 \mathrm{SDHA}$ & 10TFA \\
\hline 1SIGA & & & 1IDAA & 1B5EA & \\
\hline $1 S V P A^{1}$ & & & $1 E C P A$ & 1GOTB & \\
\hline 1VCAA & & & 1DELA & 1GVPA & \\
\hline $1 \mathrm{WHIA}$ & & & $3 \mathrm{MINB}$ & $1 \mathrm{CG} 2 \mathrm{~A}$ & \\
\hline $2 \mathrm{PSPA}^{1}$ & & & 1GOTB & 1NOXA & \\
\hline & & & 1PDOA & $1 \mathrm{M} 6 \mathrm{PA}$ & \\
\hline & & & 1DKZA & 3DAPA & \\
\hline & & & $1 \mathrm{NOXA}$ & 2ILKA & \\
\hline & & & 1PEAA & 12ASA & \\
\hline & & & 2TYSA & 1REGX & \\
\hline & & & 1FDSA & 1HJRA & \\
\hline & & & 1XVAA & 1DPGA & \\
\hline & & & 1AFRA & $2 S Q C A$ & \\
\hline & & & 1HSBA & & \\
\hline & & & 1OFGA & & \\
\hline & & & 1YASA & & \\
\hline & & & 1DHRA & & \\
\hline & & & $1 \mathrm{KNBA}^{1}$ & & \\
\hline
\end{tabular}

\footnotetext{
${ }^{1}$ Represents PDB IDs that is common between the protein binding category and Oligomer Category.
} 
Table 3.9. PDB ID matches between the 618 data set with three data sets $(215,268$, 1363) between all categories.

\begin{tabular}{|c|c|c|c|c|c|c|}
\hline \multicolumn{7}{|c|}{ Generic } \\
\hline \multicolumn{3}{|c|}{215} & \multicolumn{2}{|c|}{268} & \multicolumn{2}{|c|}{1363} \\
\hline 1LKKA & 3SDHA & 1ABRB & 1YCSB & 1KWAA & 1BKRA & 2PIAA \\
\hline 1MAIA & $5 P 21 A$ & 1AFRA & 1KPFA & 1AYLA & 1XFFA & 1REGX \\
\hline 1MAZA & 1KTEA & 1AXNA & 3 CLAA & 1THTA & 1PINA & 1G3PA \\
\hline 1NOXA & 1LBAA & 1BBPA & 1FJMA & 2ILKA & 2PSPA & 2SQCA \\
\hline 10FGA & 1LCLA & 1BEOA & $10 S P O$ & 12ASA & $2 \mathrm{SCPA}$ & 10TFA \\
\hline $10 S P O$ & 1LKIA & $1 \mathrm{BGCA}$ & $1 \mathrm{AK} 4 \mathrm{C}$ & 2TRCP & 256BA & \\
\hline 1PDOA & 2PSPA & 1BIBA & 2SCPA & 1REGX & 1A73A & \\
\hline 1PEAA & 2RN2A & 1BTMA & 1AJSA & 1NO3A & $1 \mathrm{XXHA}$ & \\
\hline 1POCA & 2SCPA & 1BTNA & $2 \mathrm{SICl}$ & 1HJRA & 3SEBA & \\
\hline 1POTA & 2TYSA & 1CFYA & $1 \mathrm{TX} 4 \mathrm{~A}$ & 1DPGA & 1UTGA & \\
\hline 1QAPA & $3 \mathrm{CHYA}$ & 1CHDA & 256BA & 1STFI & 2FDNA & \\
\hline 1RCFA & 3MINB & 1CNVA & 1NP4A & 2SQCA & $1 \mathrm{~A} 62 \mathrm{~A}$ & \\
\hline 1RECA & 1IDAA & 1CSGA & 2LIVA & 2MBRA & 1MTYB & \\
\hline 1RSYA & 1IDOA & 1DELA & 1BD0A & $1 \mathrm{~A} 48 \mathrm{~A}$ & 1B5EA & \\
\hline 1SBPA & 1JKWA & 1DHRA & 1BUOA & 1A6QA & 1KРTA & \\
\hline 1SIGA & 1KNBA & 1DKTB & 1VLBA & 1GVPA & 1AMUA & \\
\hline 1SMEA & 1KNYA & 1DKZA & $1 \mathrm{HIAI}$ & 2RN2A & 1MPGA & \\
\hline 1SRAA & 1КРТА & 1DOSA & 1AORA & 1CG2A & $1 I I B A$ & \\
\hline 1STFI & & 1ECEA & 1BLZA & 1NOXA & 1FDRA & \\
\hline 1SVPA & & 1ECPA & $4 \mathrm{HTCl}$ & 1FINB & $3 \mathrm{CHBD}$ & \\
\hline 1TFRA & & 1EXNB & 1B3AA & 1M6PA & $1 \mathrm{NBCA}$ & \\
\hline 1VCAA & & 1FDSA & 1UTGA & 3DAPA & 1CIPA & \\
\hline 1WHIA & & 1FJMA & 2TGIA & 2TPSA & 1TENA & \\
\hline 1XVAA & & 1FTPA & 1AKOA & & 2TPSA & \\
\hline 1YASA & & 1GAIA & $3 \mathrm{SDHA}$ & & 1KWAA & \\
\hline 256BA & & 1XFFA & 1B5EA & & 1JFRA & \\
\hline 2AYHA & & $1 \mathrm{GPCA}$ & 1AH7A & & 1PSRA & \\
\hline 2GDMA & & 1GSAA & 13PKA & & 1VCAA & \\
\hline 2LIVA & & 1HGXA & 1KРTA & & 1A8LA & \\
\hline 2MTAC & & 1HLBA & 1AMUA & & 1BEBA & \\
\hline 2PIAA & & $1 \mathrm{HSBA}$ & 1MPGA & & 2GDMA & \\
\hline
\end{tabular}


Table 3.10. PDB ID matches between the 618 data set and the three data sets $(215,268$, and 1363) between all categories without the protein binding and oligomer matches.

\begin{tabular}{|c|c|c|c|}
\hline \multicolumn{4}{|c|}{ Generic without Protein Binding and Oligomers } \\
\hline \multicolumn{2}{|c|}{215} & 268 & 1363 \\
\hline 1AXNA & 1SBPA & 1FJMA & \\
\hline 1BIBA & 1SMEA & 2SCPA & 1XFFA \\
\hline 1BTNA & 1SRAA & 1TX4A & 1PINA \\
\hline 1CHDA & 1STFI & 256BA & 2SCPA \\
\hline 1CNVA & 1TFRA & 1NP4A & 256BA \\
\hline 1ECEA & 256BA & 2LIVA & $1 \mathrm{XXHA}$ \\
\hline 1EXNB & 2AYHA & $1 \mathrm{HIAl}$ & 2FDNA \\
\hline 1FJMA & 2GDMA & 1BLZA & $1 \mathrm{~A} 62 \mathrm{~A}$ \\
\hline 1FTPA & 2LIVA & $4 \mathrm{HTCl}$ & 1AMUA \\
\hline 1GAIA & 2MTAC & 1AKOA & 1MPGA \\
\hline 1XFFA & 2PIAA & $1 \mathrm{AH} 7 \mathrm{~A}$ & 1IIBA \\
\hline 1GPCA & 2RN2A & 13PKA & 1FDRA \\
\hline 1HLBA & $2 \mathrm{SCPA}$ & 1AMUA & $1 \mathrm{NBCA}$ \\
\hline 1IDOA & 3CHYA & 1MPGA & 1CIPA \\
\hline 1KTEA & $5 P 21 \mathrm{~A}$ & $1 \mathrm{~A} 48 \mathrm{~A}$ & 2TPSA \\
\hline 1LBAA & & 1A6QA & 1JFRA \\
\hline $1 \mathrm{LCLA}$ & & 2RN2A & 1A8LA \\
\hline 1MAIA & & 2TPSA & $2 \mathrm{GDMA}$ \\
\hline 1POCA & & 1AYLA & 2PIAA \\
\hline 1POTA & & 1THTA & \\
\hline 1RCFA & & 1NO3A & \\
\hline 1RECA & & 1STFI & \\
\hline 1RSYA & & 2MBRA & \\
\hline
\end{tabular}


Table 3.11. Regression accuracy for generic category using common PDB IDs between the 618 data set and the 215, 268, and 1363 data sets for the two regression models. The respective training set and test set used for each of these calculations are also presented in the table.

\begin{tabular}{|c|c|c|c|}
\hline \multirow{2}{*}{ Models } & \multicolumn{3}{|c|}{ Generic } \\
\hline & $\begin{array}{l}\text { Training }= \\
\text { Generic } 268 \\
\text { Test }=\text { Generic } \\
215\end{array}$ & $\begin{array}{l}\text { Training = Generic } \\
1363 \\
\text { Test }=\text { Generic } 215\end{array}$ & $\begin{array}{l}\text { Training= Generic } \\
268 \text { and Generic } 1363 \\
\text { Test }=\text { Generic } 215\end{array}$ \\
\hline $\mathrm{RSA}=\mathrm{E} 20$ & 62.2 & 61.6 & 62.01 \\
\hline $\mathrm{RSA}=\mathrm{E} 6$ & 68.4 & 68.5 & 68.5 \\
\hline $\mathrm{RSA}=\mathrm{FSHP}$ & 70.0 & 70.1 & 70.0 \\
\hline $\mathrm{RSA}=\mathrm{FSHP}+\mathrm{AA}$ & 71.1 & 71.3 & 71.3 \\
\hline $\mathrm{RSA}=\mathrm{AA}$ & 71.4 & 70.2 & 71.4 \\
\hline $\mathrm{RSA}=\mathrm{E} 20+\mathrm{AA}$ & 73.5 & 73.3 & 73.6 \\
\hline $\mathrm{RSA}=\mathrm{E} 6+\mathrm{AA}$ & 74.6 & 73.7 & 74.4 \\
\hline $\begin{array}{l}\mathrm{RSA}=\mathrm{E} 20+\mathrm{E} 6+ \\
\mathrm{AA}\end{array}$ & 74.6 & 73.7 & 74.4 \\
\hline $\mathrm{RSA}=\mathrm{E} 20+\mathrm{E} 6$ & 68.8 & 68.7 & 68.9 \\
\hline $\begin{array}{l}\mathrm{RSA}=\mathrm{E} 20+\mathrm{FSR}+ \\
\mathrm{FSHP}+\mathrm{AA}\end{array}$ & 74.6 & 73.7 & 74.1 \\
\hline $\begin{array}{l}\mathrm{RSA}=\mathrm{E} 6+\mathrm{FSR}+ \\
\mathrm{FSHP}+\mathrm{AA}\end{array}$ & 74.7 & 73.8 & 74.5 \\
\hline $\begin{array}{l}\mathrm{RSA}=\mathrm{E} 20+\mathrm{E} 6+ \\
\mathrm{FSR}+\mathrm{FSHP}+\mathrm{AA}\end{array}$ & 74.8 & 73.9 & 74.5 \\
\hline
\end{tabular}


Table 3.12. Regression accuracy for the protein binding category using common PDB IDs between the 618 data set and the 215,268 , and 1363 data sets for the two regression models. The respective training set and test set used for each of these calculations are also presented in the table.

\begin{tabular}{|l|l|l|l|}
\hline \multicolumn{1}{|c|}{ Models } & \multicolumn{3}{c|}{ Protein Binding } \\
\hline & $\begin{array}{l}\text { Training = } \\
\text { Protein Binding } \\
268 \\
\text { Test = Protein } \\
\text { Binding 215 }\end{array}$ & $\begin{array}{l}\text { Training = Protein } \\
\text { Binding 1363 } \\
\text { Test = Protein } \\
\text { Binding 215 }\end{array}$ & $\begin{array}{l}\text { Training= Protein } \\
\text { Binding 268 and } \\
\text { Protein Binding 1363 } \\
\text { Test = Protein Binding } \\
215\end{array}$ \\
\hline RSA=E20 & 60.9 & 60.9 & 60.9 \\
\hline RSA = E6 & 60.9 & 60.9 & 60.9 \\
\hline RSA = FSHP & 75.1 & 75.0 & 75.0 \\
\hline RSA = FSHP + AA & 74.5 & 76.2 & 74.5 \\
\hline RSA = AA & 73.4 & 73.7 & 73.4 \\
\hline RSA = E20 + AA & 74.8 & 76.0 & 75.6 \\
\hline RSA = E6 + AA & 75.2 & 77.3 & 76.7 \\
\hline $\begin{array}{l}\text { RSA =E20 + E6 + } \\
\text { AA }\end{array}$ & 75.0 & 77.2 & 76.3 \\
\hline RSA = E20 + E6 & 60.9 & 62.3 & 60.9 \\
\hline $\begin{array}{l}\text { RSA = E20 + FSR + } \\
\text { FSHP + AA }\end{array}$ & 76.0 & 76.2 & 77.0 \\
\hline $\begin{array}{l}\text { RSA = E6+ FSR + } \\
\text { FSHP + AA }\end{array}$ & 75.9 & 76.7 & 77.9 \\
\hline $\begin{array}{l}\text { RSA = E20 + } \\
\text { E6 +FSR +FSHP + } \\
\text { AA }\end{array}$ & 76.2 & 76.9 & 77.3 \\
\hline
\end{tabular}

Table 3.13 is the regression accuracy for oligomer category. Similar to the previous calculations, the oligomer category is the least well-predicted group because accuracies are consistently lower for each of the related models. The highest accuracy for the oligomer category is $73.6 \%$ for the RSA $=\mathrm{E} 20+\mathrm{E} 6+\mathrm{FSR}+\mathrm{FSHP}+\mathrm{AA}$ model using oligomers from 268 as the training and oligomers from 215 as the test set. 
Table 3.13. Regression accuracy for the oligomer category using common PDB IDs between the 618 data sets and the 215, 268, and 1363 data sets for the two regression models.

\begin{tabular}{|c|c|c|c|}
\hline \multirow[t]{2}{*}{ Models } & \multicolumn{3}{|c|}{ Oligomer } \\
\hline & $\begin{array}{l}\text { Training }= \\
\text { Oligomer } 268 \\
\text { Test = Oligomer } \\
215\end{array}$ & $\begin{array}{l}\text { Training }= \\
\text { Oligomer } 1363 \\
\text { Test }=\text { Oligomer } \\
215\end{array}$ & $\begin{array}{l}\text { Training= Oligomer } \\
268 \text { and Oligomer } \\
1363 \\
\text { Test }=\text { Oligomer } 215\end{array}$ \\
\hline $\mathrm{RSA}=\mathrm{E} 20$ & 61.5 & 60.4 & 61.2 \\
\hline $\mathrm{RSA}=\mathrm{E} 6$ & 68.3 & 68.5 & 68.3 \\
\hline $\mathrm{RSA}=\mathrm{FSHP}$ & 67.0 & 67.1 & 67.1 \\
\hline $\mathrm{RSA}=\mathrm{FSHP}+\mathrm{AA}$ & 69.4 & 69.4 & 68.5 \\
\hline $\mathrm{RSA}=\mathrm{AA}$ & 72.4 & 69.4 & 68.4 \\
\hline $\mathrm{RSA}=\mathrm{E} 20+\mathrm{AA}$ & 73.7 & 72.1 & 72.6 \\
\hline $\mathrm{RSA}=\mathrm{E} 6+\mathrm{AA}$ & 73.6 & 73.1 & 73.5 \\
\hline $\begin{array}{l}\mathrm{RSA}=\mathrm{E} 20+\mathrm{E} 6+ \\
\mathrm{AA}\end{array}$ & 73.6 & 73.0 & 73.4 \\
\hline $\mathrm{RSA}=\mathrm{E} 20+\mathrm{E} 6$ & 68.9 & 68.8 & 68.8 \\
\hline $\begin{array}{l}\mathrm{RSA}=\mathrm{E} 20+\mathrm{FSR}+ \\
\mathrm{FSHP}+\mathrm{AA}\end{array}$ & 73.1 & 72.1 & 72.9 \\
\hline $\begin{array}{l}\mathrm{RSA}=\mathrm{E} 6+\mathrm{FSR}+ \\
\mathrm{FSHP}+\mathrm{AA}\end{array}$ & 73.6 & 73.1 & 73.5 \\
\hline $\begin{array}{l}\mathrm{RSA}=\mathrm{E} 20+ \\
\mathrm{E} 6+\mathrm{FSR}+\mathrm{FSHP}+ \\
\mathrm{AA}\end{array}$ & 73.6 & 73.0 & 73.4 \\
\hline
\end{tabular}

Table 3.14 represents regression accuracies for the generic category excluding PDB IDs categorized as protein binding and oligomers. The key difference between Table 3.12 generic accuracy and Table 3.14 is that Table 3.12 includes protein binders and oligomers as part of the list. In contrast, for the calculations presented in Table 3.14, these two groups are removed. The highest accuracy for the generic category without the 
protein binders and oligomers categories is $76.0 \%$ for the RSA $=\mathrm{E} 20+\mathrm{E} 6+\mathrm{FSR}+$

FSHP + AA model.

Table 3.14. Regression accuracy for generic category excluding protein binding and oligomer categories using common PDB IDs between the 618 data sets with 215, 268 and 1363 data sets for the two regression models. The respective training set and test set used for each of these calculations are also presented in the table.

\begin{tabular}{|c|c|c|c|}
\hline \multirow[t]{2}{*}{ Models } & \multicolumn{3}{|c|}{ Generic - Protein Binding - Oligomer } \\
\hline & $\begin{array}{l}\text { Training = } \\
\text { Generic - } \\
\text { Protein } \\
\text { Binding- } \\
\text { Oligomer } \\
(268) \\
\text { Test }= \\
\text { Generic - } \\
\text { Protein } \\
\text { Binding - } \\
\text { Oligomer } \\
(215)\end{array}$ & $\begin{array}{l}\text { Training = } \\
\text { Generic - } \\
\text { Protein Binding } \\
\text { - Oligomer } \\
\text { (1363) } \\
\text { Test = Generic } \\
\text { - Protein } \\
\text { Binding - } \\
\text { Oligomer } \\
\text { (1363) }\end{array}$ & $\begin{array}{l}\text { Training= Generic } \\
\text { - Protein Binding } \\
\text { - Oligomer (268 } \\
\text { and 1363) } \\
\text { Test = Generic - } \\
\text { Protein Binding - } \\
\text { Oligomer (215) }\end{array}$ \\
\hline $\mathrm{RSA}=\mathrm{E} 20$ & 64.0 & 63.5 & 64.1 \\
\hline $\mathrm{RSA}=\mathrm{E} 6$ & 69.9 & 69.9 & 69.8 \\
\hline RSA $=$ FSHP & 70.7 & 70.9 & 70.8 \\
\hline $\begin{array}{l}\text { RSA = FSHP + } \\
\text { AA }\end{array}$ & 71.8 & 72.1 & 72.1 \\
\hline $\mathrm{RSA}=\mathrm{AA}$ & 71.9 & 70.7 & 70.7 \\
\hline $\begin{array}{l}\mathrm{RSA}=\mathrm{E} 20+ \\
\mathrm{AA}\end{array}$ & 74.6 & 74.3 & 74.4 \\
\hline $\mathrm{RSA}=\mathrm{E} 6+\mathrm{AA}$ & 75.4 & 74.7 & 75.4 \\
\hline $\begin{array}{l}\mathrm{RSA}=\mathrm{E} 20+\mathrm{E} 6 \\
+\mathrm{AA}\end{array}$ & 75.4 & 74.7 & 75.4 \\
\hline $\mathrm{RSA}=\mathrm{E} 20+\mathrm{E} 6$ & 70.1 & 70.1 & 70.0 \\
\hline $\begin{array}{l}\mathrm{RSA}=\mathrm{E} 20+ \\
\mathrm{FSR}+\mathrm{FSHP}+ \\
\mathrm{AA}\end{array}$ & 75.6 & 74.9 & 75.7 \\
\hline $\begin{array}{l}\mathrm{RSA}=\mathrm{E} 6+\mathrm{FSR} \\
+\mathrm{FSHP}+\mathrm{AA}\end{array}$ & 75.9 & 75.4 & 75.9 \\
\hline $\begin{array}{l}\text { RSA }=\text { E20 + } \\
\text { E6 +FSR } \\
+ \text { FSHP + AA }\end{array}$ & 76.0 & 75.4 & 75.9 \\
\hline
\end{tabular}




\section{Discussion}

The aggregate and correlation plots of the first of all of the data sets used for this work, the 268 training set, were presented in previous work by the Lustig group (Mishra, 2010). The earlier calculations derived by manually downloaded BLAST files for each PDB ID in the 268 data set has been presented for comparison and validation purposes of the automatically downloaded BLAST output files. The earlier manually presented data and the automated results presented in the results section of this thesis were identical, confirming the validity of the automated system. The automation of downloading BLAST files was then applied toward development of a larger data set. The 1363 training set filled the need for a larger training set. The 1363 set presented trends for all the distribution and correlation plots similar to both the automated and manual 268 training set. Also similar to the 268 training data set, the 1363 aggregate plots displayed the characteristics of two major regions for sequence homology parameters when plotted against inverse $\mathrm{C} \alpha$ packing density.

\subsection{Prediction of RSA}

The primary focus of this study was to accurately predict solvent accessibility of a given protein residue using a sequence qualitative predictor. The significance of linear regression in conjunction with the amino acid as qualitative predictors lies in the fact that, with a very limited number of sequence homology parameters, one can reasonably predict the likelihood of a residue being either buried or on the surface as a part of binary classification. The prediction accuracy ranged from $73-78 \%$ with models, and the combination of E20, E6, FSR, FSHP, and AA resulted in the highest accuracy achieved 
for most of the subset of calculations performed. The direct introduction of secondary subclass information as qualitative predictors did not improve prediction results. The sequence qualitative predictors directly introduce the actual query information into the analysis. By themselves, they offer significant prediction accuracy. However, our first attempts at nearest neighbor analysis using query sequence information, at least implicitly in averaging flanking of RSA values, did not improve accuracy (Nepal, R. and Lustig, B. San Jose State University, San Jose, CA. Unpublished work, 2011). This approach is a common one in k-nearest neighbor analysis (Joo et al., 2012; Sim et al., 2006).

There appears to be a fundamental limitation of prediction accuracy for solventaccessible residues. This involves having to deal with the association of solvent accessibility with quaternary structure. Similar intrinsic limitation is also noticed in secondary structure prediction (Kihara, 2005; Rost, 2001) with respect to tertiary structure. Though we calculated a $40 \%$ alignment score as a threshold, this is comparable to a sequence identity score of $40 \%$ (Yeh, 2005). But truncating sequences has its own problems including losing valuable information about the nature of certain substituted residues. Although sequence alignment helps identify differences between protein pairs of similar and non-similar structures in high sequence identity ( $>40 \%$ for long alignment), the signal gets less clear in $20-35 \%$ sequence identity (Jaroszewski et al., 2002; Rost, 1999; Schwarz et al., 2010). This blurring should have an impact on solvent accessibility.

There have been some additional improvements in solvent accessibility predictions using support vector machines (Adamczak et al., 2004) and other learning- 
based approaches, such as the random forest method to determine specific accessible surface area (Pugalenthi et al., 2012). These calculations still remain very computationally intensive and somewhat obscure in the physical interpretation of individual parameters. However, even under optimal threshold RSA criteria, the overall binary prediction limits remain just at or below the $78 \%$ accuracy. One parameter that could have an impact is the quality of a learning set. Assuming this source of error is addressed, the intrinsic limitations from coupling of local secondary and higher orders of 3D structure will likely still remain.

The most significant limitation for the prediction of residue solvent accessibility may be attributed to the coupling between residue surface accessibility and protein-protein contacts including quaternary structure. Although the majority of hydrophobic residues are found buried in the core of the protein structure, there are some hydrophobic residues on the surface of the protein involved in interaction with other proteins (Yan et al., 2008). Earlier work by the Lustig group presented a linear correlation between query hydrophobicity and inverse packing density in most of the Major Region I (Liao et al., 2005). On the other hand, $10 \%$ of Major Region II query residues were identified as strongly hydrophobic.

Although the accuracies calculated by linear regression model as described in this thesis are slightly lower, it is comparable to the most utilized RSA accuracy calculation reported in the literature (Adamczak et al., 2004). The former uses a complicated system built upon neural networks that involve multilayered feed. A continuous approximation of the real-value RSA using nonlinear regression was used with several feed-forward and 
recurrent neural networks that were then combined into a consensus predictor. We employed a simple two-step linear regression method for RSA prediction. Also, it was reported that the use of Position-Specific Iterative BLAST (PSI-BLAST) resulted in better accuracy. Our calculations resulted in similar accuracies with PSI-BLAST and did not improve the accuracy predictions.

We have found that a very limited number of parameters can result in significant prediction accuracy. These parameters include direct descriptor of actual sequence and the various Shannon entropies associated with their substitution. The inclusion of 20point sequence entropy displayed the flexibility of a given amino acid to change or mutate (Koehl and Levitt, 2001). Also noteworthy is that the addition of parameters involving the classification of strongly hydrophobic or small residues add some incremental value to the prediction. However, once one achieves accuracy approaching mid $70 \%$, additional parameter components add very little incrementally to the prediction accuracy.

Various surface accessibility prediction methods applying the two-state (buried and on the surface) have been recently developed. The use of SVM has been explored to improve solvent accessibility prediction accuracies (Kim and Park, 2003; Wang et al., 2007; Yuan et al., 2002). Typically, SVM constructs extended representation of data and then classifies them into groups. This requires the use of a training set to inform the boundaries of the classifier. Another method extensively applied to solvent accessibility prediction is the utilization of neural networks (Adamczak et al., 2004; Ahmad and Gromiha, 2002; Kim and Park, 2003; Rost and Sander, 1994). In neural networks 
methods, RSA is predicted using a non-linear regression method instead of a classification method. Unlike in SVM methods, here a continuous approximation and evaluation of the real-value RSA is produced instead, imposing an arbitrary threshold to the RSA (Adamczak et al., 2004). One such application in surface accessibility prediction is the fuzzy k-nearest method applied to sequence information (FKNN) by Sim et al. (2006); Joo et al. (2012) presented an additional application of the nearest neighbor method where a database is constructed based on sequence information of residues and its neighbors. Accuracy in all of these methods typically stays near $80 \%$, and this is consistent with the intrinsic problem at hand.

From a limited set of known quaternary contacts (Do, S.and Lustig, B. San Jose State University, San Jose, CA. Unpublished work, 2010) it was concluded that RSA prediction of residues on the surface is problematic. The RSA prediction of residues is challenging primarily due to the presence of hydrophobic patches on the surface of the protein. However, it is known in general that $50 \%$ of the globular protein's surface is non-polar, making "hydrophobic patches" inevitable even if protein does not interact with other proteins (Eisenhaber and Argos, 1996; Lins et al., 2003). Although the majority of hydrophobic residues are found buried deep within a protein structure, some are present on the surface of the protein interacting with other complexes. To address this issue in solvent accessibility prediction, Bahadur et al. (2004) described a method in which surface residues are divided into two groups: the "core" and the "rim" set. This core is not necessarily what is descriptive of the core in buried residues of the folded protein. The core residues present in surface-accessible patches include interfaces with quaternary 
interaction, and a mere presence of one buried interface atom is enough to categorize a given residue as a core residue (Chakrabarti and Janin, 2002).

The incorporation of 618 categorical PDB IDs (Petit et al., 2007) was performed in an attempt to improve RSA prediction accuracies. These proteins are in context of an algorithm to predict, from X-ray structure, functionally relevant patches on the surface of various classes of proteins. It was observed that, out of all the calculations performed, our method consistently resulted in a better prediction for the protein binding category better than any other group, whereas the oligomer group dependently resulted in lower accuracies. The difference between the highest protein binding category and the highest oligomer category was about $4.5 \%$. Noteworthy, the remaining of the 618 PDB IDs excluding protein binding PDB IDs, and oligomeric IDs, resulted in prediction accuracies better than the oligomeric category, but not as good as protein binding. Here the difference between protein binding and this group was around $2 \%$.

In this thesis we have outlined a reliable RSA prediction method, and further enhancements in this scheme have a potential to be a novel simple binary RSA predictions method as a gold standard for the field. Incorporation of a larger training data set (i.e., 1363) did not yield a drastic improvement over RSA prediction. Unlike Joo et al. (2012), who claimed the larger data set improved results drastically, our results do not indicate such. The original size of the data set for the 1363 PDB IDs in the 1363 training set was 6511 proteins (Bondugula et al., 2011), and similar PISCES culling parameters were applied to this list as stated in the Joo et al. paper. After the application of PISCES, the original list of 5157 (subset of the 6511 PDBs that were part of NCBI PDB library) 
was reduced to 1363 of structurally diverse set. Even though, Joo et al. (2012) found 5717 proteins, after application of the culling service PISCES, it is hard to imagine that the list is not structurally redundant.

Our results have indicated that dividing the proteins into various structural categories holds itself as a promising new direction for RSA predictions. The role of solvent accessibility in protein binding categories and oligomers needs to be further investigated to discover why the latter results in poor prediction accuracy while the former does not. Currently for this work, the incorporation of structural information (i.e., protein binding, oligomers) to the existing data sets $(268,1363$, and 215$)$ could only be applied to PDB IDs that were present in the 618 data set. As a future direction to the project, a reliable method to apply structure information to every PDB ID in the existing data sets needs to be assimilated. Once this has been done, the RSA prediction calculations need to be recalculated, and any changes observed could be noteworthy. Also, multiple stages have displayed some level of advantage in terms of RSA prediction with SVM and linear regressions (Adamczak et al., 2005). A second-stage implementation of the existing first-stage qualitative predictor methodology may present itself as a new improvement to the existing systems. 


\section{Future Studies}

Additional studies to further the work described in this thesis are listed below:

- Investigate the reasons why the protein binding category consistently results in higher prediction accuracy, whereas the oligomer category yields a lower range of prediction accuracy.

- Protein binding and oligomeric classifications should be made for all of the three different data sets $(268,1363$, and 215$)$.

- A second stage regression method should be incorporated into the existing regression approach. Our preliminary results indicated the incorporation of a distant homolog as a second filter yielded higher accuracy; this approach needs to be further analyzed.

- The possibility of application of logistic regression where an outcome is predicted on the basis of categorical dependent variable in the presence of one or more predictor variables should be explored for better accuracy predictions.

- Further in-depth exploration of the current method should be implemented to recognize amino acid residues and RSA values that are most mispredicted with the existing method.

- Further investigate a larger training NACCESS threshold than the 20 that is currently being used. 


\section{Conclusions}

Query-based qualitative predictors with protein sequence information were utilized to predict protein residue solvent accessibility. An automated system to download, integrate, and analyze various homology-based parameters and calculations was developed. The manual and automated characterization were deemed to be identical, thereby validating the later method. A novel and larger training data set (1363 training) was developed. The distribution plots of both the 268 and 1363 training data sets displayed a bimodal frequency distribution of residues, indicating the presence of highly hydrophobic residues on the surface. This is consistent with the notion of intrinsic limitations in predicting surface-accessible residues with only one chain.

A total of 12 main regression models utilizing various combinations of the two types of entropies (E20 and E6), homology-based parameters (FSR, FSHP) as quantitative predictors, and direct 20 amino acid information as qualitative predictors were created. Our results indicate consistently that E20 + E6 + FSR + FSHP + AA resulted in the highest accuracy compared to all the other models for all the tests performed. Interestingly, 6-point entropy (E6) with the qualitative predictor (AA) resulted in better prediction than the use of 20-point entropy (E20). In fact, in some sets of linear regression accuracy calculations, E6 + AA did almost as well as the best model. And breaking the FSR qualitative predictor into its two individual components (FA and FG) did not improve prediction accuracy. The incorporation of a larger training data set did not improve the accuracy prediction of the test set but actually resulted in accuracies comparable to the smaller training data set. 
Categorical regressions, where the proteins are divided into groups such as protein binding and oligomer, bring about some interesting information. The linear regression method imposed in this thesis seemed to consistently yield higher prediction accuracy for the protein binding category. On the other hand, the oligomeric category resulted in slightly lower prediction accuracies. 


\section{Bibliography}

Adamczak, R.; Porollo, A.; Meller, J. Proteins 2004, 56, 753-767.

Adamczak, R.; Porollo, A.; Meller, J. Proteins 2005, 59, 467-475.

Ahmad S.; Gromiha, M.M. Bioinformatics 2002, 18, 819-824

Bahadur, R. P.; Chakrabarti, P.; Rodier, F.; Janin J. J. Mol. Biol. 2004, 336, 943-955.

Berezovsky. I. N.; Trifonov, E. N. J. Mol. Biol. 2001, 307, 1419-1426.

Berman, H. M.; Westbrook, J.; Feng, Z.; Gilliland, G.; Bhat, T. N.; Weissig, H.;

Shindyalov, I. N.; Bourne, P. E. Nucl. Acids. Res. 2000, 28, 235-242.

Berman, H. Structure. 2008, 16, 16-18.

Bondugula, R.; Wallqvist, A.; Lee, M. S. Protein Eng. Des. Sel. 2011, 24, 455-461.

Carugo, O. Protein Eng. 2000, 13, 607-609.

Chakrabarti, P.; Janin, J. Proteins 2002, 47, 334-343.

Chayen, N. E.; Saridakis, E. Nature Methods. 2008, 5, 147-153.

Daggett, V.; Fersht, A. Nature Rev. Mol. Cell Biol. 2003, 4, 497-502.

Dale, G. E.; Oefner, C.; d'Arcy, A. J. Struct. Biol. 2003, 142, 88-97.

Eisenhaber, F.; Argos, A. Protein Eng. 1996, 9, 1121-1133.

Eswar, N.; Marti-Renom, M. A.; Webb, B.; Madhusudhan, M. S.; Eramian, D.; Shen, M.; Pieper, U.; Sali. Curr. Protoc. Bioinformatics 2006, Supplement 15, 5.6.1-5.6.30.

Gluehmann, M.; Zarivach, R.; Bashan, A.; Harms, J.; Schluenzen, F.; Bartels, H.; Agmon, I.; Rosenblum, G.; Pioletti, M.; Auerbach, T.; Avila, H.; Hansen, H. A. S.; Franceschi, F.; Yohnath, A. Methods 2001, 25, 292-302.

Graebsch, A.; Roche, S.; Kostrewa, D.; Soding, J.; Niessing, D. PLos One. 2010, 510.

Guharoy, M.; Chakrabarti, P. Natl. Acad. Sci. USA 2005, 102, 15447-15452.

Hellevik, O. Qual. Quant. 2009, 43, 59-74. 
Hsieh, M.; Collins, E. D.; Blomquist, T.; Lustig, B. J. Biomol. Struct. Dyn. 2002, 20, 243-251.

Hubbard, S. J.; Campbell, S. F.; Thornton, J. M. J. Mol. Biol.1991, 220, 507-530.

Hubbard, S. J.; Thornton, J. M. NACCESS, Computer Program, Department of

Biochemistry and Molecular Biology, University College

London.http://www.bioinf.manchester.ac.uk/naccess/, 1993.

Jaroszewski, L.; Li, W.; Godzik, A. Protein Sci. 2002, 11, 1702-1713.

Joo, K.; Lee, S. J.; Lee, J. Proteins 2012, 80, 1791-1797.

Kallblad, P.; Dean, P. M. Proteins 2004, 56, 693-703.

Kihara, D. Protein Sci. 2005, 14, 1955-1963.

Kim, H.; Park, H. 2003, Protein Eng. 2003, 16, 553-560.

Koehl, P.; Levitt, M. Proc. Natl. Acad. Sci. USA 2002, 99, 1280-1285.

Kutner, M. H, Nachshelm, C. J, Neter, J. Applied Linear Statistical Models; McGrawHill, New York, CA, 2004, Vol. 5, Chapter 8.

Liao, H.; Yeh, W.; Chiang, D.; Jernigan, R. L.; Lustig, B. Protein Eng. 2005, 18, 59-64.

Lins, L.; Thomas, A.; Brasseur, R. Protein Sci.2003, 12, 1406-1417.

Mishra, R. Characterization of Protein Residue Structural Accessibility Using Sequence Entropy; M.S. Thesis, San Jose State University, San Jose, CA, 2010.

Mizianty, J.; Kurgan, L. Bioinformatics 2011, 27, i24-i33.

Mizianty, M. J.; Kurgan, L. A. Protein Pept. Lett. 2012, 19, 40-49.

Naderi-Manesh, H.; Sadeghi, M.; Arab, S.; Movahedi, A. A. M. Proteins 2001, 42, $452-$ 459.

National Center for Biotechnology Information (NCBI), Protein Blast (BLASTP), http://www.ncbi.nlm.nih.gov. Accessed 2011.

Oliveira, L.; Paiva, A. C. M.; Vriend, G. ChemBioChem. 2002, 3, 1010-1017.

Panchenko, A. R.; Kondrashov, F.; Bryant, S. Prot. Sci. 2004, 13, 884-892.

Petrova, N. V.; Wu, C. H. BMC Informatics 2006, 7, 313-323. 
Pettit, F. K.; Tsa, A.; Bowie, J. U. J. Mol. Biol. 2007, 369, 863- 879.

Porollo, A.; Meller, J. Proteins 2007, 66, 630-645.

Poupon, A.; Mornon, J. P. FEBS Lett. 1999, 452, 283-289.

Pugalenthi, G.; Kandaswamy, K. K.; Chou, K.; Vivekanandan, S.; Kolatkar, P. Protein Pept. Lett. 2012, 19, 50-56.

Richardson, C. J.; Barlow, D. J. Prot. Engr. 1999, 12, 1051-1054.

Rose, D. A.; Nepal, R.; Mishra, R.; Lau, R.; Gholizadeh, S.; Lustig, B. Conference in Twenty-Secondary International Workshop on Database and Expert Systems Applications, DEXA, Toulouse, France, September 29, 2011; Morvan, F.; Tjoa, A. M.; Wagner, R. R., Eds.; IEEE Computer Society, Los Alamitos, 2011, 70-74.

Rost, B. J. Struct. Biol. 2001, 134, 204-218.

Rost, B.; Sander, C. Proteins 1994, 20, 216-226.

Savchenko, A.; Yeh, A.; Khachatryan, A.; Evdokimova, E.; Pavlova, M.; Semes, A.; Northey, J.; Beasley, S.; Lan, N.; Das, R.; Gerstein, M.; Arromith, C.H.; Edwards, A.M. Proteins 2003, 50, 392-399.

Schwarz, R.F.; Fletcher, Wi.; Forster, F.; Merget, B.; Wolf, M.; Schultz, J.; Markowetz, F. PLoS One. 2010, 5, 12.

Shenkin, P. S.; Erman, B.; Mastrandrea, L.D. 1991, Proteins, 11, 297-313.

Sim, J.; Kim, S.Y.; Lee, J. Bioinfomatics. 2006, 21, 2844-2849.

Structural Classification of Proteins (SCOP), http://scop.mrc-lmb.cam.ac.uk/scop/. Accessed 2011.

Valdar, W. S. J. Proteins 2002, 48, 227-241.

Wagner, M.; Adamczak, R.; Porollo, A.; Meller, J. J. Comput. Biol. 2005, 12, 355-369.

Wang, G.; Roland L. Dunbrack, J. Bioinformatics 2003, 19, 1589-1591.

Wang, J.Y.; Lee, H.; Ahmad, S. Proteins 2007, 68, 82-91.

Yan, C.; Terribilini, M.; Wu, F.; Jernigan, R. L.; Dobbs, D.; Honavar, V. BMC Bioinformatics 2006, 7, 262-271.

Yan, C.; Wu, F.; Jerrigan, R. L.; Dobbs, D.; Honavar, V. Prot J. 2008, 27, 59-70. 
Yeh, W. Detailed analysis of protein sequence entropy, hydrophobicity, and flexibility; MS Thesis, San Jose State University, San Jose, 2005.

Yuan, Z.; Burrage, K.; Mattick, J.S. Proteins 2002, 48, 566-570. 


\section{Appendices}

\section{A. Program Listings}

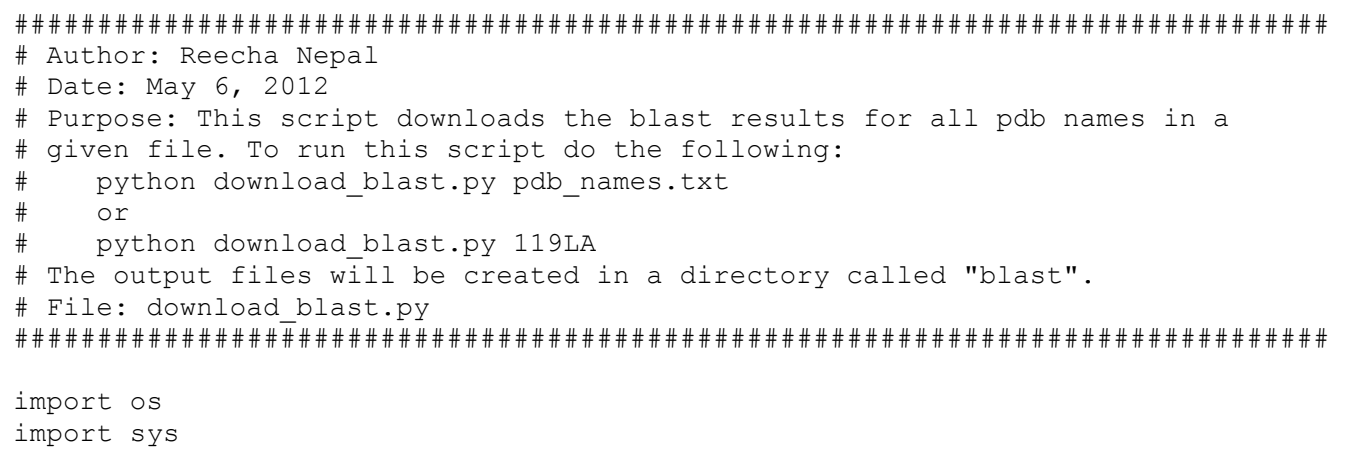

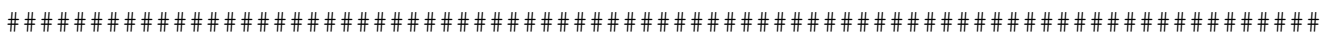
\# Returns the blast results for the given GI number.

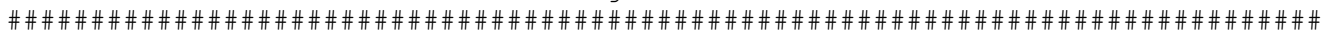
def download blast for gi number(gi number):

from Bio.Blast import NCBIWWW

blast result handle = NCBIWWW. qblast('blastp', 'nr', gi number, ।

format_type="Text", alignments="1000", descriptions="10000",

blast_text $=$ blast_result handle.read $($ ) hitlist size="10000")

blast result handle.close()

return̄ blast_text

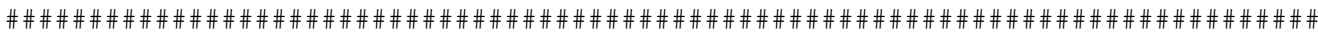
\# Reads the fasta file for the given pdb name.

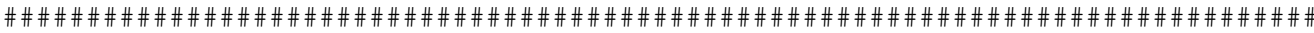
def read fasta file for pdb name (pdb name) :

file_name = os.path.join ("fasta", pdb_name + ".fasta")

if not os.path.exists (file name):

print "Error: Couldn't find file:", file name

print "Did you run the download_fasta.py script?"

sys.exit $(-1)$

input file handle = open (file name, "r")

input_data ${ }^{-}=$input_file_handlēeread ()

input file handle. $\bar{c}$ lose ()

returñ inpūt_data

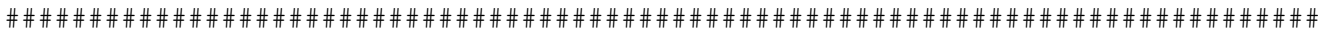

\# Gets the output path for the given pdb_name. For example, if the PDB name

\# is 1HGXA then the output path would be:

\# blast/1HGXA.txt

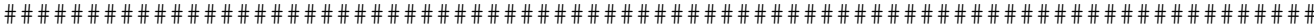

def get_output_path (pdb_name):

\# put the file in a directory named "blast"

if not os.path.exists ("blast") : os.makedirs ("blast")

output path = os.path.join("blast", pdb name + ".txt")

return output_path

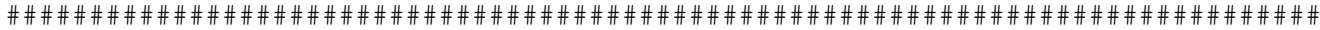
\# Saves the blast data with the fasta data at the top.

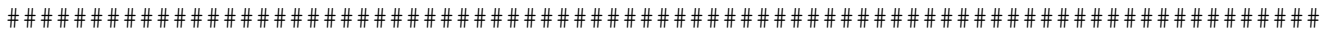




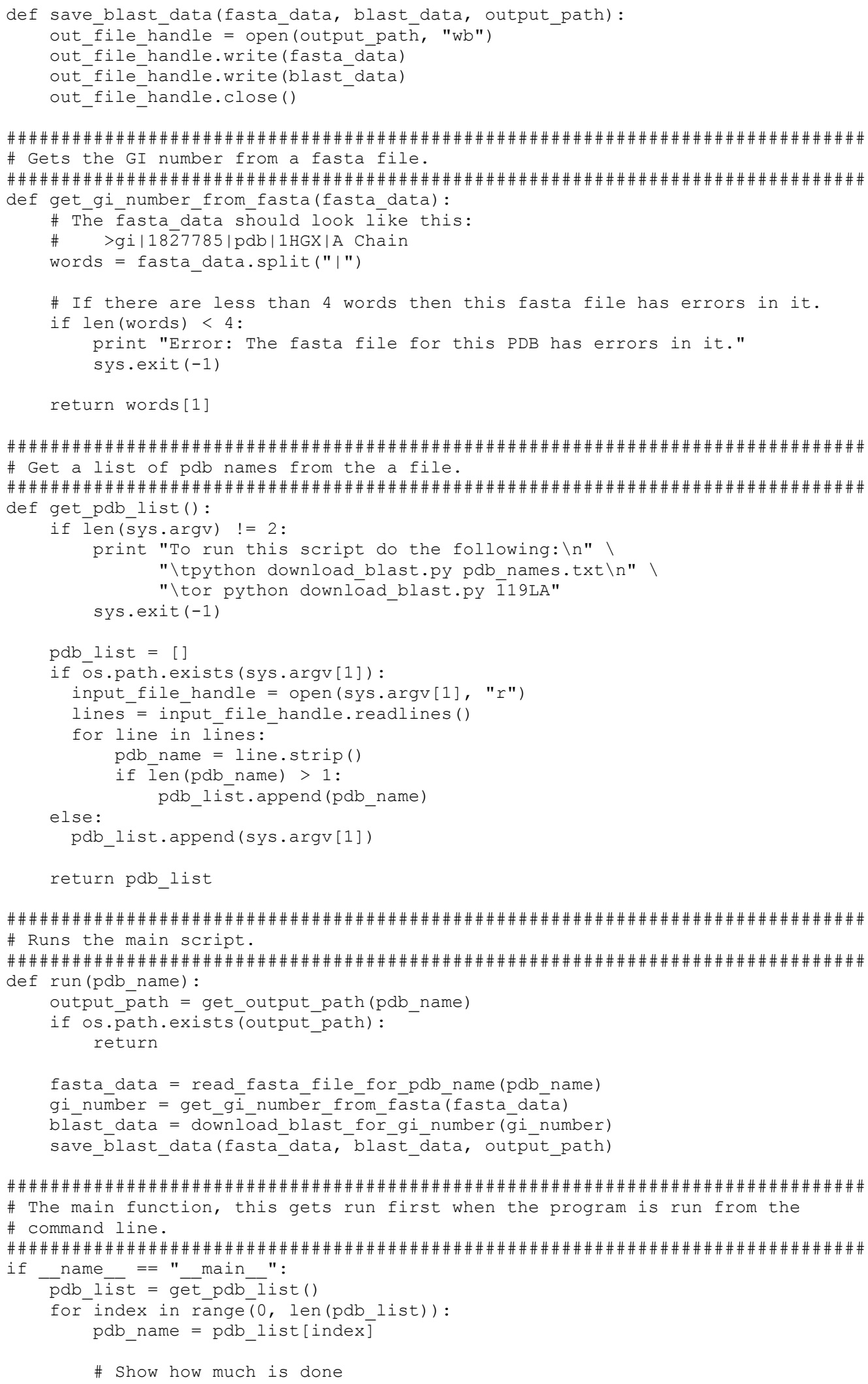




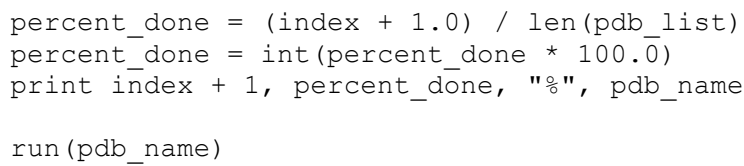




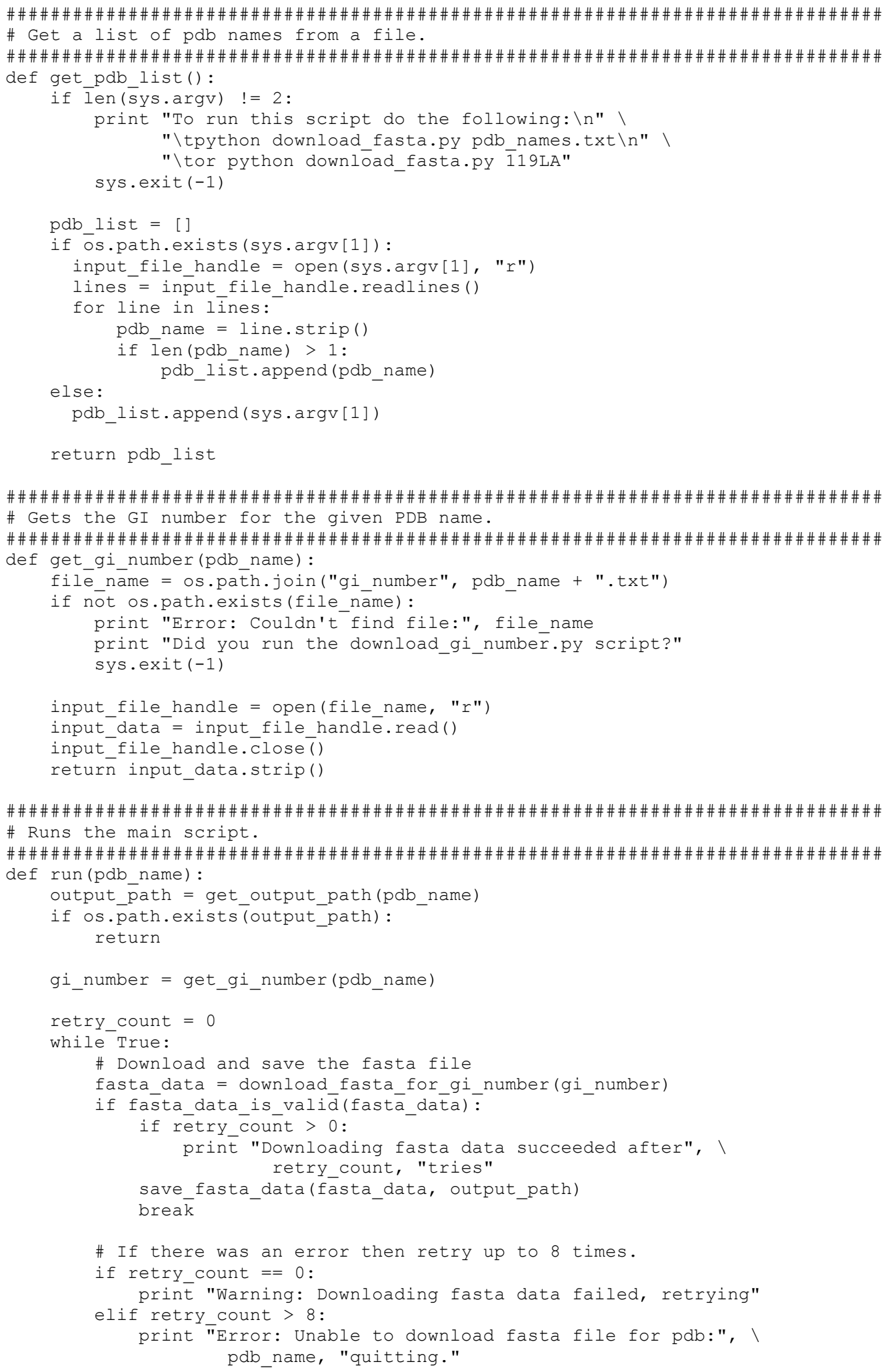

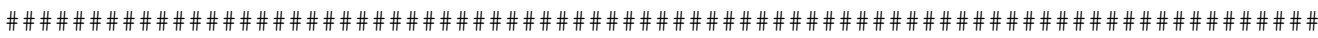




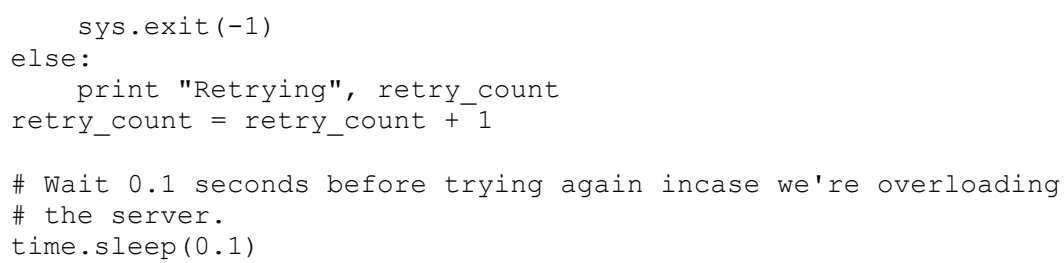

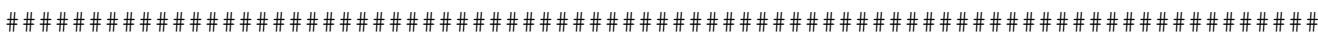
\# The main function, this gets run first when the program is run from the \# command line.

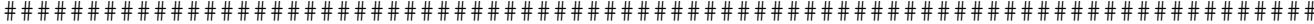

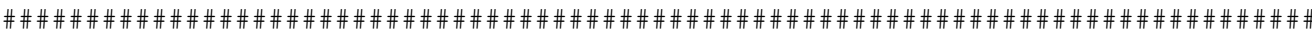
\# Gets the output path for the given pdb name. For example, if the PDB name 


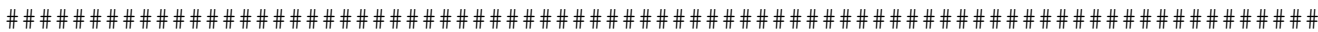
\# Save the GI number in a directory called gi_number.

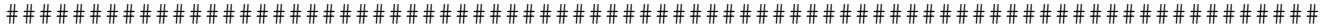

def save_gi_number_data(gi_number, output_path):

out $\overline{f i l e}$ handle $=$ open (output path, "wb")

out file_handle.write (gi_number)

out_file_handle.close()

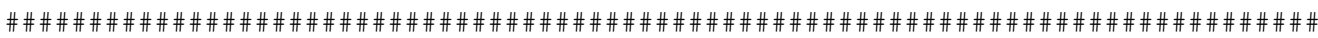

\# Checks if the GI number data is valid. Valid data should look like this:

$<$ esearchResult $>$

$<$ Count $>1</$ Count $>$

$<$ RetMax $>1</$ RetMax $>$

$<$ RetStart $>0</$ RetStart $>$

$<$ IdList $>$

$\langle$ Id $>157829547</$ Id $>$

$</$ IdList $>$

$<$ Translationset/>

$<$ QueryTranslation/>

$</$ esearchResult $>$

If there's a server error then we sometimes get data that looks like this:

Error:Cannot connect to database

\# If the data is valid then returns the GI number.

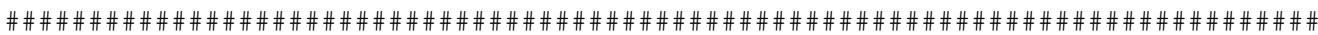

def parse gi number from data (data):

string $=$ data.decode ("utf-8")

if string.find ("eSearchResult") $=-1$ :

return -1

dom = parsestring (data)

id_list $=$ dom.getElementsByTagName ("Id")

if not id list or len(id list) $==0$ :

return -1

return id_list[0].childNodes[0].nodeValue

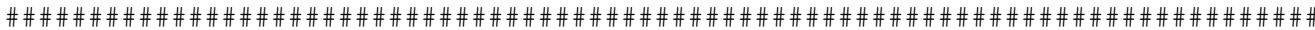
\# Get a list of pdb names from a file.

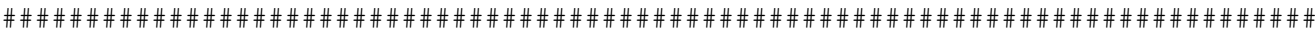
def get pdb list ():

if $\bar{l}$ en ( $\bar{s} y s \cdot a r g v) \quad !=2$ :

print "To run this script do the following: \n" \

"\tpython download_gi_number.py pdb names.txt \n" \

"\tor python download gi number.py $119 \mathrm{LA} "$

sys.exit $(-1)$

pdb_list $=[]$

if os.path.exists (sys.argv[1]):

input_file handle = open (sys.argv[1], "r")

lines ${ }^{-}=$input file handle.readlines()

for line in lines:

pdb name = line.strip ()

if len (pdb name) > 1:

else:

pdb_list.append (pdb_name)

pdb_list.append (sys.argv[1])

return pdb_list

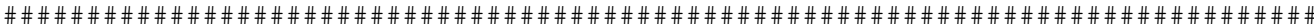

\# Runs the main script.

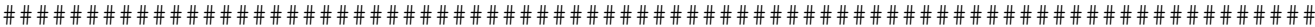

def run(pdb_name):

output path = get output path (pdb name)

if os.path.exists (output_path):

return 


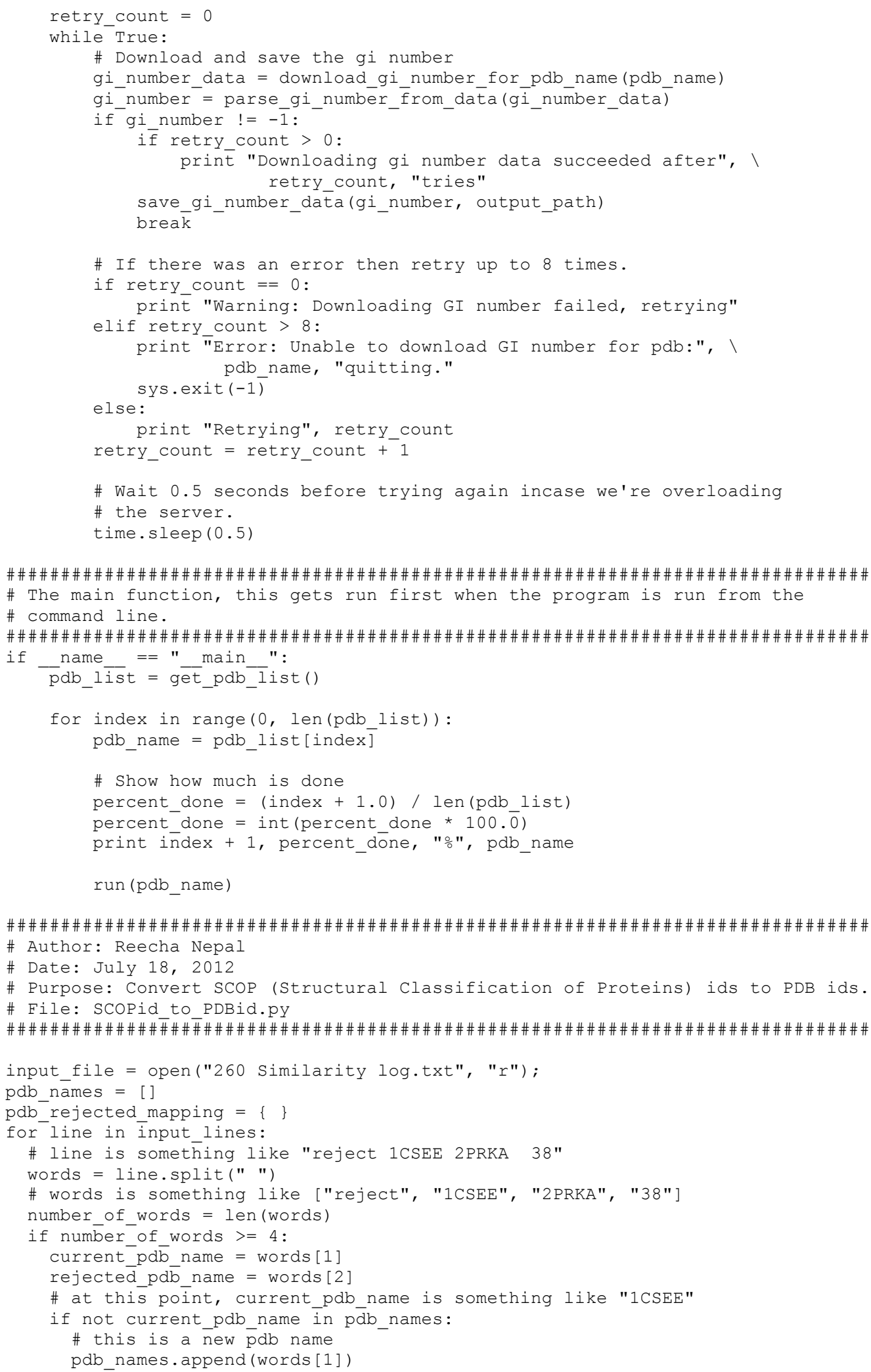




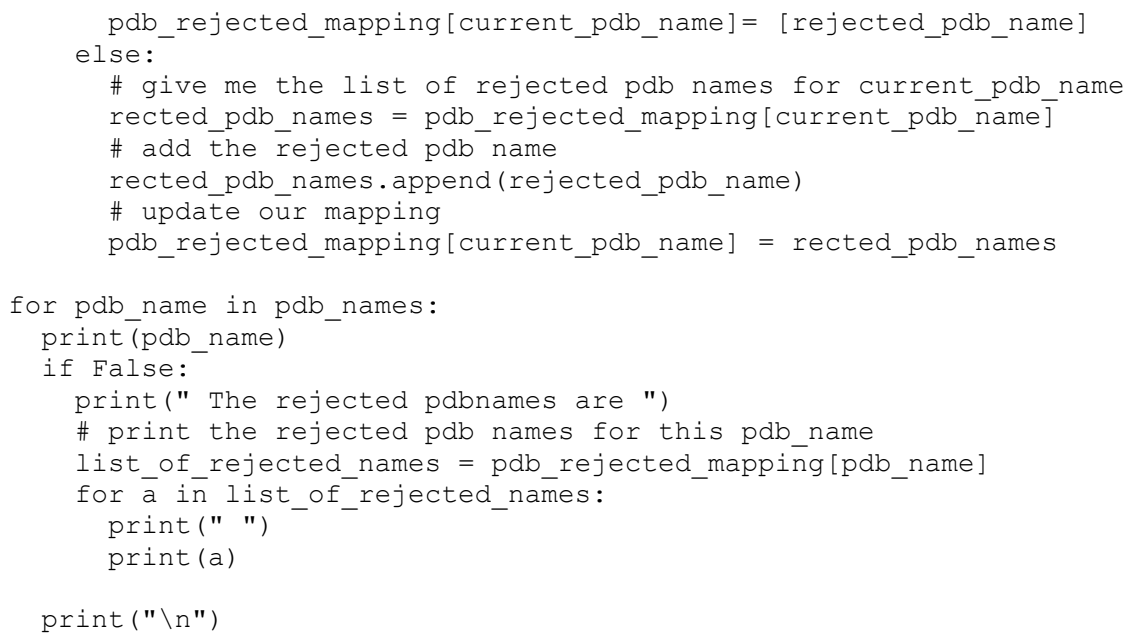




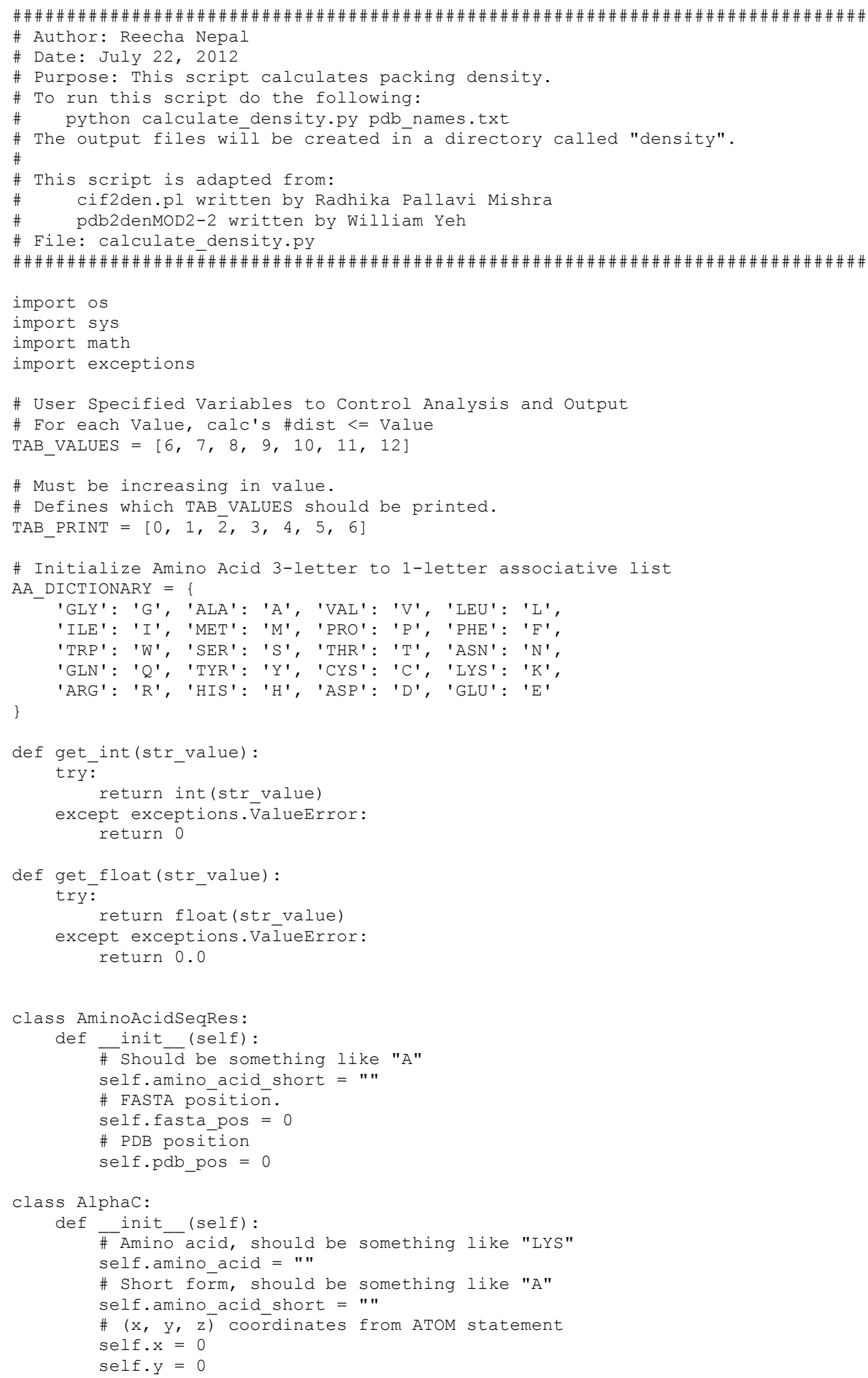




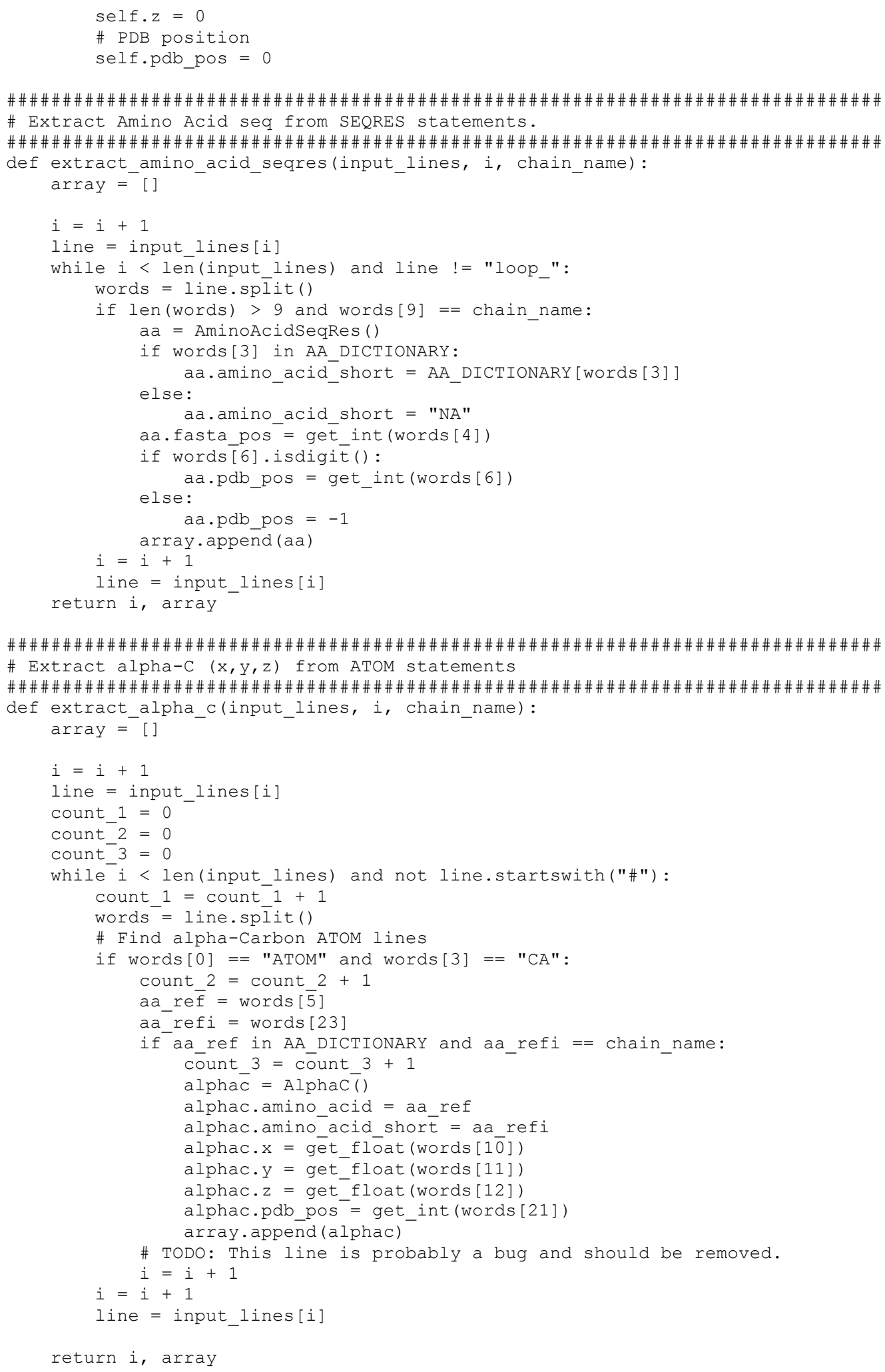

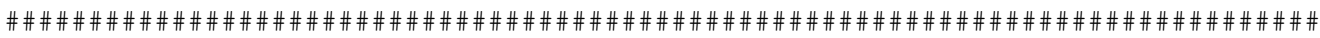




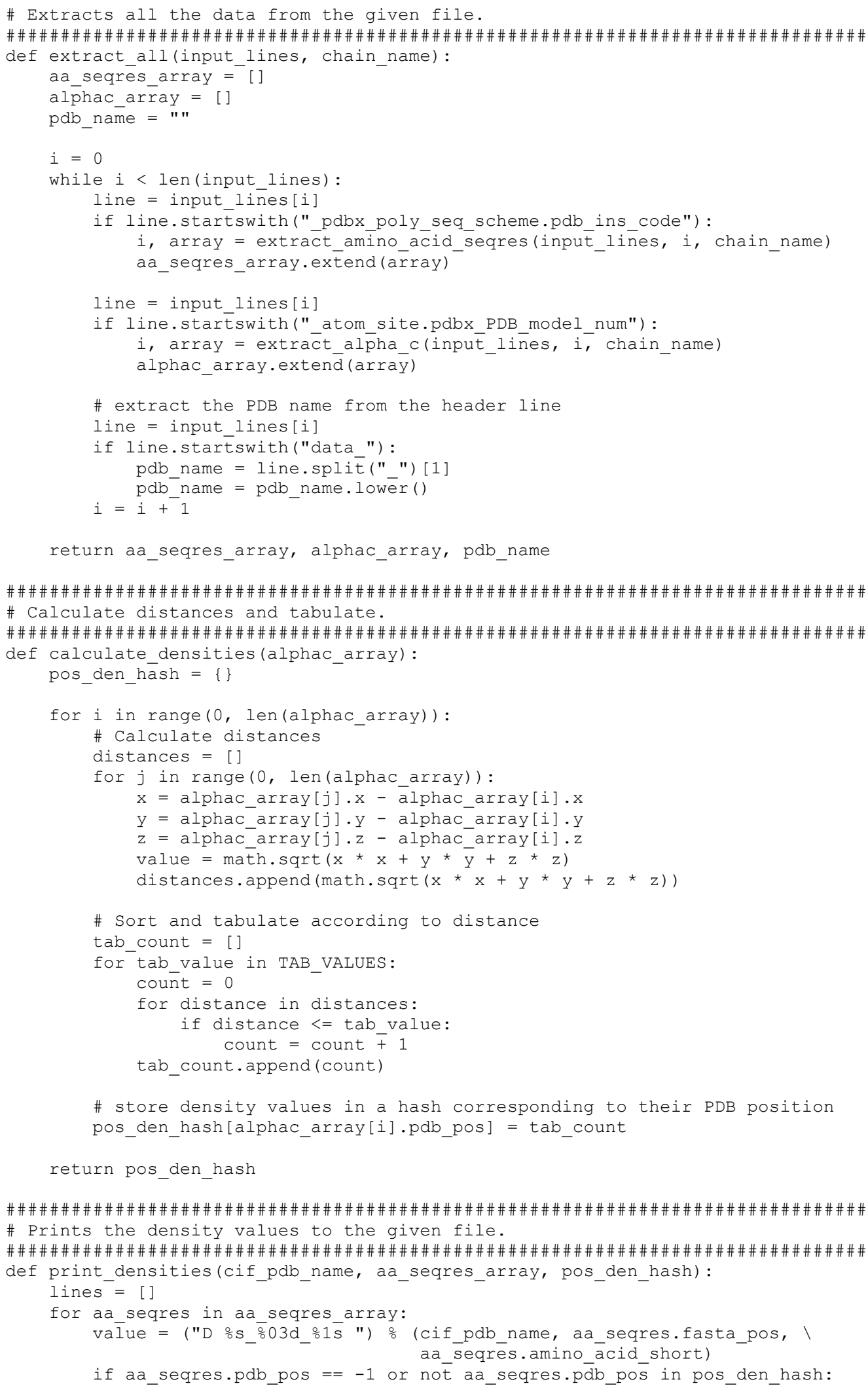

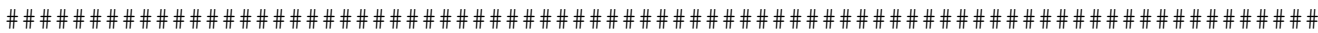
\# Prints the density values to the given file.

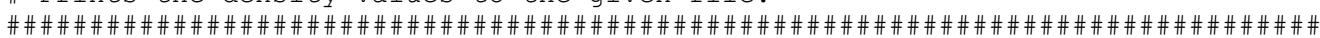
def print densities (cif pdb name, aa seqres array, pos den hash): 


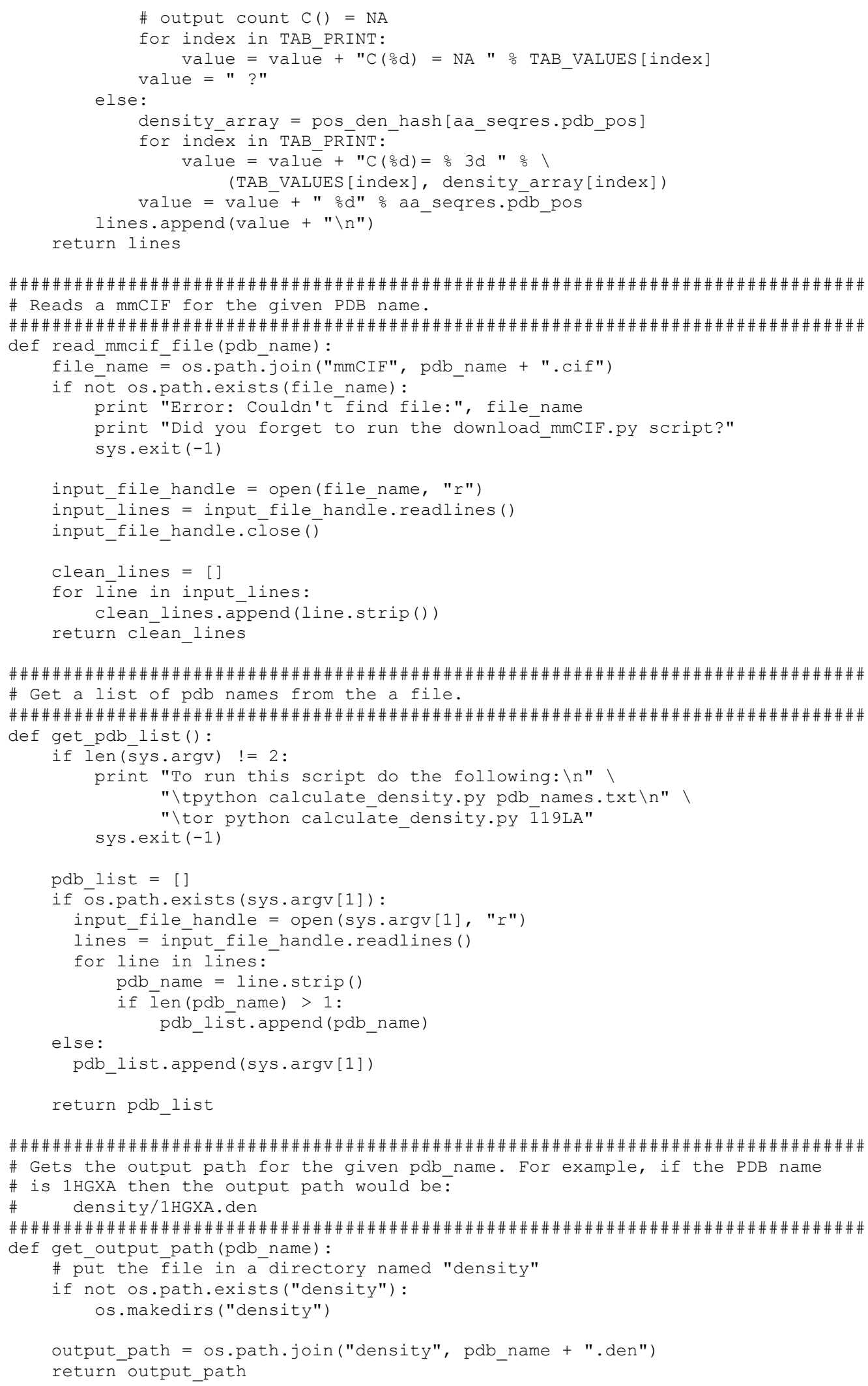




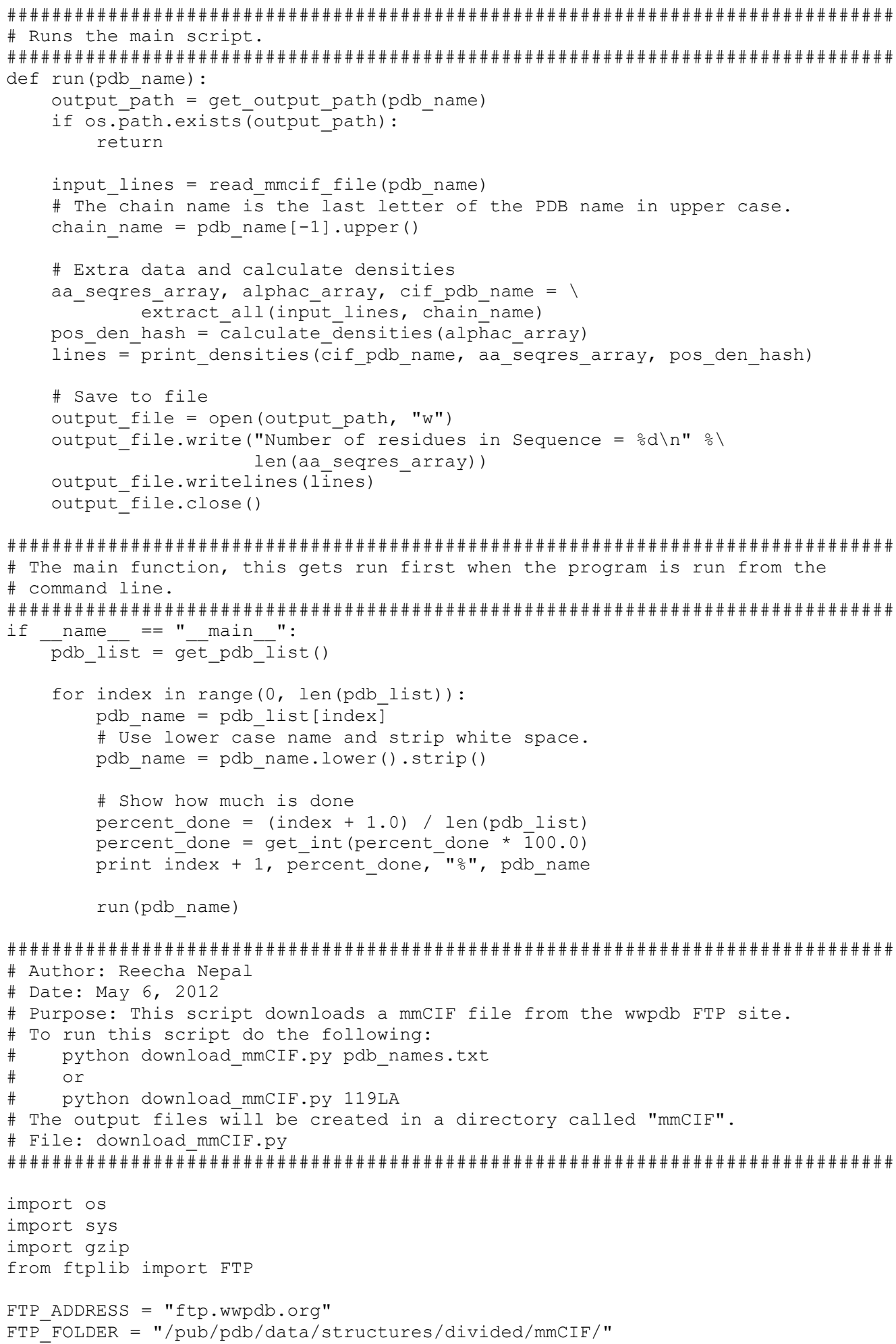

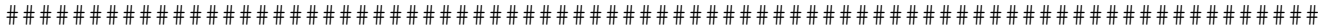

\# Author: Reecha Nepal

\# Date: May 6, 2012

\# Purpose: This script downloads a mmCIF file from the wwpdb FTP site.

\# To run this script do the following:

\# python download_mmCIF.py pdb_names.txt

\# or

\# $\quad$ python download mmCIF.py 119LA

\# The output files wīll be created in a directory called "mmCIF".

\# File: download mmCIF.py

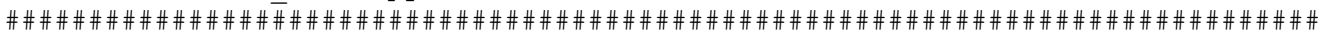

import os

import sys

import gzip

from ftplib import FTP

FTP_ADDRESS = "ftp.wwpdb.org"

FTP_FOLDER = "/pub/pdb/data/structures/divided/mmCIF/"

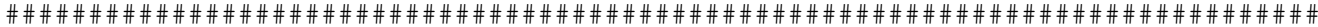

\# Gets the output path for the given pdb_name. For example, if the PDB name 


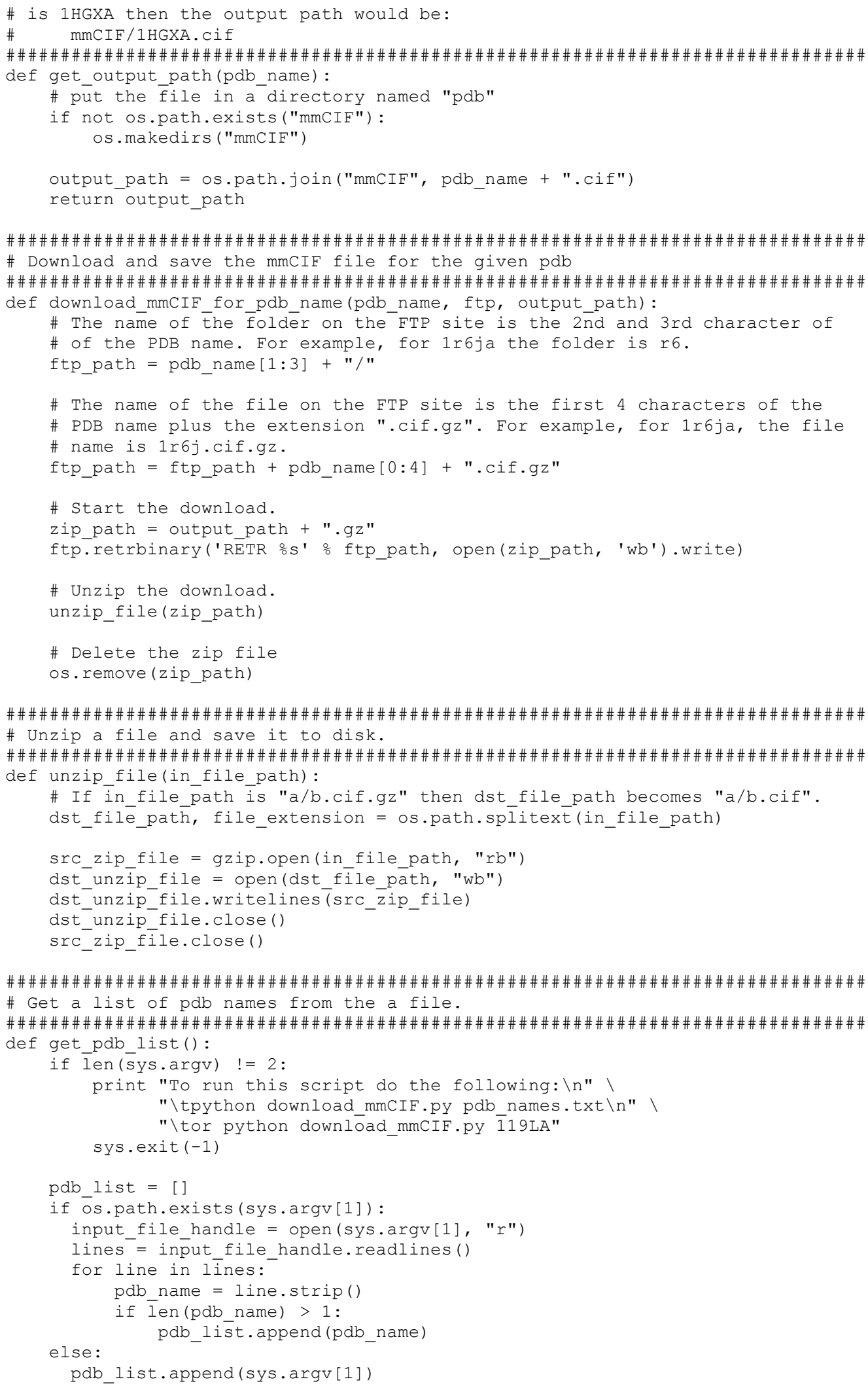




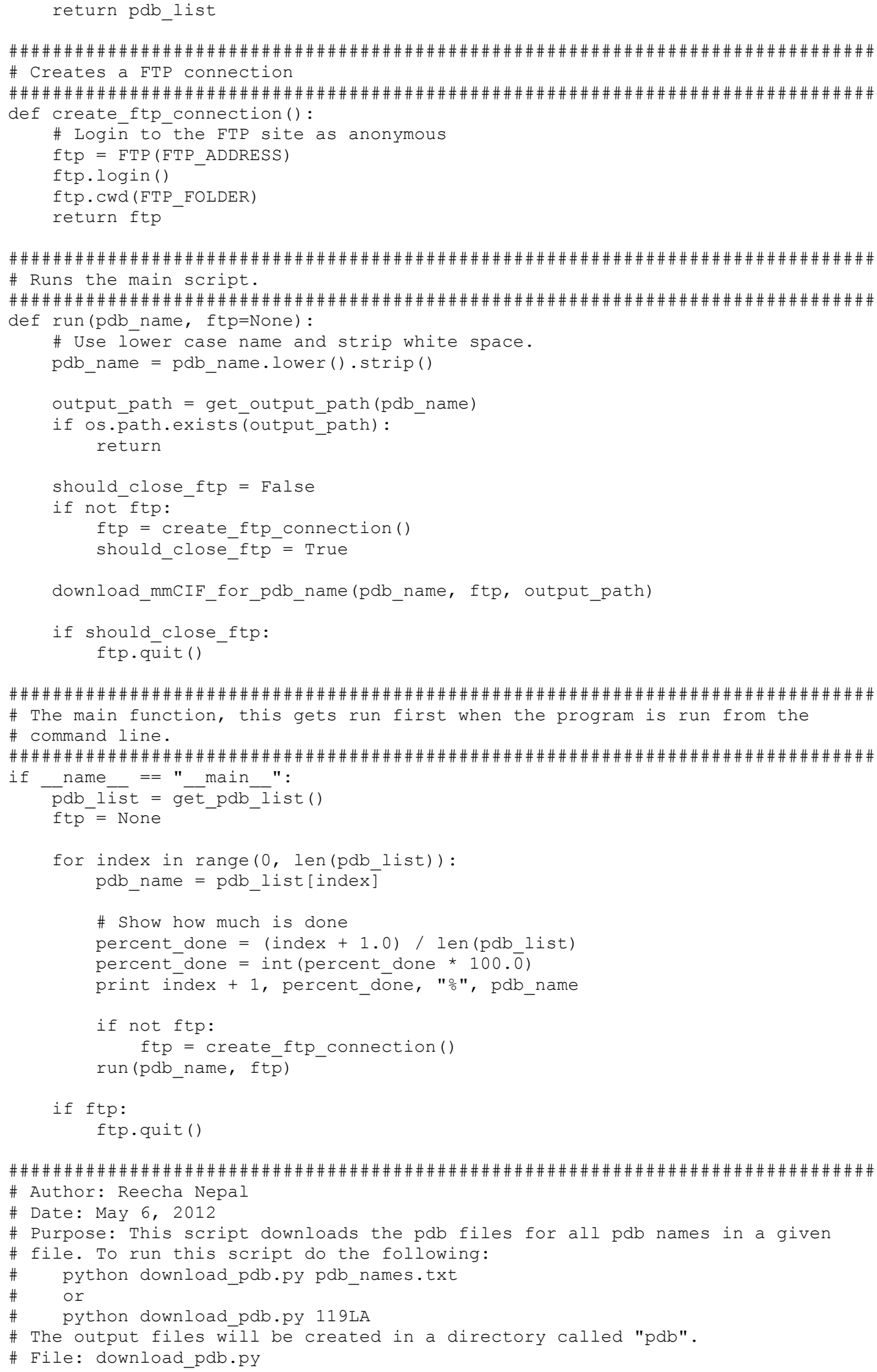


from urlib import urlopen

import os

import time

import sys

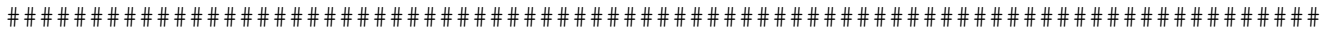
\# Download and return the pdb file for the given pdb name.

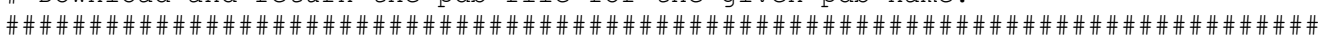
def download pdb for pdb name (pdb name):

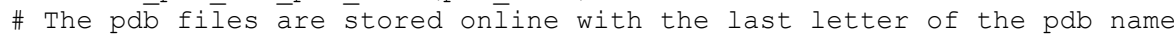

\# removed. For example, 119LA becomes 119L.pdb

pdb_name $=$ pdb_name $[:-1]$

url ${ }^{-}=$"http://Www.pdb.org/pdb/files/" + pdb name + ".pdb"

url file handle = urlopen (url)

pdb ${ }^{-}$data ${ }^{-}=$url file handle.read ()

url_file_handlēelose()

retürn pōb_data

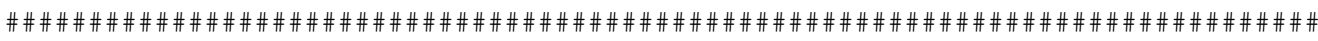
\# Gets the output path for the given pdb name. For example, if the PDB name

\# is 1HGXA then the output path would be:

\# $\quad \mathrm{pdb} / 1 \mathrm{HGXA}$. pdb

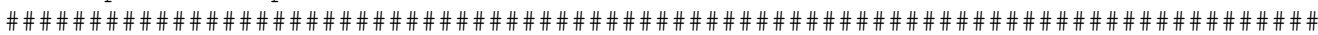
def get output path (pdb name) :

\# put the file in a directory named "pdb"

if not os.path.exists ("pdb"):

os.makedirs ("pdb")

output_path = os.path.join ("pdb", pdb_name + ".pdb")

return output_path

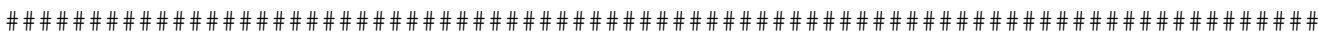

\# Save the pdb file in a directory called pdb.

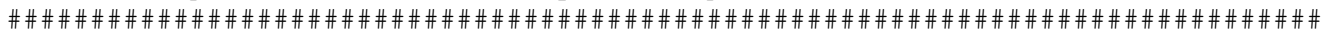

def save_pdb_data (pdb_data, output_path):

out_file_handle $=$ open (output_path, "wb")

out_file_handle.write (pdb_datā)

out_file_handle.close()

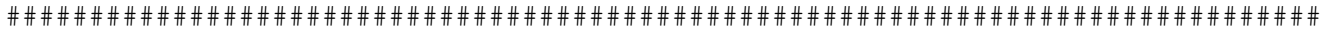

\# Checks if the pdb data is valid. A valid pdb data should look like this:

\# $\quad$ HEADER HYDROLASE (O-GLYCOSYL) 28-MAY-93 $119 \mathrm{~L}$

\# If there's a server error then we sometimes get data that looks like this:

\# Error:Cannot connect to database

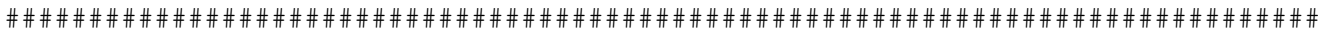

def pdb data is valid(pdb data):

pdb_data_stēing $=$ pdb_data.decode ("utf-8")

if $\bar{p}$ db data string $[: 6 \overline{]}==$ "HEADER":

else: return True

return False

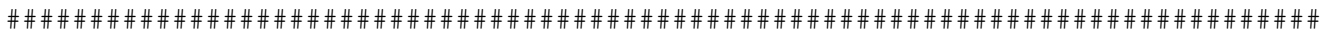
\# Get a list of pdb names from a file.

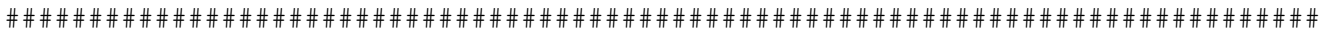

def get_pdb_list():

if $\bar{l}$ en (sys.argv) $!=2$ :

print "To run this script do the following: \n"

" \tpython download pdb.py pdb names.txt \n"

"\tor python download pdb.py $\overline{1} 19 \mathrm{LA} "$

sys.exit $(-1)$

pdb_list $=[]$

if os.path.exists (sys.argv[1]):

input_file_handle =open (sys.argv[1], "r") 


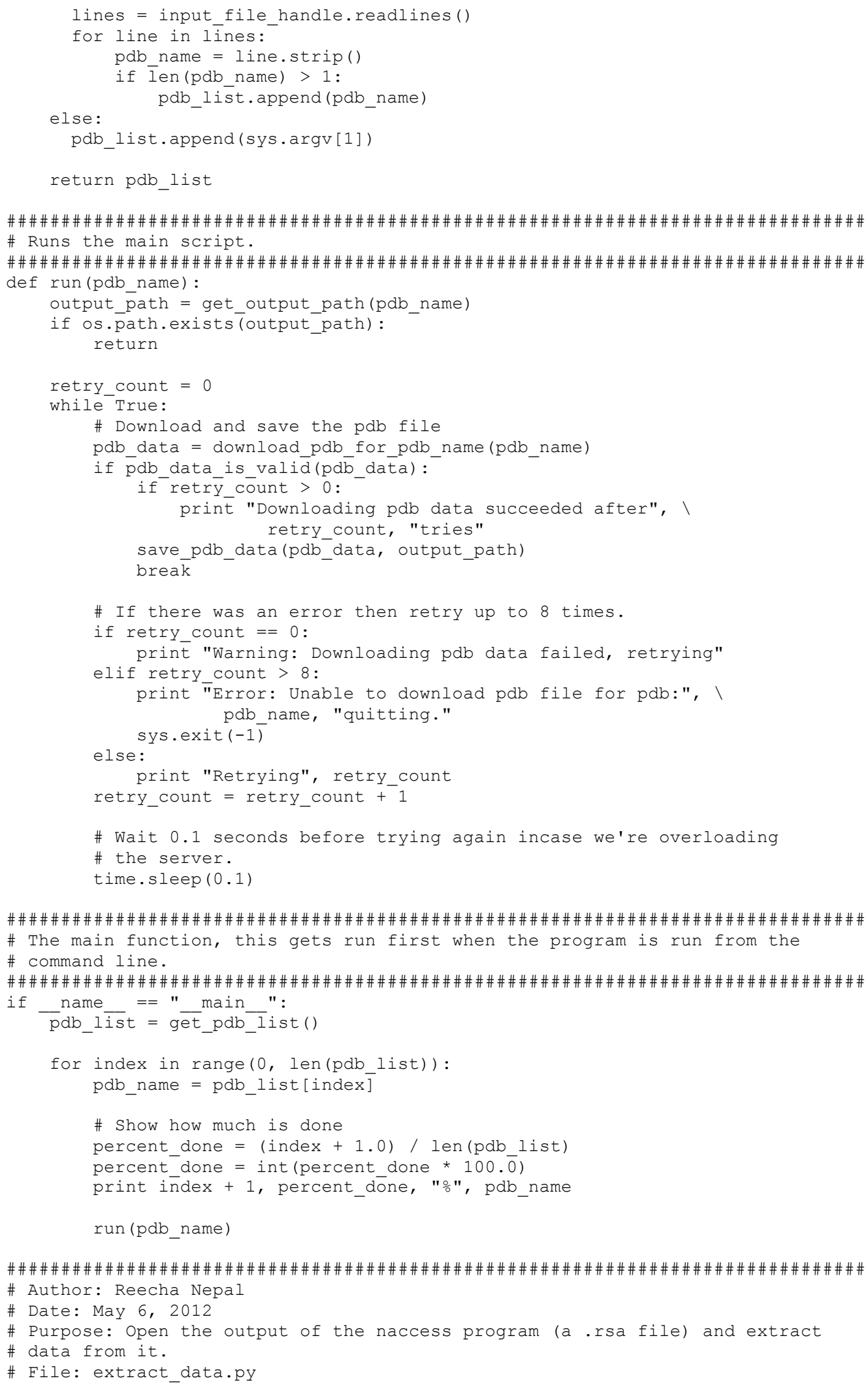




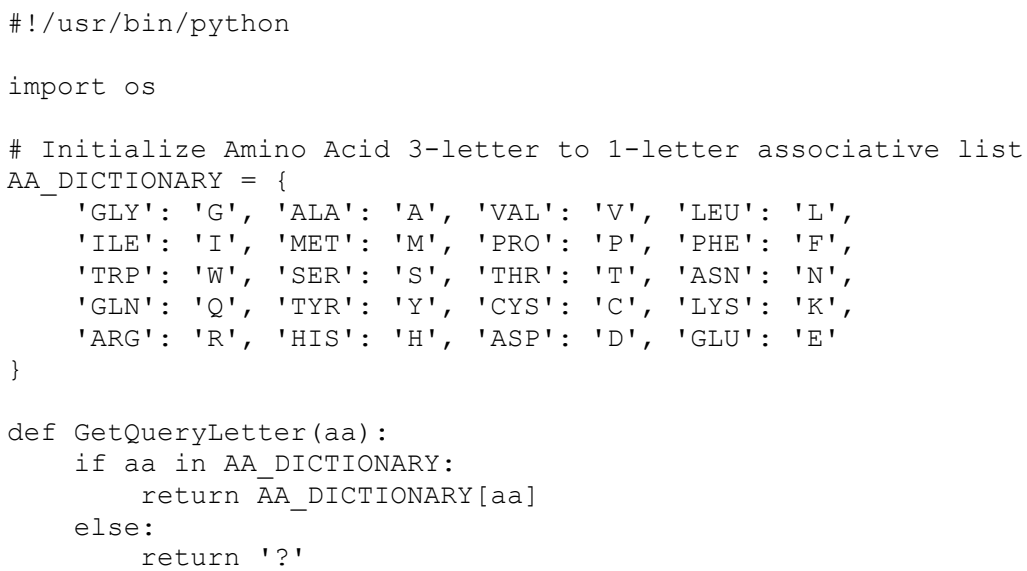

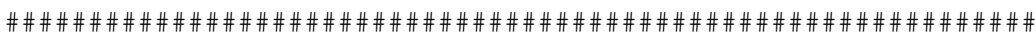




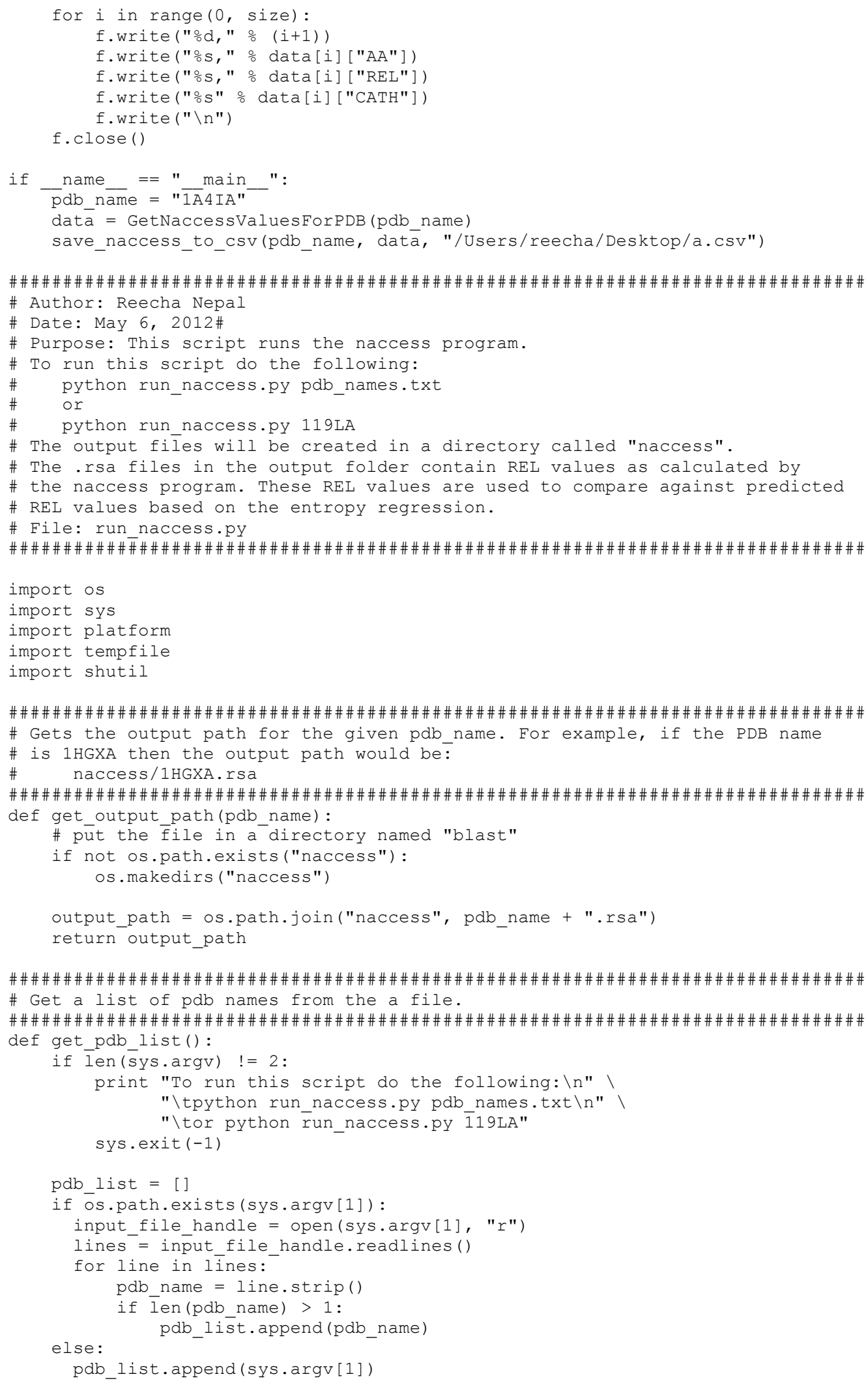


return pdb_list

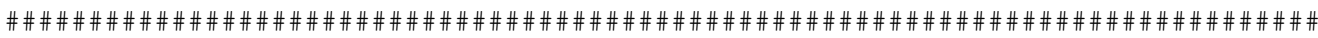

\# Gets the path to folder that contains the naccess code.

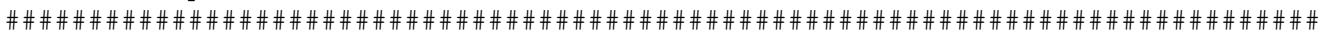

def get naccess code path():

script_path ${ }^{-}=$os.path.realpath (_file_)

parent_directory = os.path.dirname (script path)

return os.path.join (parent_directory, "NACCESS_Code")

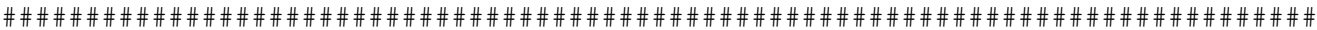

\# Gets the path the pdb file.

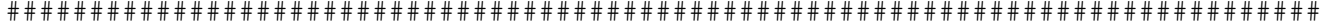

def get_pdb_file_path (pdb_name) :

pdb file path = os.path.join ("pdb", pdb name + ".pdb")

if not os.path.exists (pdb_file_path):

print "Couldn't find pdb file: ", pdb file path

print "Run the download_pdb.py script to download the pdb file first." sys.exit $(-1)$

return os.path.abspath (pdb_file_path)

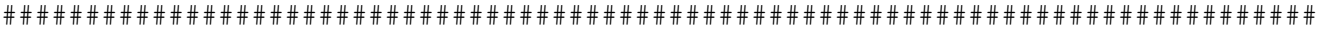
\# Runs the naccess program

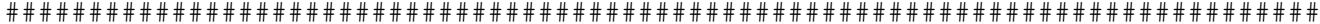

def run_naccess (pdb_name, pdb_file_path, naccess_code_path):

old working diréctory $=$ os $\cdot$ get $\bar{c} w d()$

temp_dir $=$ tempfile.mkdtemp ()

os.chdir(temp dir)

if platform.system() == "Windows":

naccess_program = os.path.join(naccess_code_path, "naccess_win.exe") else:

naccess program = os.path.join (naccess code path, "naccess")

vdw file path = os.path.join (naccess code_path, "vdw.radii")

os.system(naccess_program + " " + pdb_file_path + " -r " + vdw_file_path)

file name $=$ pdb name + '.rsa'

shutil.copy (filēename, os.path.join(old_working_directory, 'naccess'))

os.chdir (old working directory)

shutil.rmtree (temp_dir, ignore_errors=True)

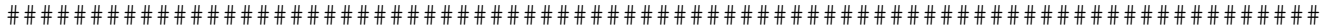

\# Runs the main script.

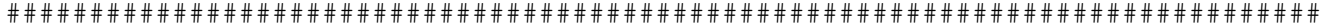

def run (pdb_name, naccess_code_path=None):

if not naccess code path:

naccess_code_path = get_naccess_code_path ()

os.environ["NACCESS_EXE_PATH"] = nacCess_code_path

output_path = get_output_path (pdb_name)

if os.path.exists (output_path):

return

pdb_file_path = get_pdb_file_path (pdb_name)

run naccéss (pdb_name, pōb_file_path, naccess_code path)

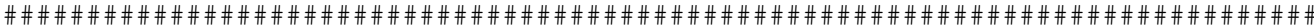

\# The main function, this gets run first when the program is run from the

\# command line.

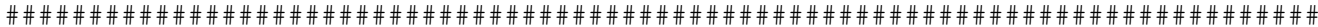

if name___ = " main ":

naccess_code_path $=$ get_naccess_code_path ()

pdb_list $=$ get_pdb_list ()

for index in range (0, len(pdb_list)) :

pdb name $=$ pdb list $[$ index $]$ 


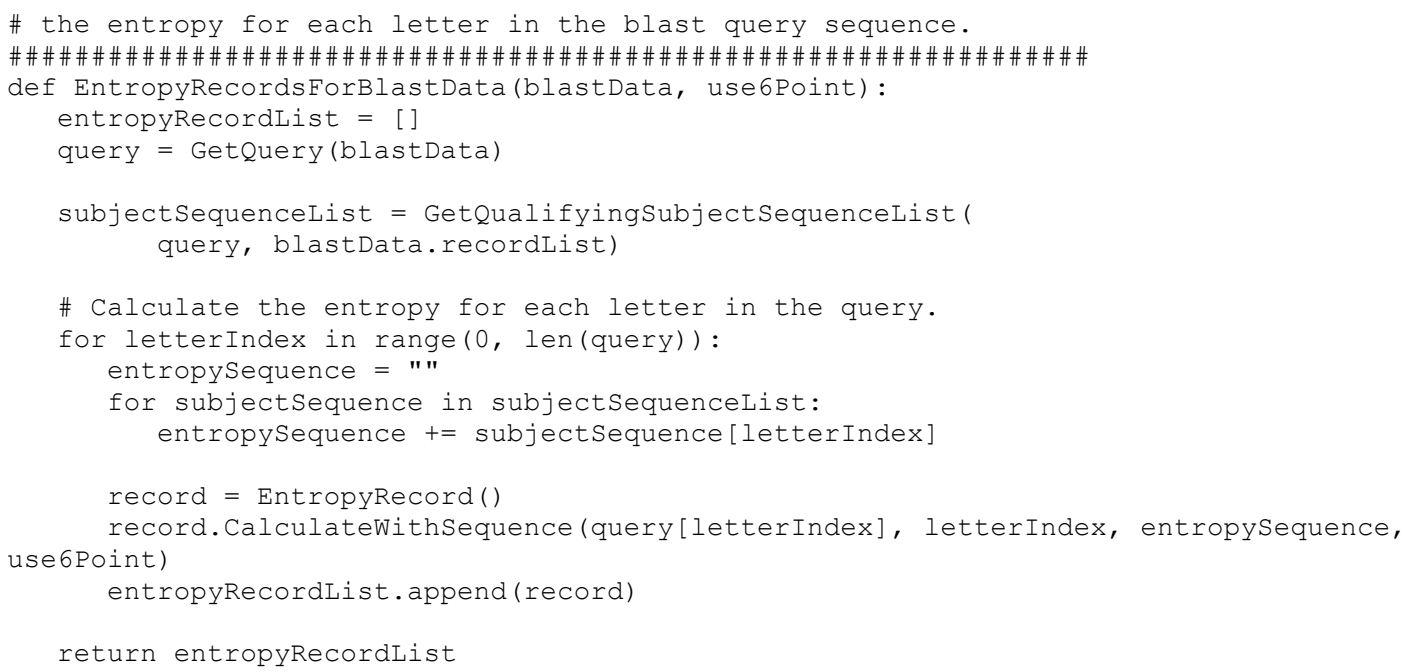




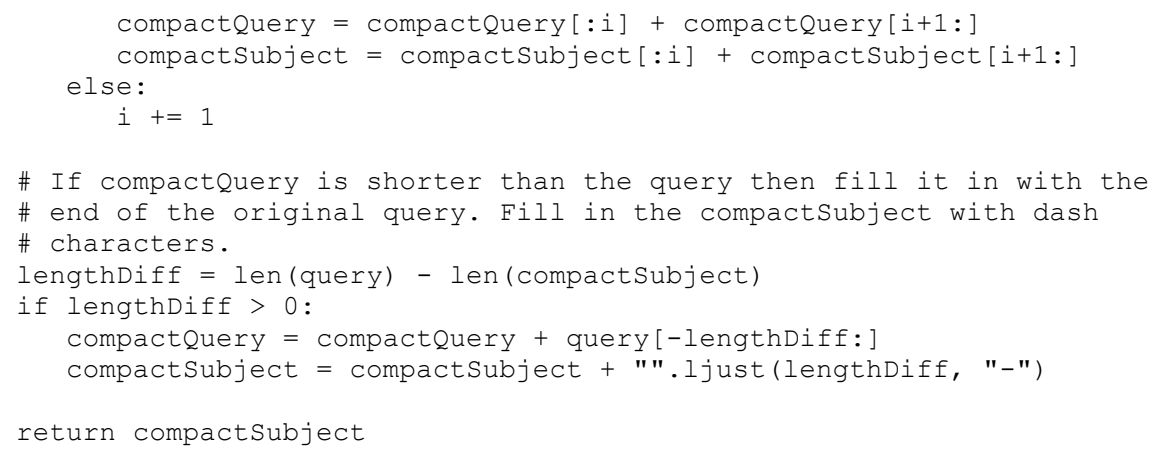




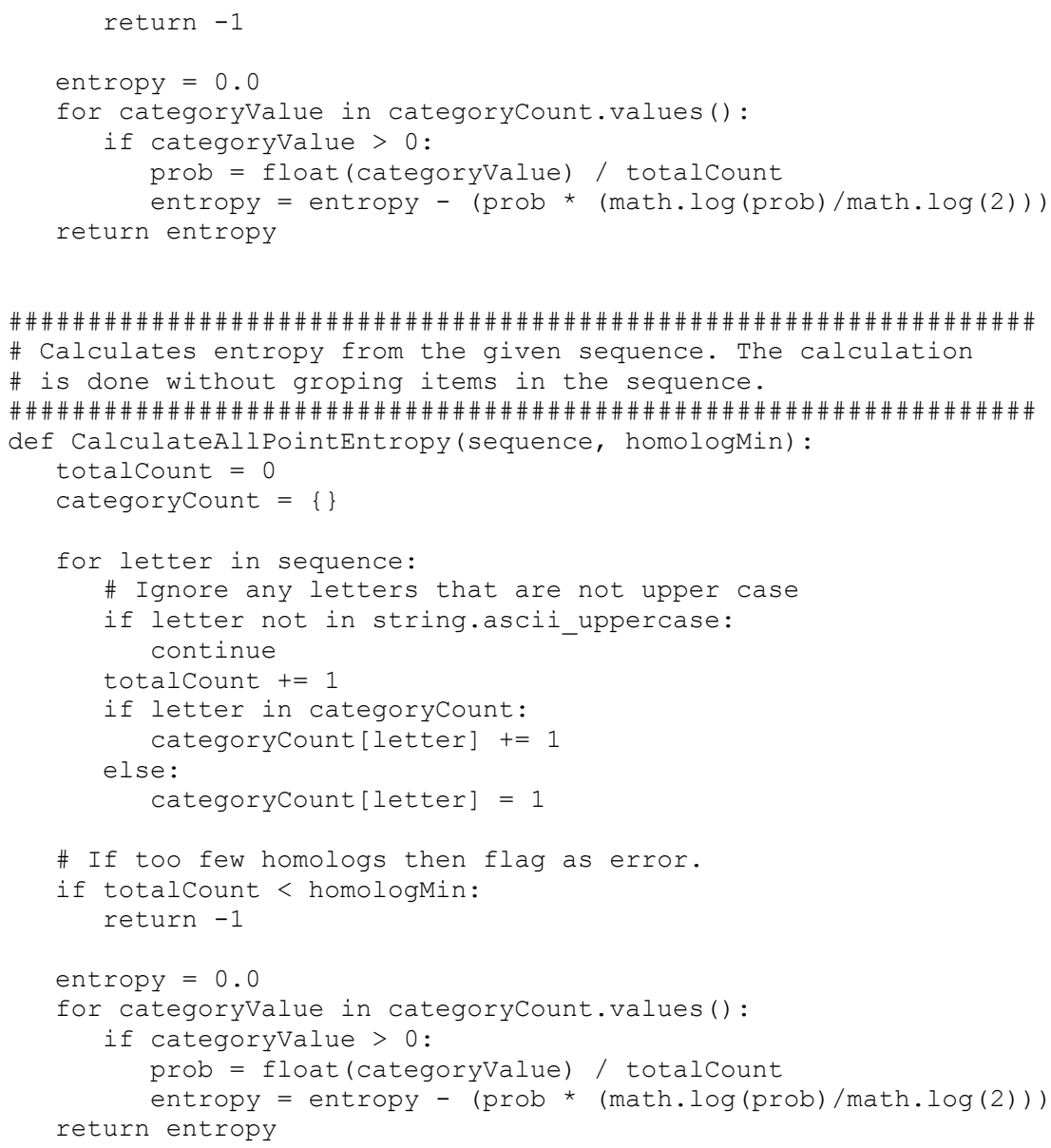

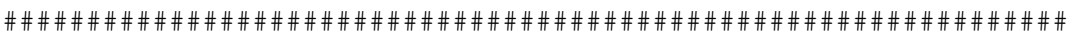

\# Normally this script is not run directly. Callers should just

\# use the EntropyRecordsForBlastData function to get the 
out_file.close()

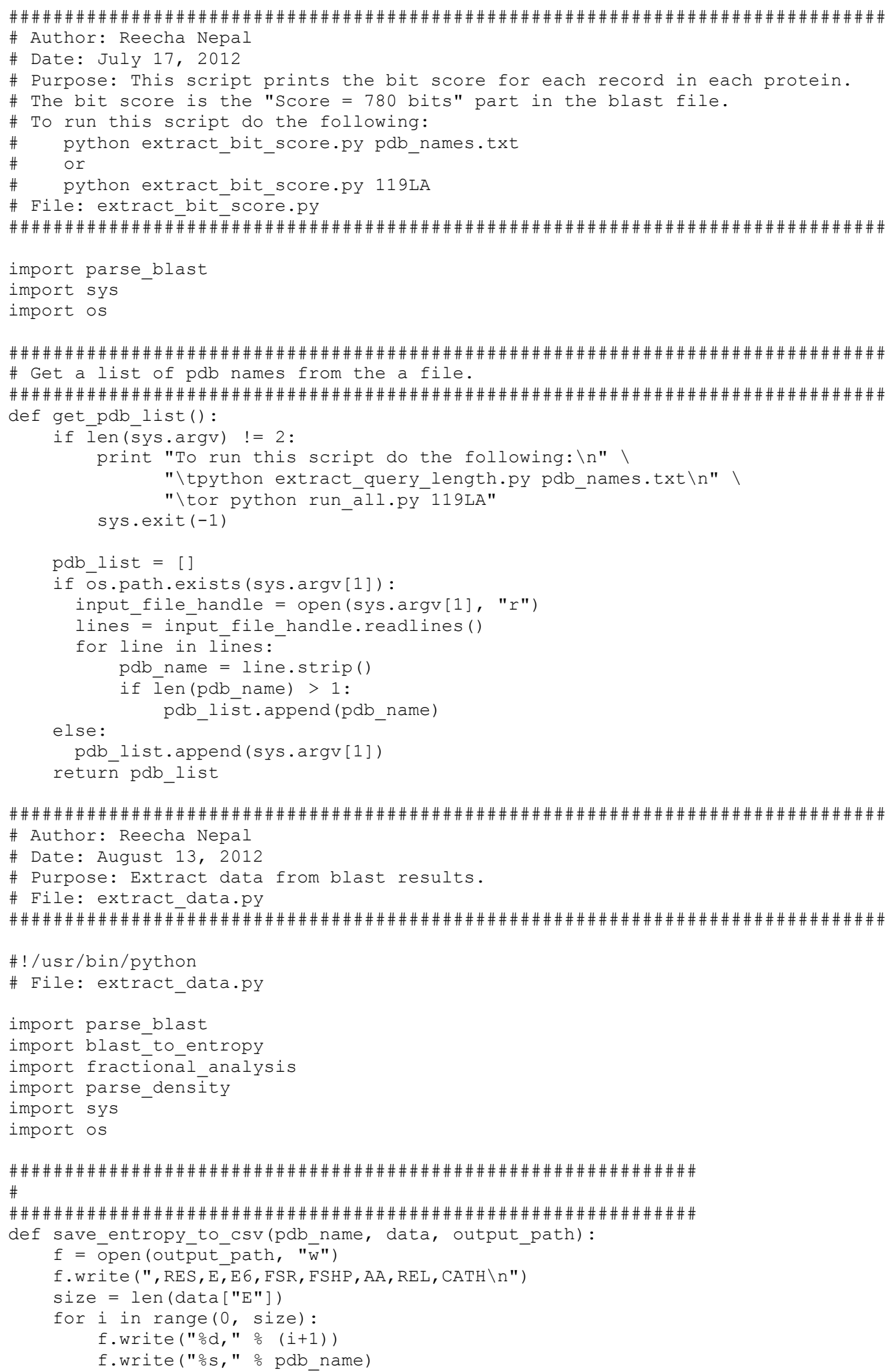




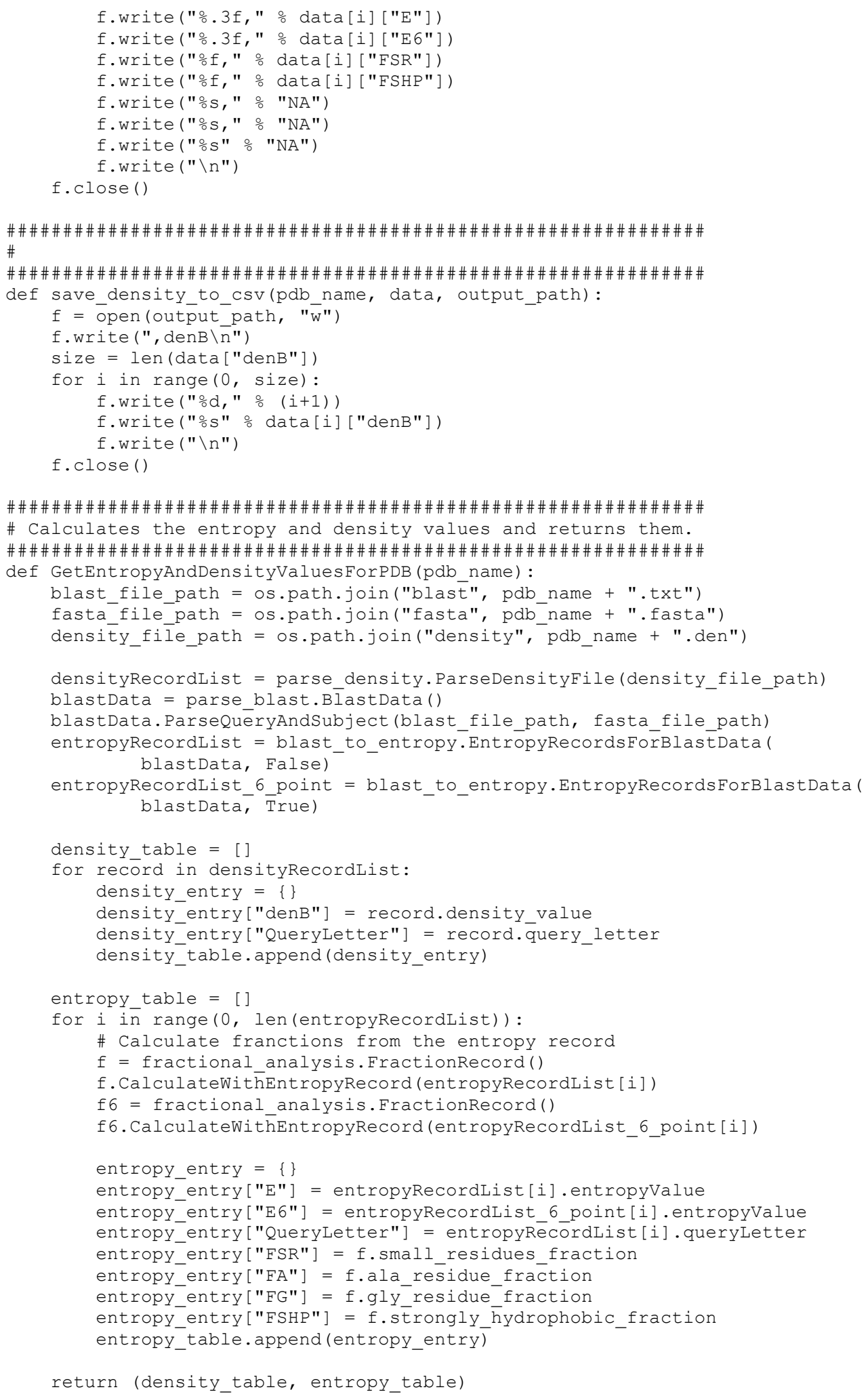

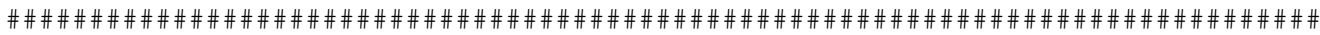




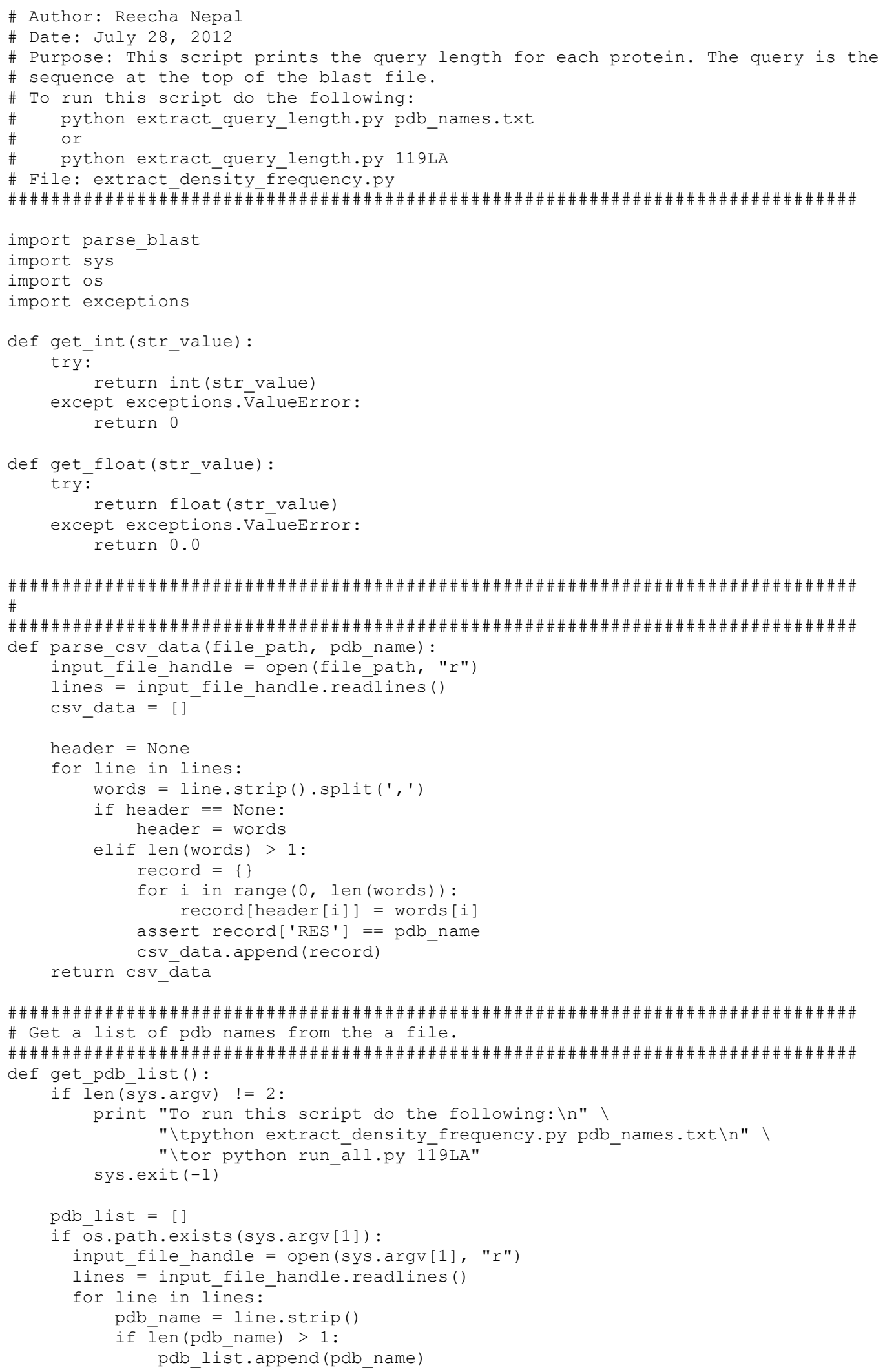




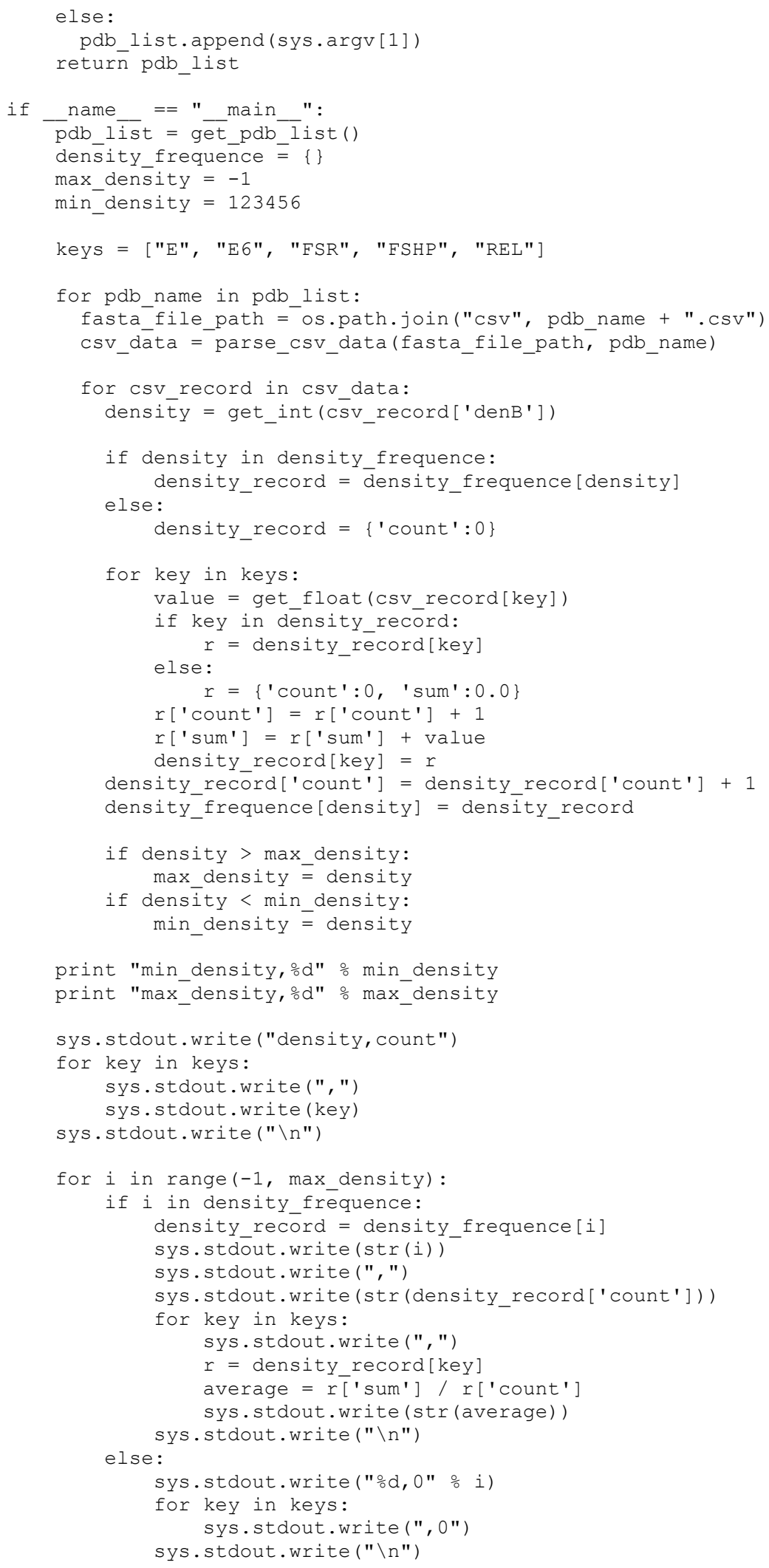




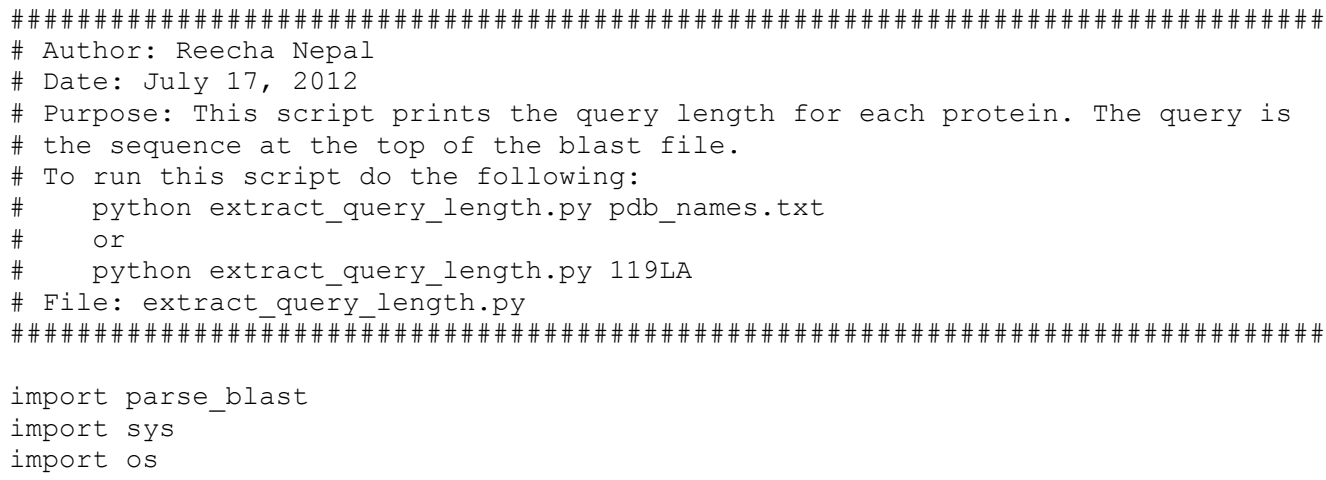




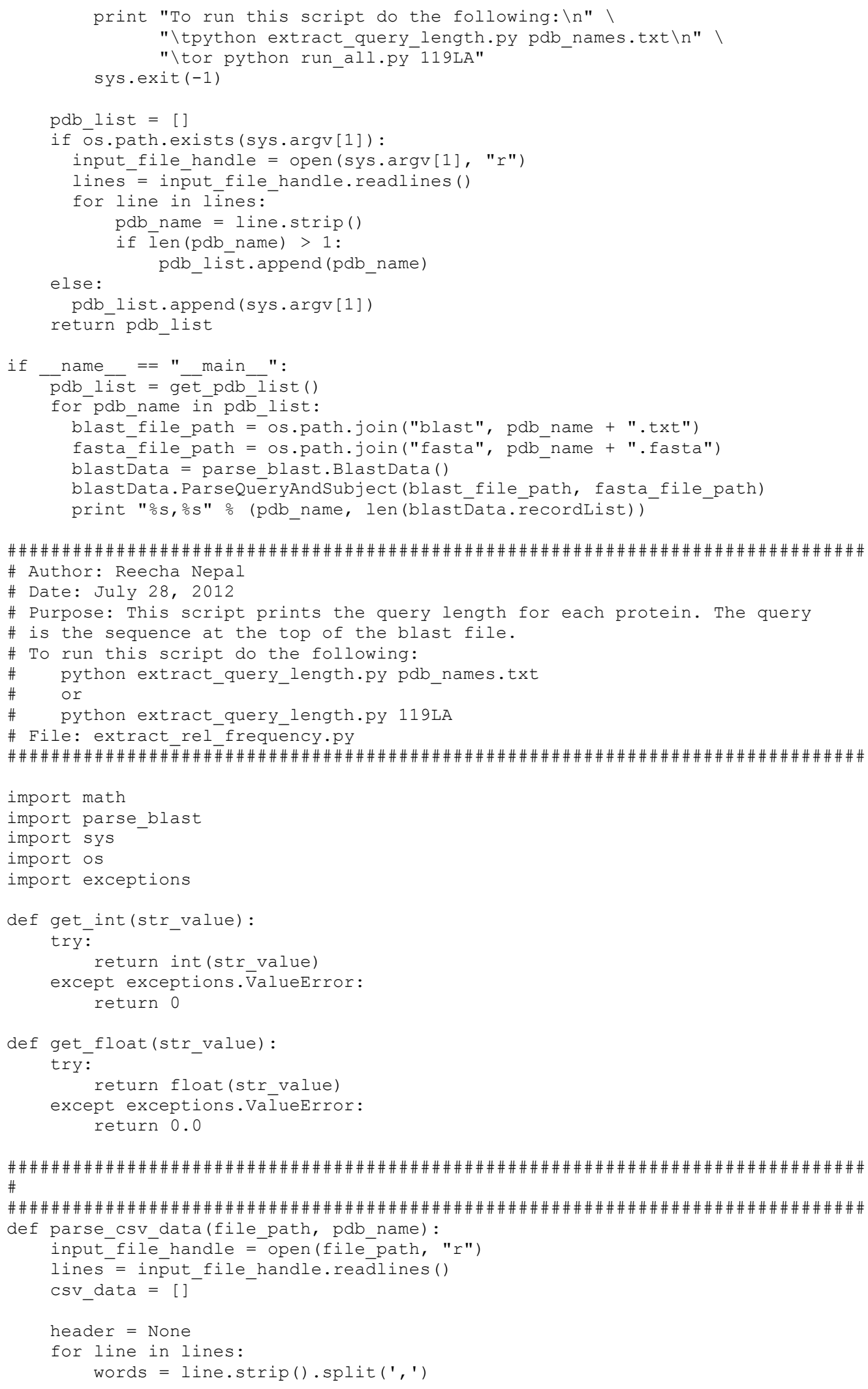




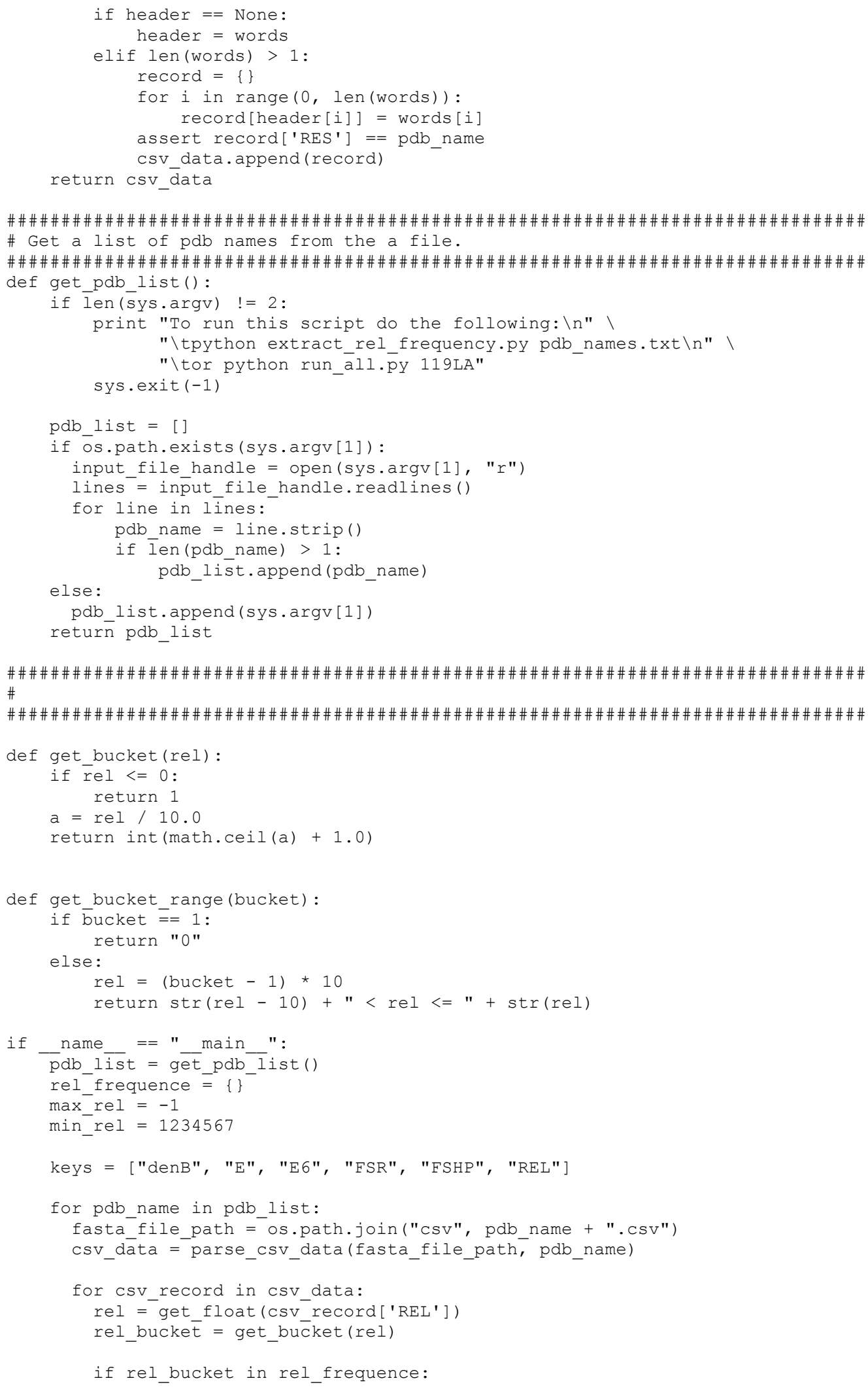




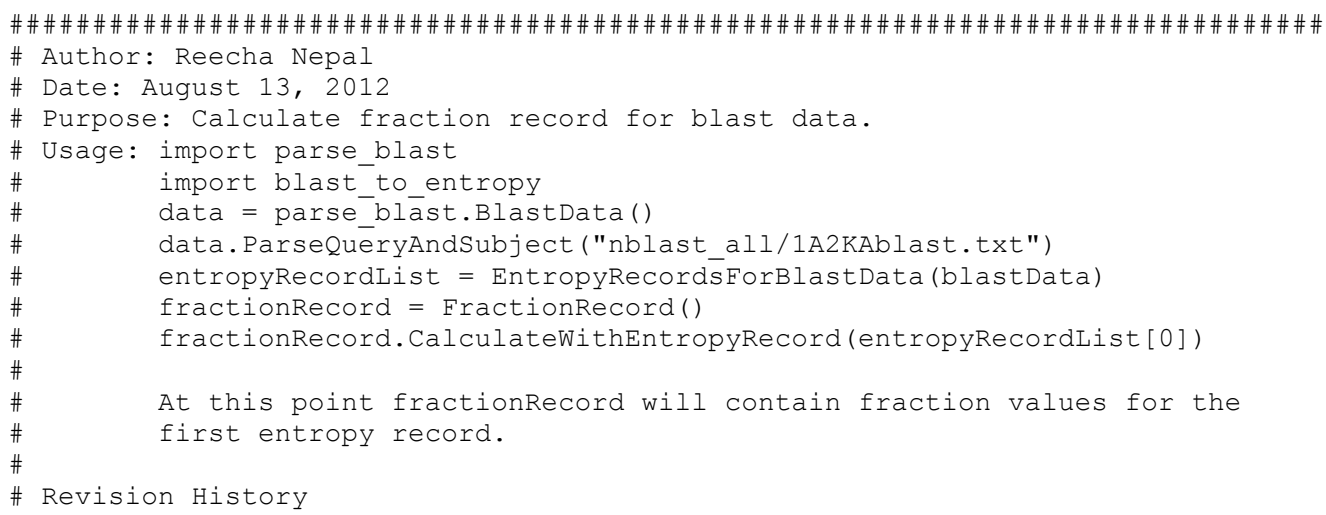




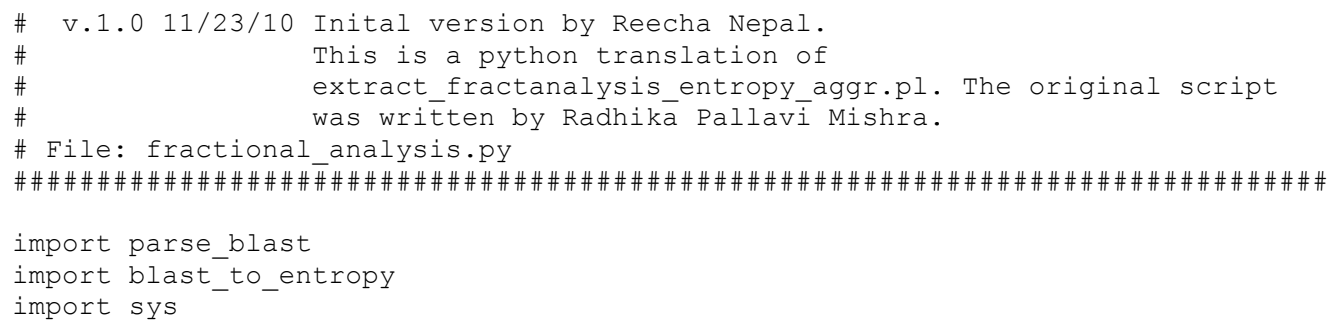

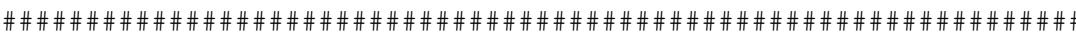

\# Stores fractions computed from a single entropy record.

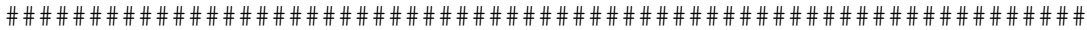

class FractionRecord:

def init (self):

self.gap fraction $=0.0$

self. small residues fraction $=0.0$

self.ala residue fraction $=0.0$

self.gly residue fraction $=0.0$

self.strongly hydrophobic fraction $=0.0$

self.non_strongly_hydrophöbic_fraction $=0.0$

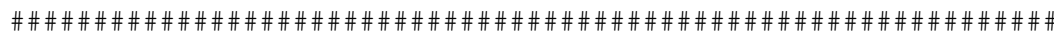

\# Fills in the FractionRecord object using fractions computed

\# from the given entropy record.

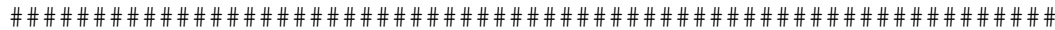

def CalculateWithEntropyRecord(self, entropyRecord):

gap count $=0$

small residues count $=0$

ala_résidue count $=0$

gly residue count $=0$

strongly_hyōrophobic_count $=0$

total length = len (entropyRecord.entropySequence)

for letter in entropyRecord.entropysequence:

if letter in "-":

gap_count $+=1$

elif letter in "AG":

small residues count $+=1$

if lēter $==" \overline{\mathrm{A}} "$ :

else:

ala_residue_count $+=1$

gly residue count $+=1$

elif letter in "VIT̄FYMW":

strongly hydrophobic count $+=1$

num non gap amino acids = total length - gap count

if num non_gap_amino_acids > 0 :

seléf.gap fräction ${ }^{-}=$float (gap_count) / num non gap amino acids

self.small_residues_fraction = float(small_residues_count) /

num non gap amino acids

self.ala residue fraction = float (ala residue count) / num non gap amino acids self.gly_residue_fraction = float (gly_residue_count) / num_non_gap_amino_acids

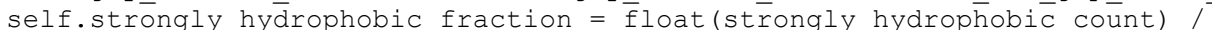

num_non_gap_amino_acids

self.non strongly hydrophobic fraction $=1.0-$

self.strongly_hydrophobic_féraction

else:

self.gap fraction $=0.0$

self.small residues fraction $=0.0$

self.ala residue fraction $=0.0$

self.gly residue fraction $=0.0$

self.strongly hydrophobic fraction $=0.0$

self.non_strongly_hydrophöbic_fraction $=0.0$ 


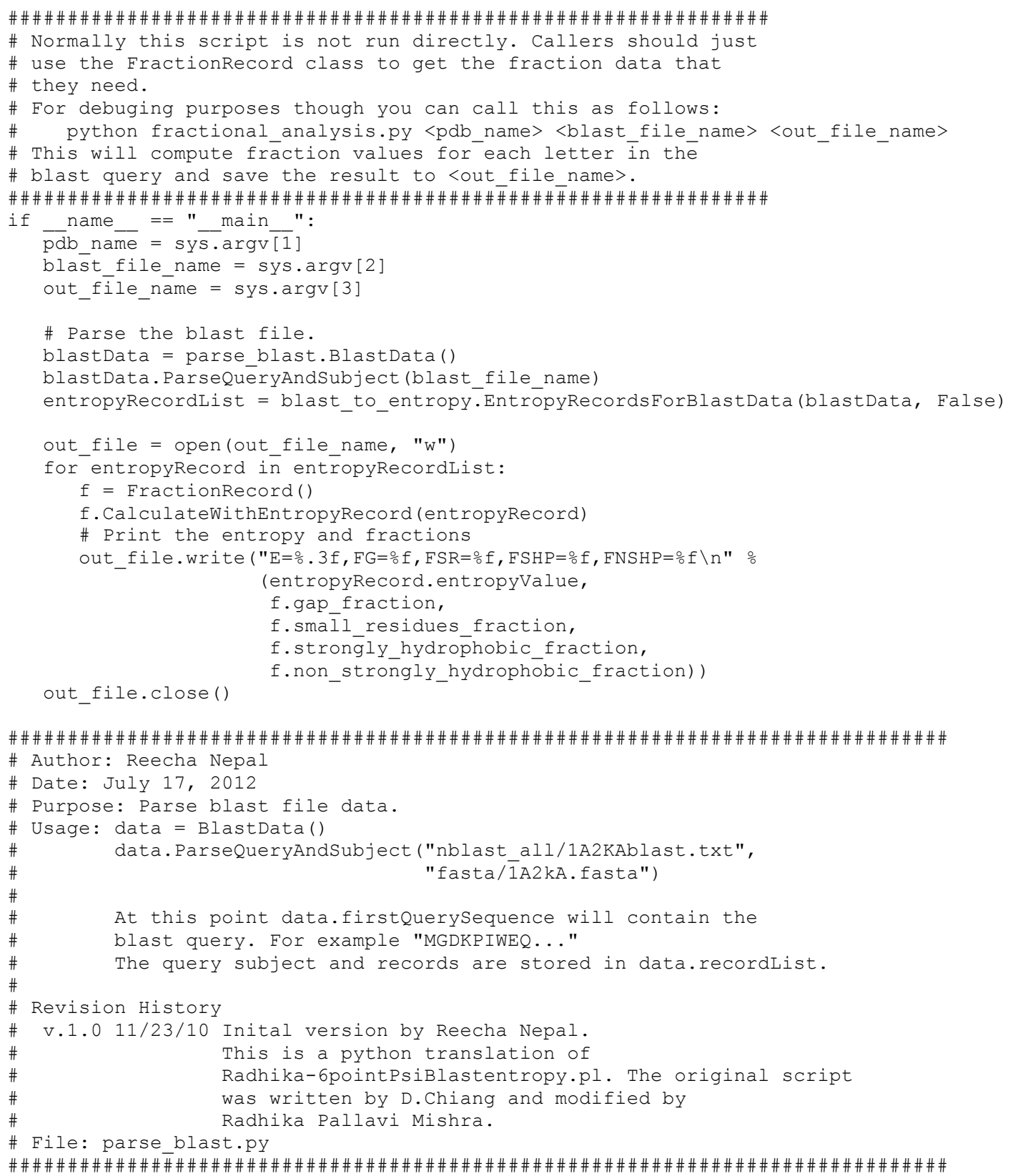

import sys

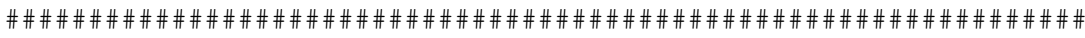

\# Stores a single query and subject record.

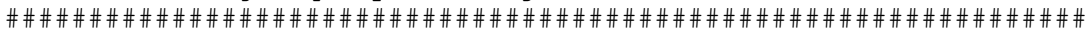

class QueryAndSubjectRecord:

def _init__(self) :

sélf.scoreBits $=0.0$

self.percentIdentities $=0.0$

self.percentPositives $=0.0$

self.expectValue $=0$

self.querysequence $=" "$

self.subjectSequence = " "

self.queryoffset $=0$ 
self.lineStartIndex $=0$

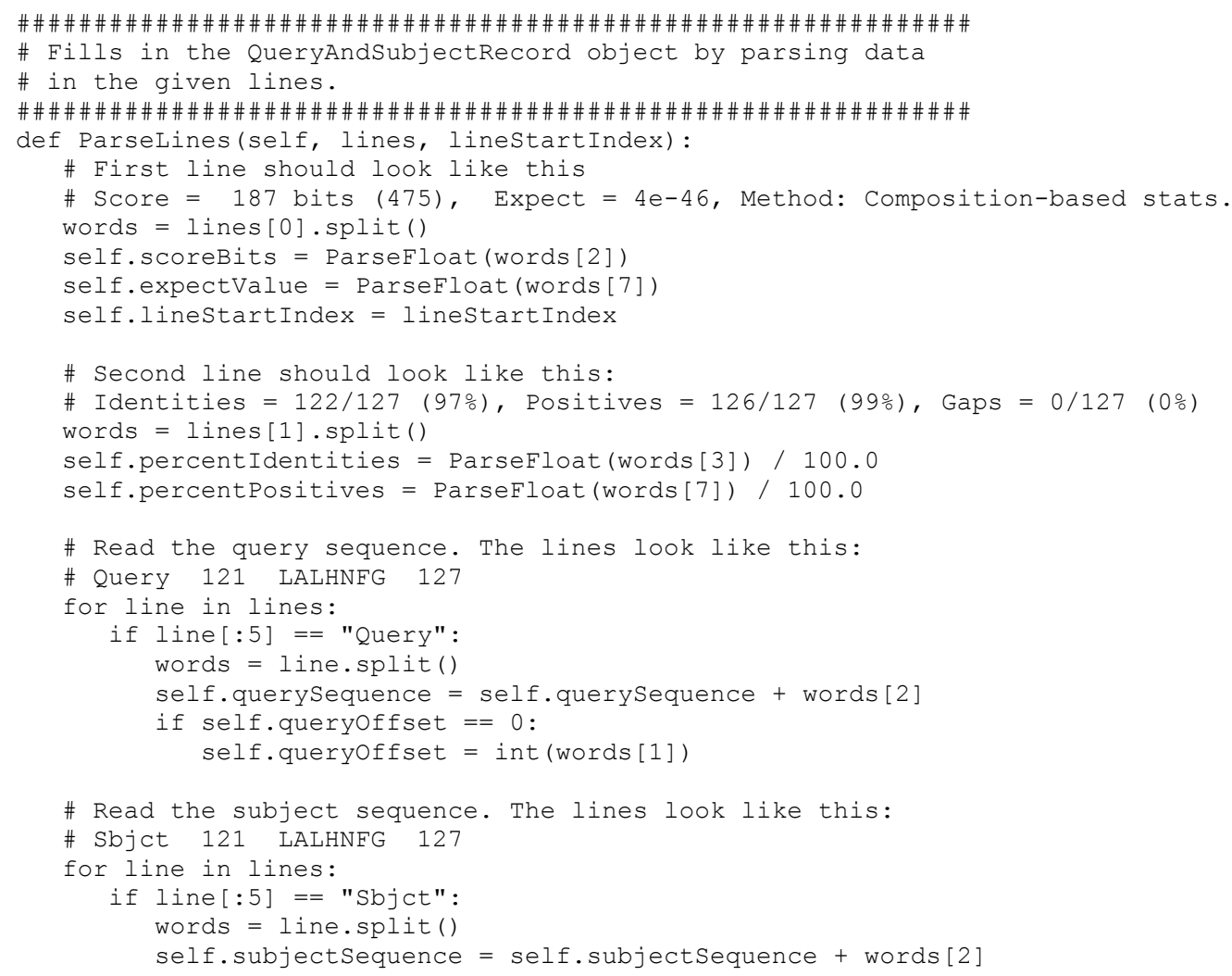

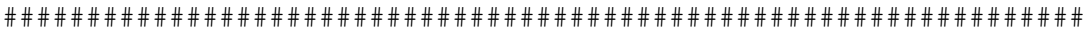

\# This class stores data from the results of a blast query.

\# It stores the original query and a list of query and subject

\# records.

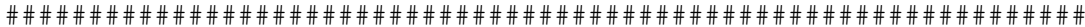

class BlastData:

def init (self):

sēlf.recordList $=[]$

self.firstQuerysequence = " "

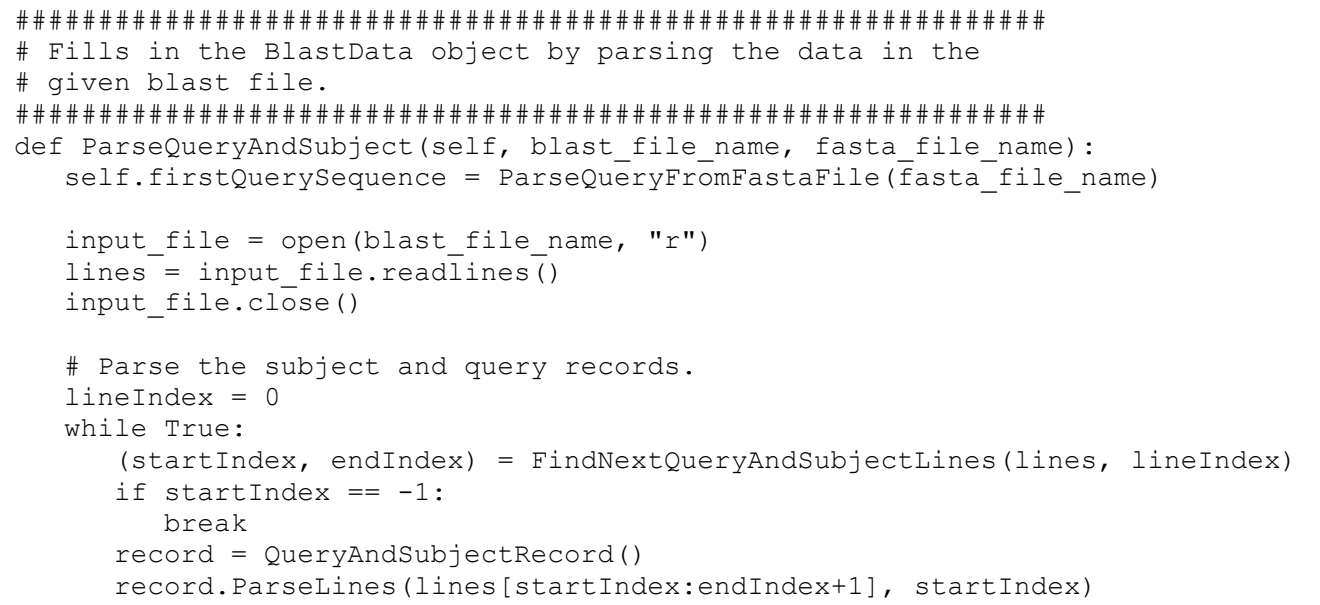


self.recordList. append (record)

lineIndex $=$ endIndex +1

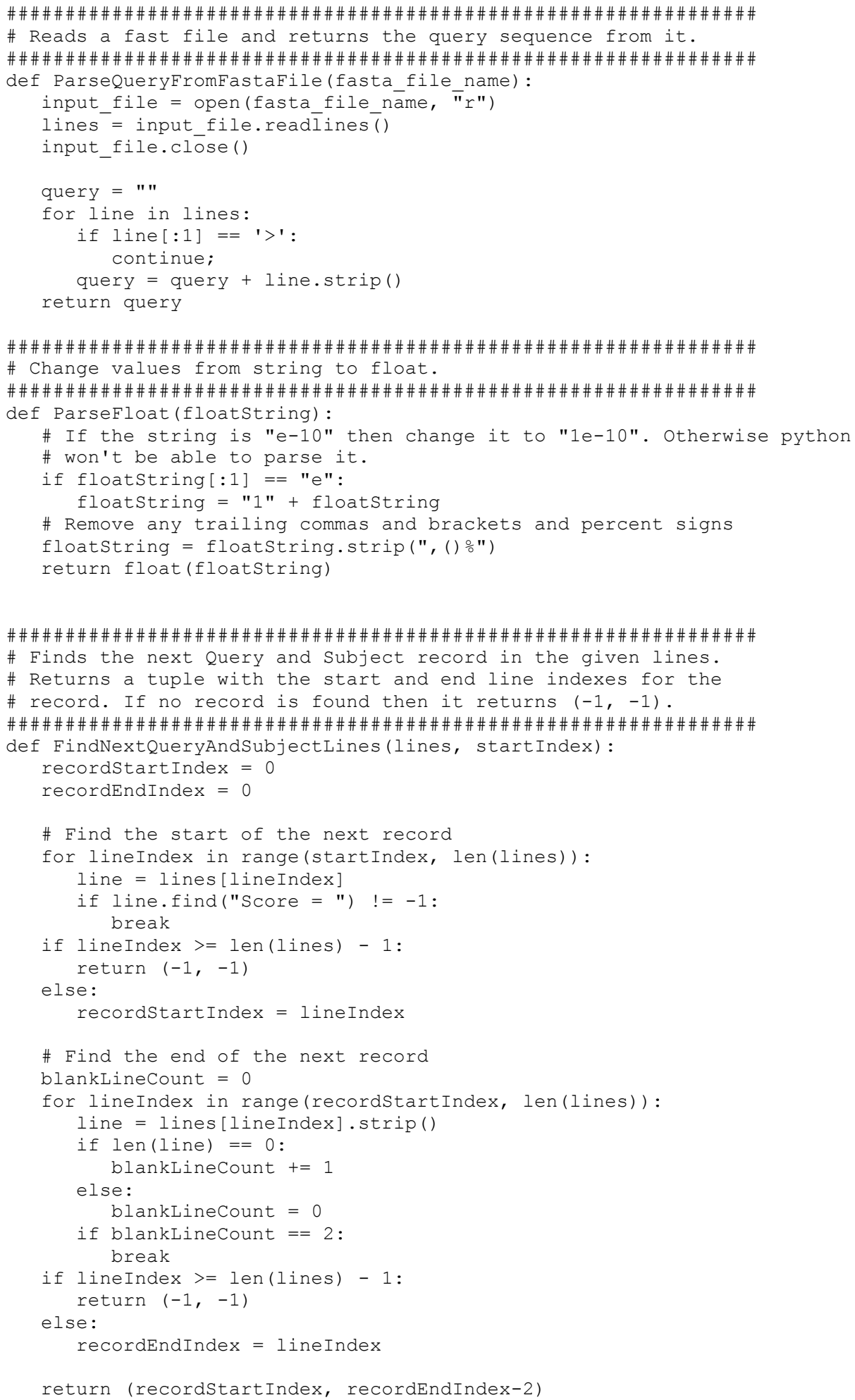




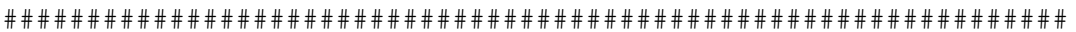

\# Normally this script is not run directly. Callers should just

\# use the BlastData object to get the data they need.

\# For debuging purposes though you can call this as follows:

\# python parse blast.py <blast file name> <fasta file name>

\# This will print the first query sequence, the first record,

\# and the total number of records.

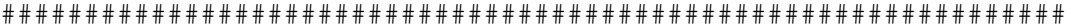

if name $==$ "main " :

blast file name $=$ sys.argv[1]

fasta_file_name = sys.argv[2]

data $=$ BlastData ()

data.ParseQueryAndSubject(blast file name, fasta file name)

print "First query sequence is", datā.firstQuerysequeñce

print "found", len(data.recordList), "records"

record = data.recordList [0]

print "The first record is"

print "scoreBits", record.scoreBits

print "percentIdentities", record.percentIdentities

print "percentPositives", record.percentPositives

print "expectValue", record.expectValue

print "querysequence", record.querysequence

print "subjectsequence", record.subjectsequence

print "queryoffset", record.queryoffset

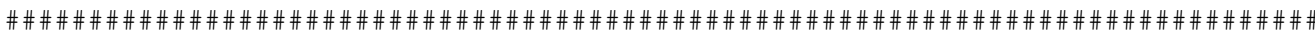

\# Author: Reecha Nepal

\# Date: May 6, 2012

\# "Parse Density"

\#

\# Purpose: Parse the density file.

\# Usage: import parse_density

densityRecordList = parse density.ParseDensityFile("1alia.den")

At this point densityRecordList will contain a list of density values.

Revision History

v.1.0 11/23/10 Inital version by Reecha Nepal.

This is a python translation of

svm_extract fractentropy_density_aggr.py. The original script was written by Radhika Pallavi Mishra.

\# File: parse_density.py

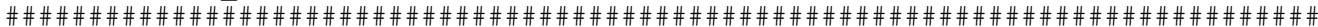

import sys

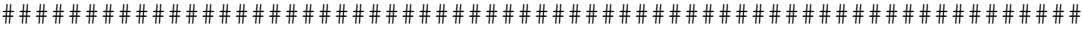

\# Stores a single density value and the pdb position for that

\# value.

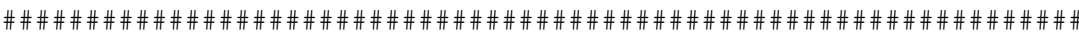

class DensityRecord:

def _init___ (self):

density value $=" "$

pdb_pos $="$ "

query letter $=" "$

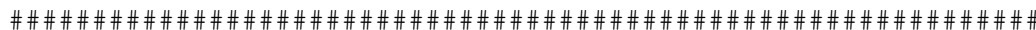

\# Fills in the DensityRecord object by parsing data in the

\# given line.

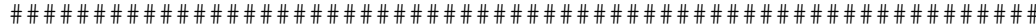

def Parseline(self, line):

words = line.split()

self.density_value = words [9].strip()

self.pdb pos $=$ words[16].strip()

query_words = words[1].split('_') 


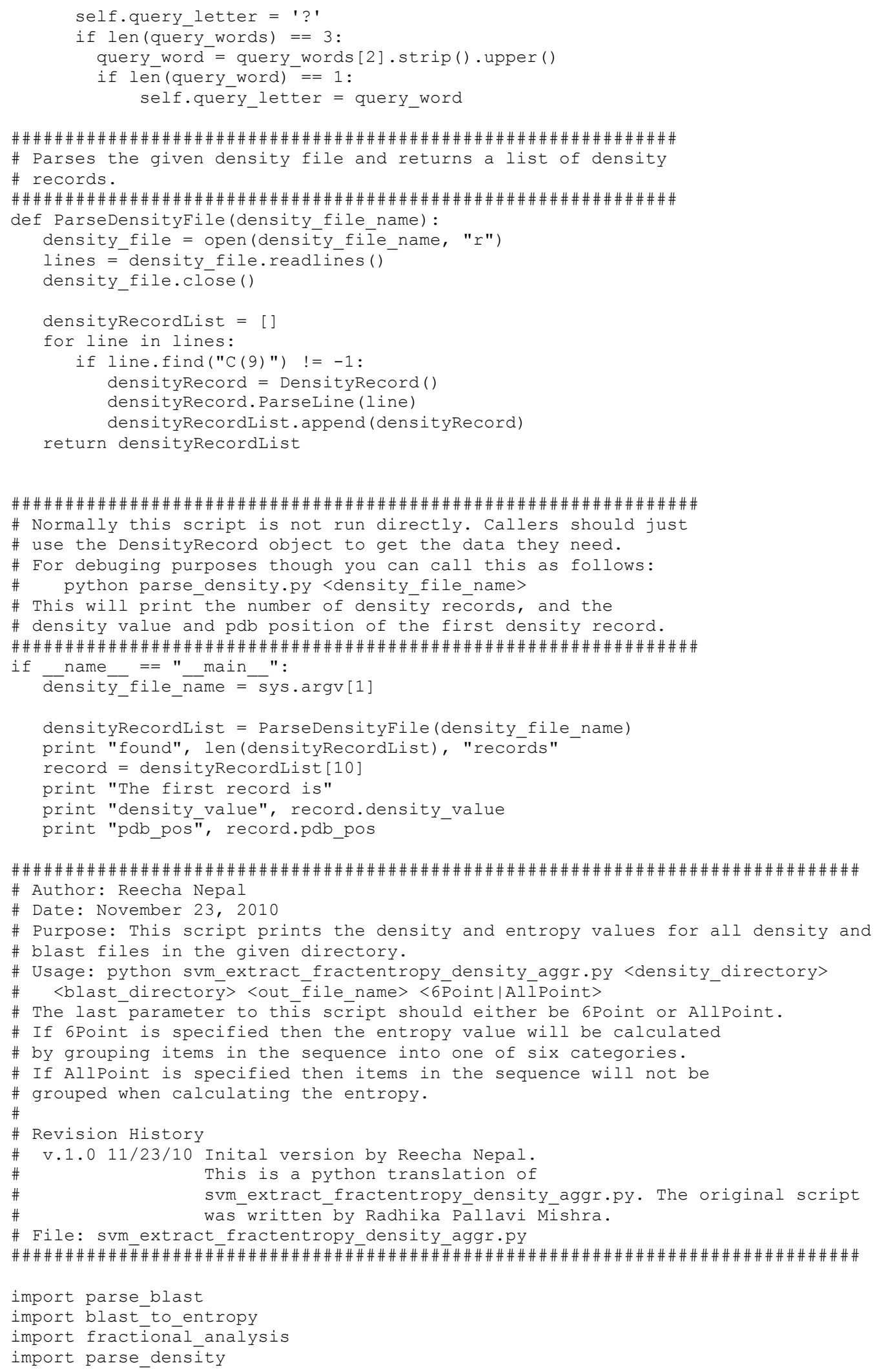


import sys

import os

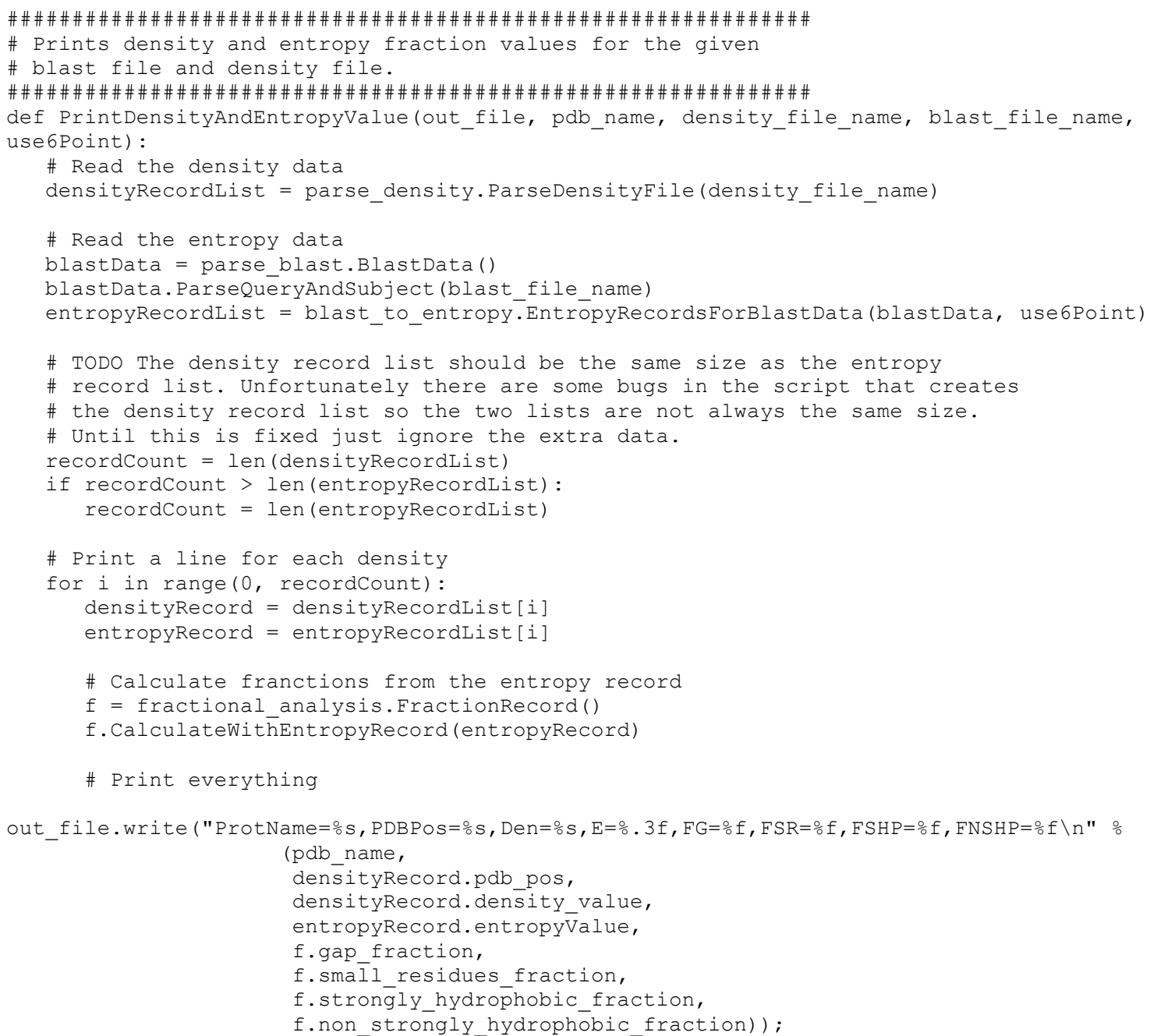




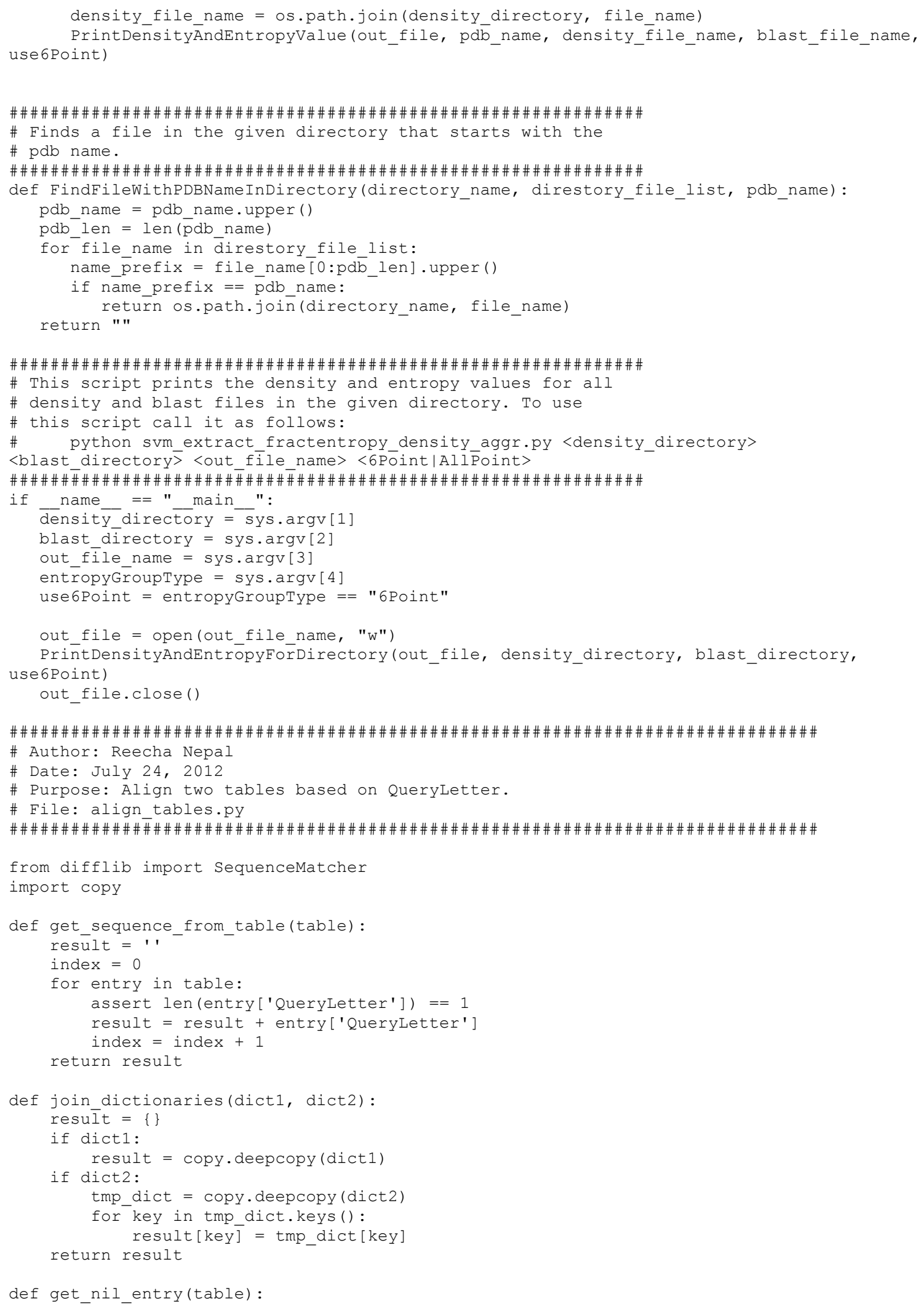




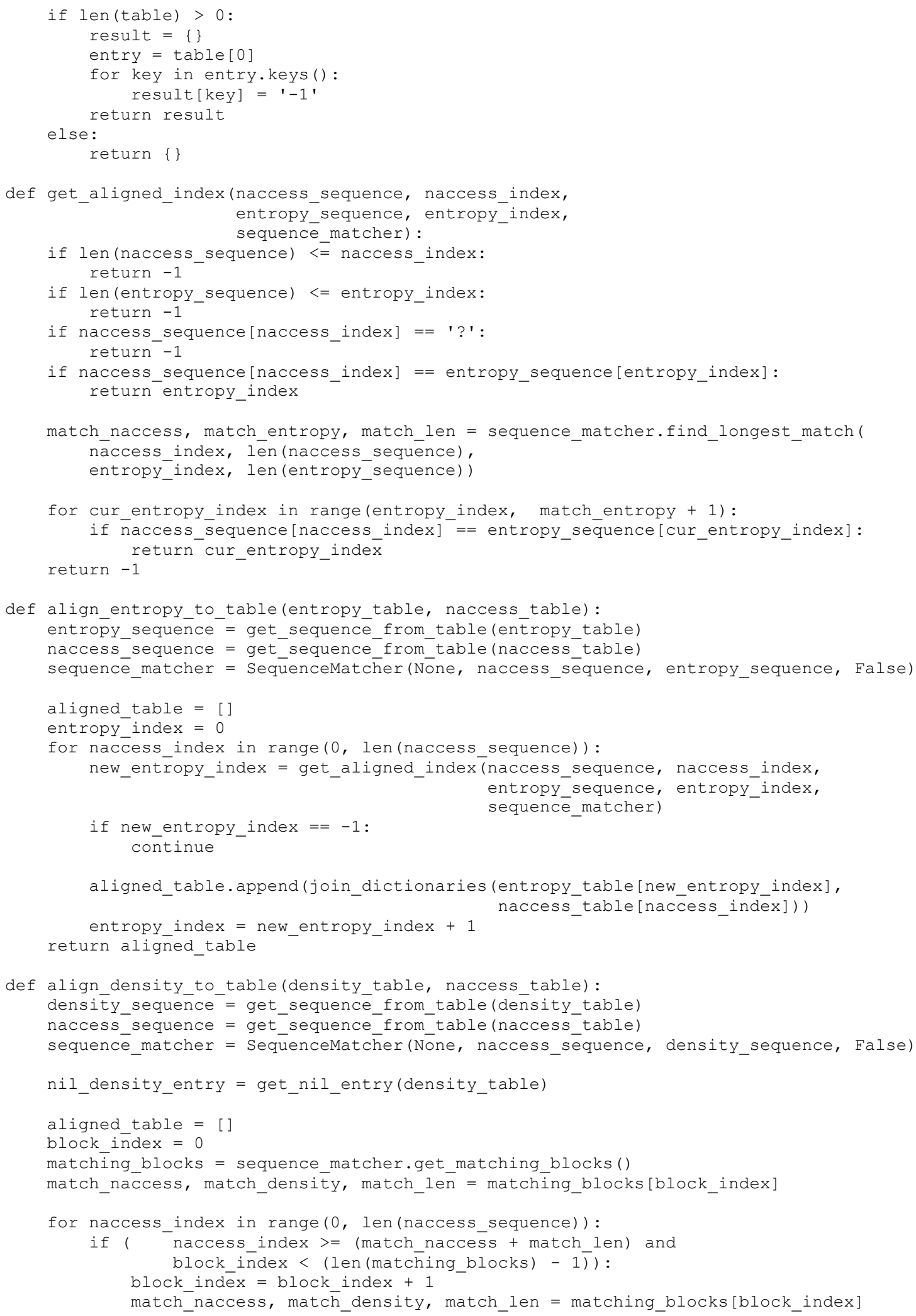




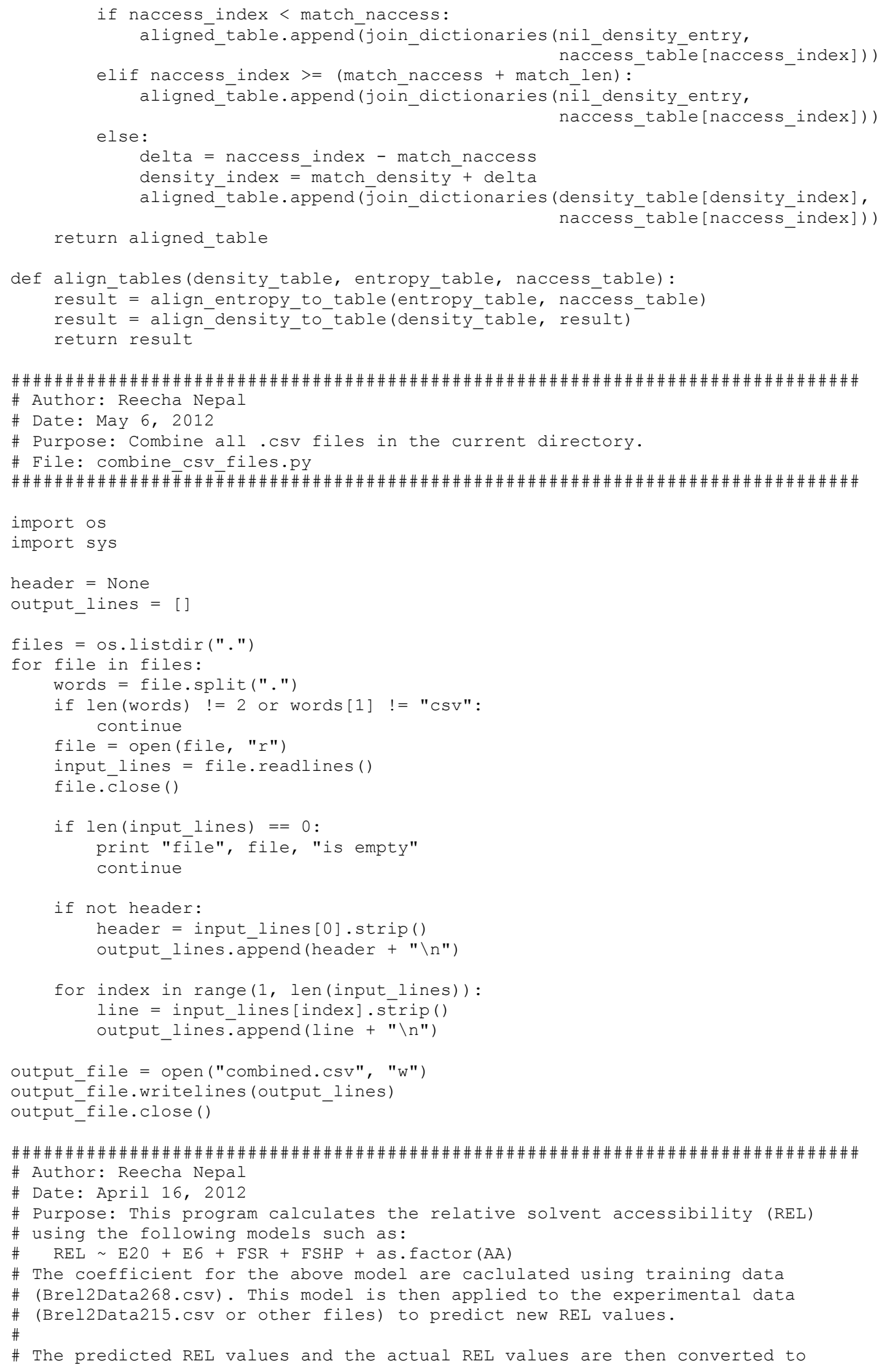




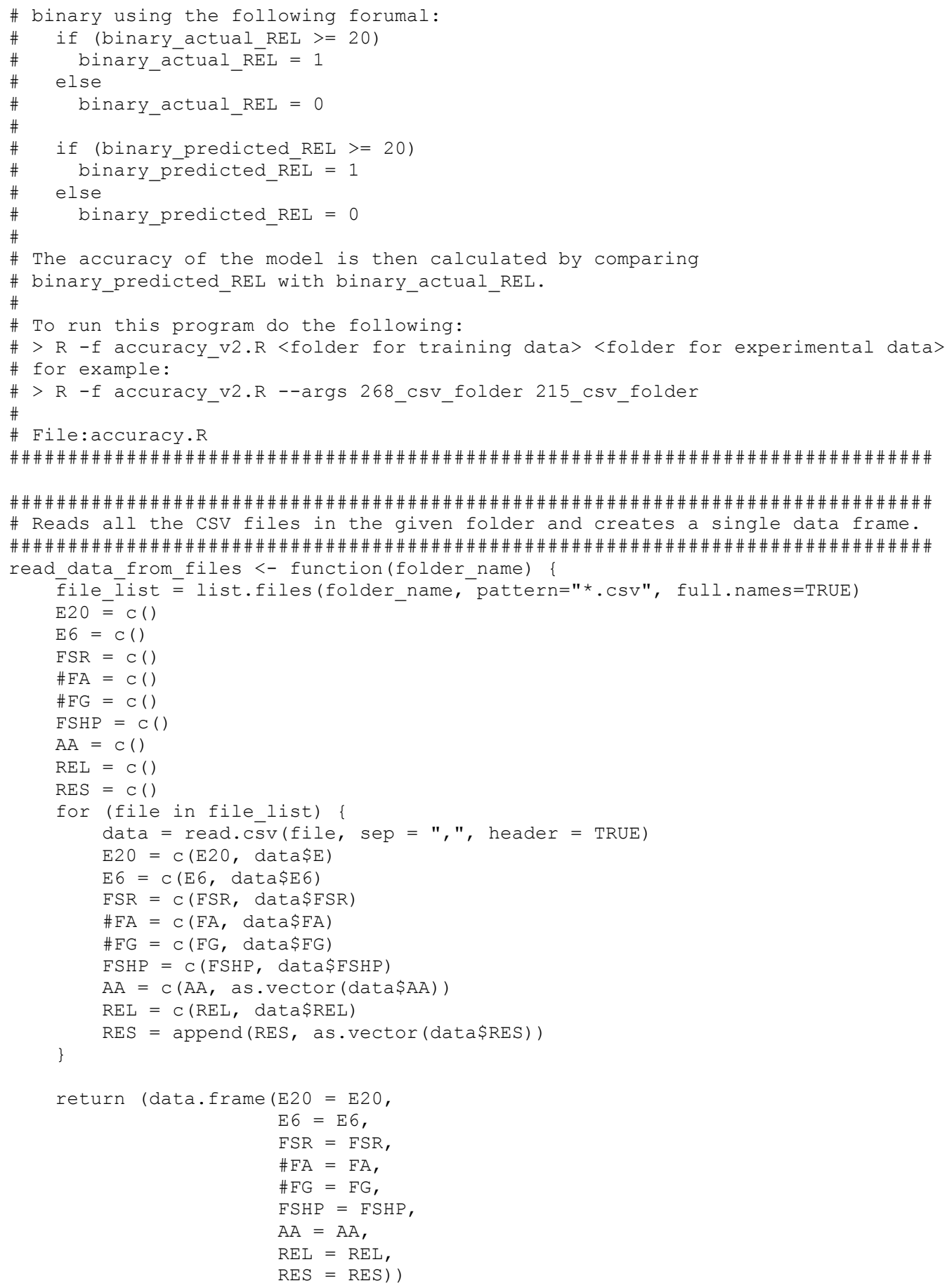




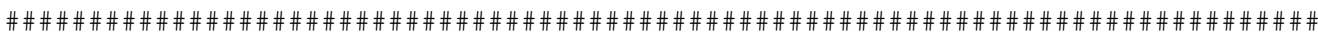
apply_model <- function(model, exp_data) \{

mätch_count $=0$

predicted values = predict (model, exp data)

for (i in 1:length (predicted_values))

actual REL = exp data\$REL̄ [i]

if (actual REL $>=20$ )

else actual_REL_Binary $=1$

actual_REL_Binary $=0$

predicted_REL = predicted_values $[i]$

if (predicted_REL > 23) \# 25.2

else predicted_REL_Binary $=1$

predicted_REL_Binary $=0$

if (actual_REL_Binary $==$ predicted_REL_Binary)

\} match_count $=$ match_count +1

\}

return (match_count)

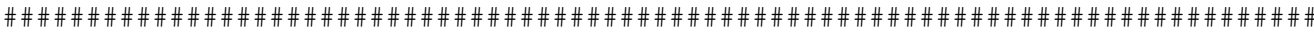
\# Save the predicted values in a CVS file for each PDB name.

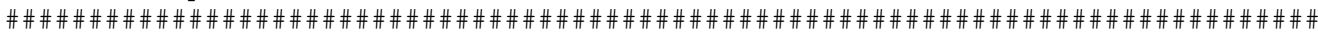

save predicted values <- function(i, model, exp data) \{

folder_name = paste ("predicted_", i, sep="")

dir.crēate(folder_name, showWarnings=FALSE)

predicted_values $=$ predict (model, exp_data)

for (i in 1 :length (predicted values))

pdb_name = exp_data\$RES $[\bar{i}]$

\# remove any white space

pdb_name = gsub (" +", "", pdb_name)

file name = paste (folder name, "/", pdb name, ".txt", sep=" )

\}

cat (predicted_values[i], file=file_name, sep=" $\backslash \mathrm{n} "$, append=TRUE)

\}

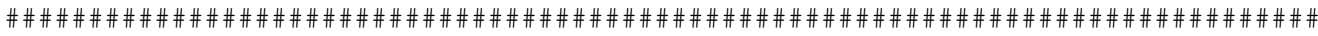

\# Read all the training csv files and setup models based on the data.

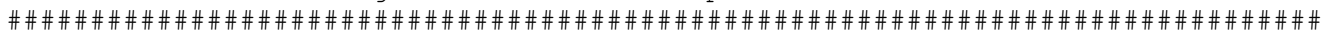
args $=$ commandArgs (trailingOnly = TRUE)

training_data = read_data_from_files(args[1])

models $=$ list ()

model_names $=\mathrm{C}()$

models $[[1]]=\operatorname{lm}($ REL $\sim$ E20, training_data)

model names $[1]=$ "REL E20"

models $[[2]]=\operatorname{lm}(\mathrm{REL} \sim \mathrm{E} 6$, training data)

model_names [2] = "REL $\sim \mathrm{E} 6$ "

models $[[3]]=\operatorname{lm}(\mathrm{REL} \sim \mathrm{FSHP}$, training data)

model_names $[3]=$ "REL $\sim$ FSHP"

models $[[4]]=\operatorname{lm}(\mathrm{REL} \sim \mathrm{FSHP}+$ as.factor $(\mathrm{AA})$, training data)

model_names $[4]=$ "REL $\sim$ FSHP + as.factor $(A A) "$

models $[$ [5] ] = lm(REL AA, training data)

model_names $[5]=$ "REL $\sim A A "$

models $\bar{s}[6]]=\operatorname{lm}(\mathrm{REL} \sim \mathrm{E} 20+$ as.factor(AA), training_data)

model names $[6]=$ "REL $\sim \mathrm{E} 20$ + as.factor $(A A) "$

$\operatorname{model} \bar{s}[[7]]=\operatorname{lm}(\mathrm{REL} \sim \mathrm{E} 6+\operatorname{as}$. factor(AA), training_data)

model names [7] = "REL E6 + as.factor (AA)"

$\operatorname{model} \bar{s}[[8]]=\operatorname{lm}(\mathrm{REL} \sim \mathrm{E} 20+\mathrm{E} 6+$ as.factor(AA), training_data)

model names [8] = "REL $\sim \mathrm{E} 20$ + E6 + as.factor (AA)"

$\operatorname{model} \bar{s}[[9]]=\operatorname{lm}(\operatorname{REL} \sim \mathrm{E} 20+\mathrm{E} 6$, training_data) 


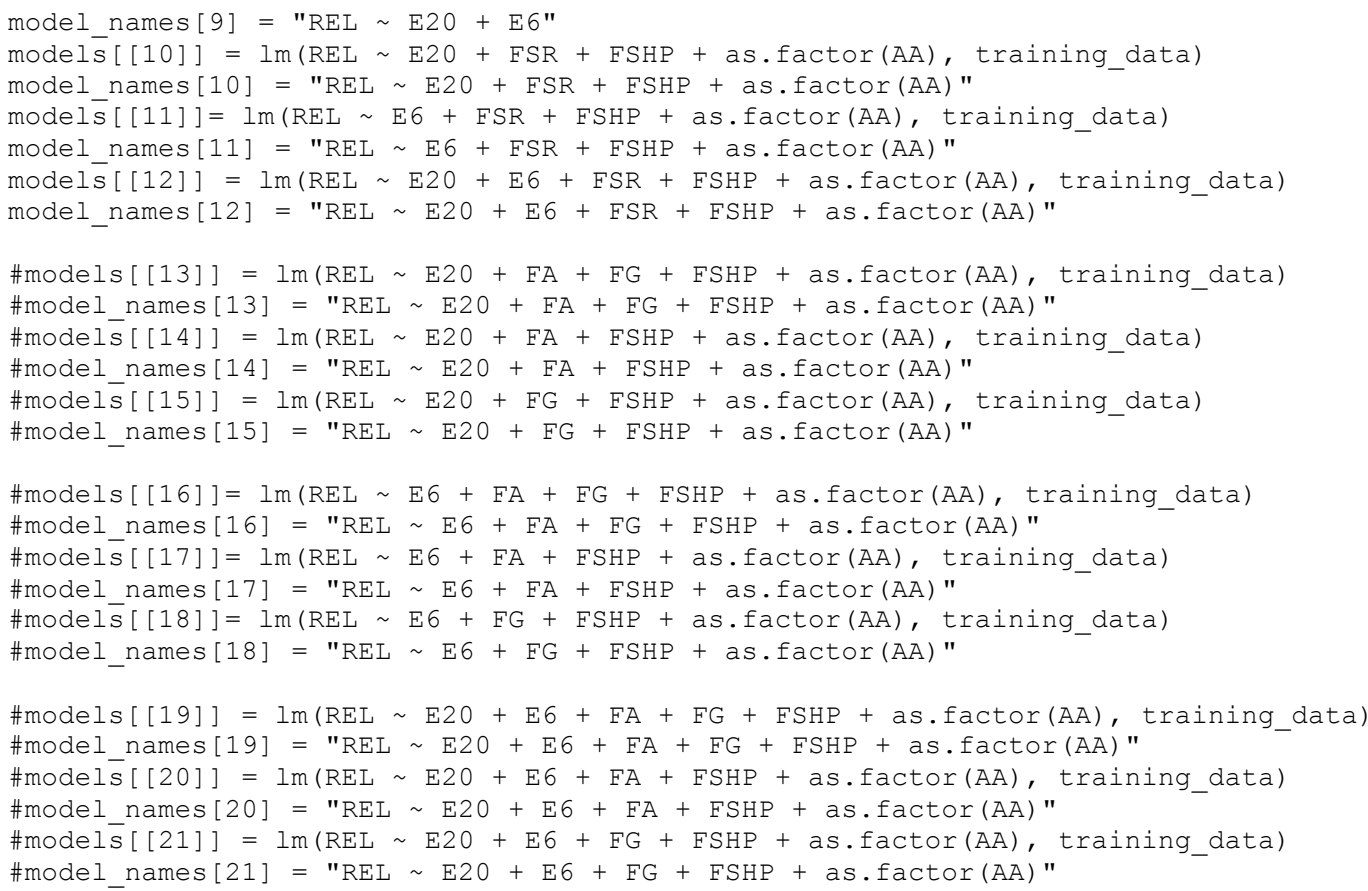




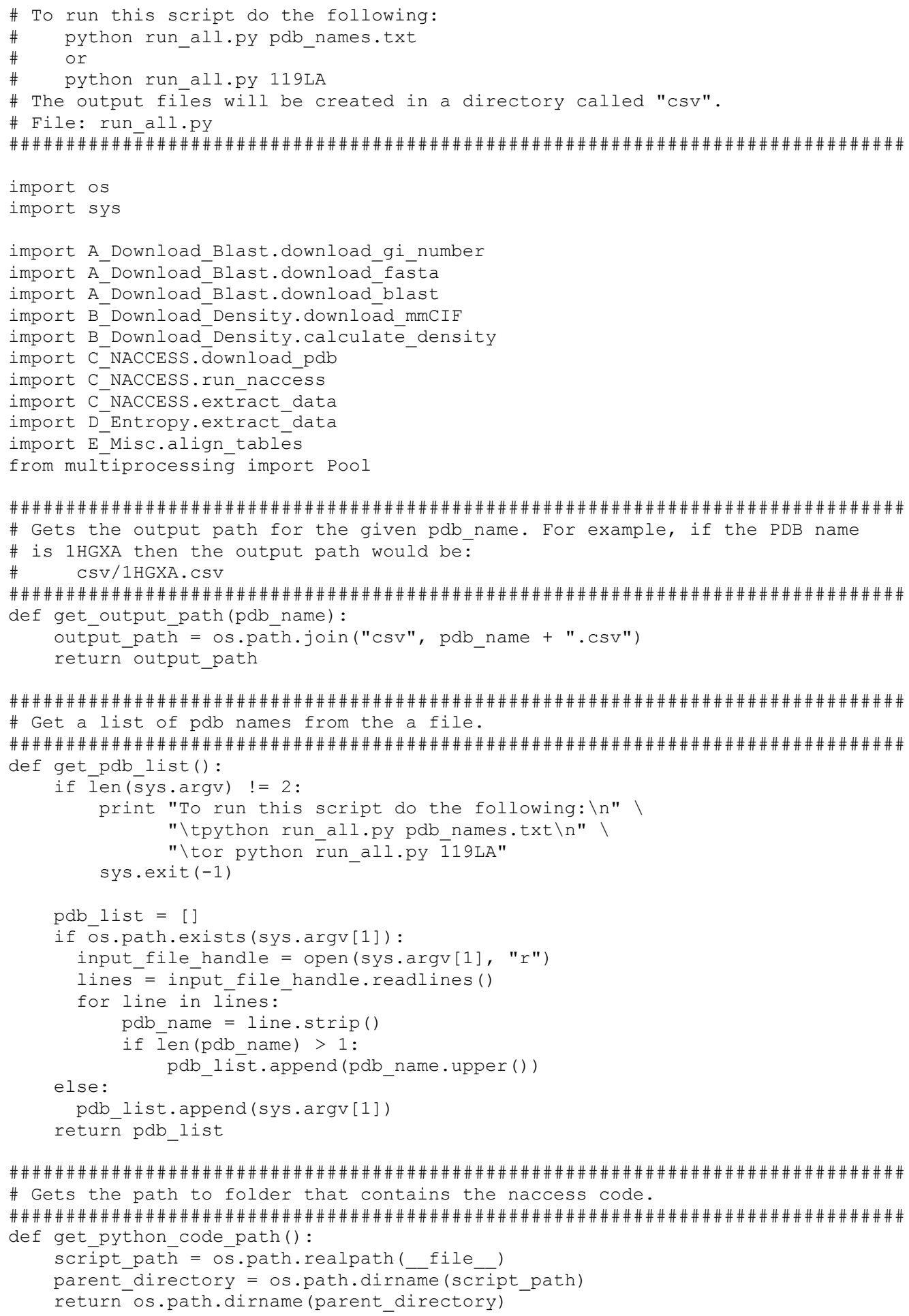

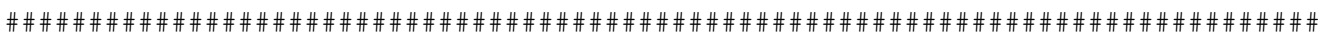
\# Runs the main script.

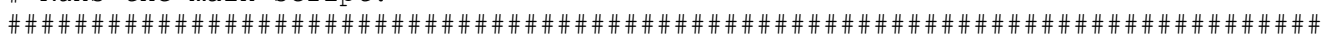
def save_data_to_csv(pdb_name, data, output_path): $f=\overline{o p e n}\left(\overline{o u t} \bar{p} u t \_\right.$path, 'w') 
f.write ('Num, denB, RES, E, E6, FSR, FA, FG, FSHP, EntropyQueryLetter, AA, REL, CATH \n' ) index $=0$

for record in data:

index $=$ index +1

f.write ('od,' $\%$ (index))

f.write ('\%s, ' $\frac{\circ}{\circ}$ record ['denB'])

f.write ('\%s,' \% pdb_name)

f.write ('\%.3f,' \% rēcord ['E'])

f.write ('\%.3f,' \% record ['E6'])

f.write ('\%f,' $\%$ record ['FSR'])

f.write (' $\% f, '$ record ['FA'])

f.write ('\%f,' \% record ['FG'])

f.write ('\%f,' $\%$ record ['FSHP'])

f.write ('os,' \% record ['QueryLetter'])

f.write ('\%s, ' \% record ['AA'])

f.write ('\%s, ' \% record ['REL'])

f.write ('\% ' $\%$ record ['CATH'])

f.write (' $\backslash \mathrm{n}$ ')

f.close ()

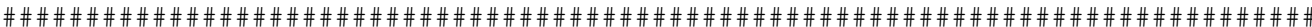

\# Runs the main script.

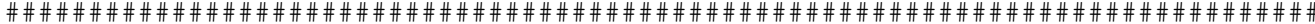

def run(pdb_name):

output path = get output path (pdb name)

if os.path.exists (output_path): return

A Download Blast.download gi number.run (pdb name)

A Download Blast.download_fasta.run (pdb_name)

A Download_Blast.download_blast.run (pdb-name)

B Download Density.downloād mmCIF.run (pōb name)

B_Download_Density.calculate_density.run (pōb_name)

C ${ }^{-}$NACCESS. $\bar{d}$ ownload pdb.run ( $\mathrm{p} \overline{\mathrm{d}} \mathrm{b}$ name)

$\mathrm{C}_{-}{ }^{-} \mathrm{NACCESS} . r u n \_$naccēss.run (pdb_ñame)

(density_table, entropy_table) =

D Entropy.extract dāta. GetEntropyAndDensityValuesForPDB (pdb name)

naccess_table = C_NACCESS.extract_data.GetNaccessValuesForPDB (pdb_name)

aligned_table = E_Misc.align_tablēs.align_tables (density_table,

entropy table, naccess_table)

save_data_to_csv(pdb_name, aligned_table, output_path)

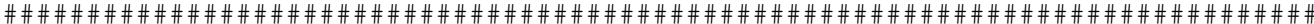

\# The main function, this gets run first when the program is run from the

\# command line.

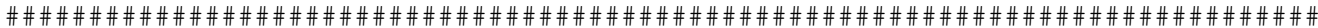

if name = = "main ":

python_code_path = get_python_code_path ()

\# put the file in a directory named "CSV"

if not os.path.exists ("csv"):

os.makedirs ("csv")

pdb list $=$ get $p d b$ list ()

poō $=\operatorname{Pool}($ proces ses $=8$ )

pool.map (run, pdb list)

\#for pab in pdb_list:

\# print pdb

\# $\operatorname{run}(\mathrm{pdb})$

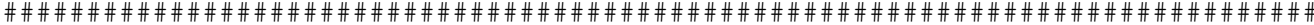

\# Author: Reecha Nepal

\# Date: February 5, 2012

\# Purpose: This program takes entropy data for multiple residues and for each residue 


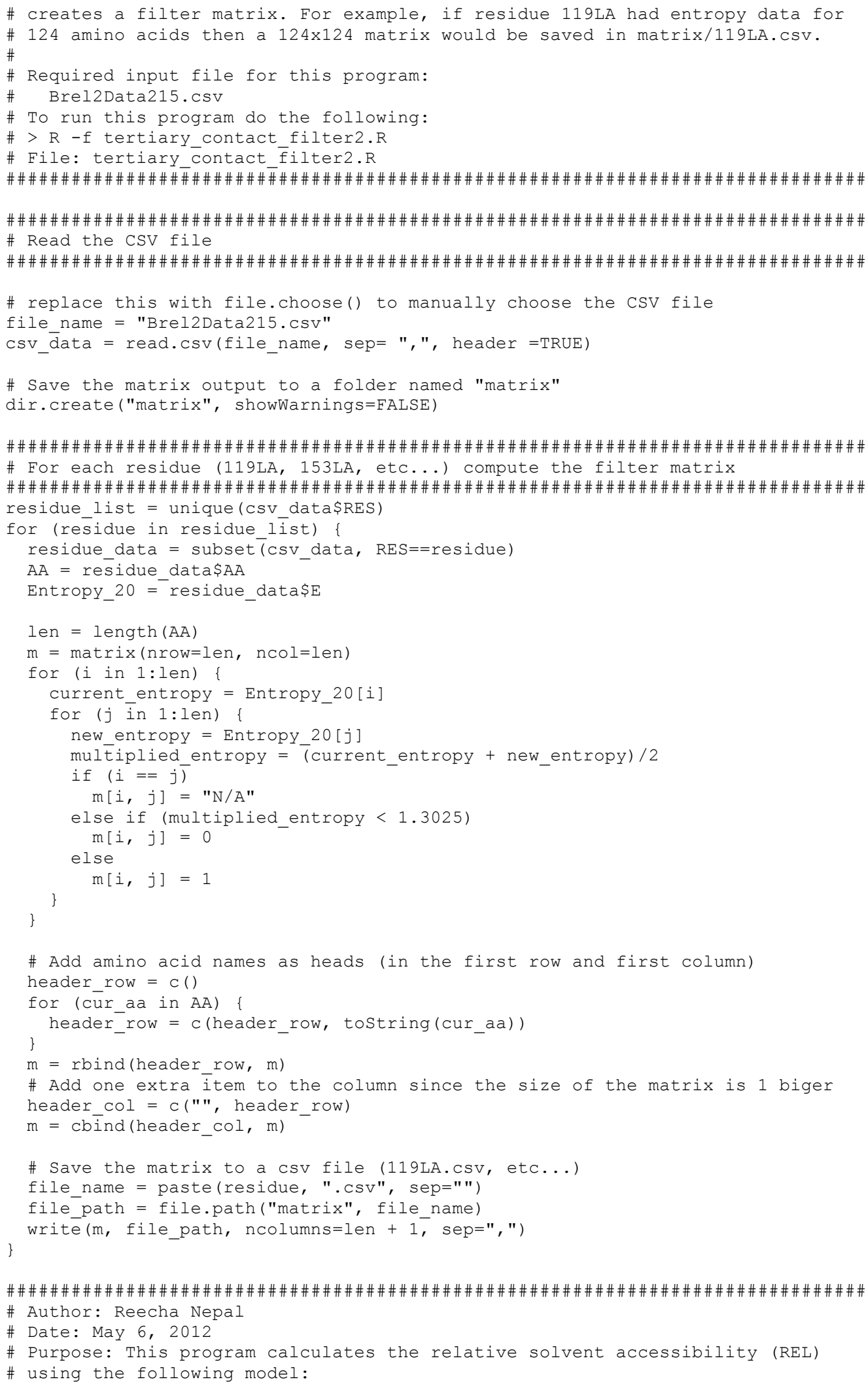




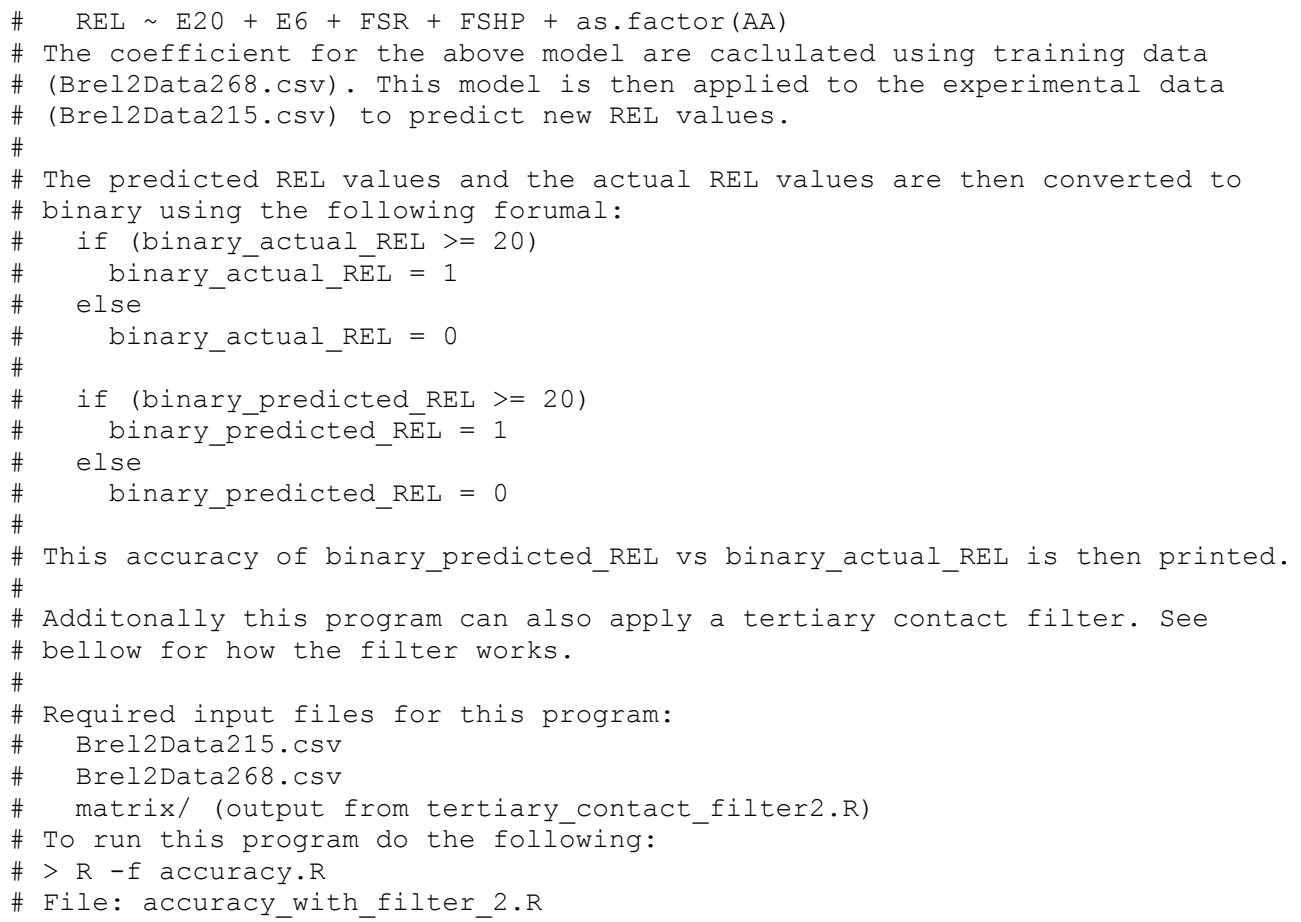

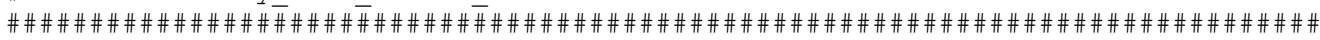

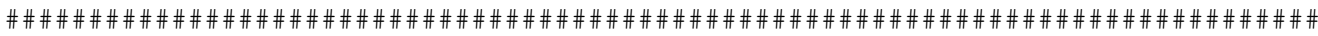
\# Read the training and experimental data.

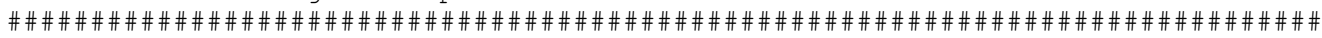
\# replace this with file.choose() to manually choose the CSV file

file_name_268 = "Brel2Data268.csv"

file name $215=$ "Brel2Data215. csv"

blast_268_data = read.csv(file_name_268, sep = ",", header $=$ TRUE)

blast 215 data $=$ read. Csv (file name 215, sep $=", "$, header $=$ TRUE)

blast_268_data_frame $=$ data.frame $(\mathrm{E} 20=$ blast_268_data $\$ \mathrm{E}$,

$\mathrm{E} 6=$ blast_268 data\$E6,

FSR = blast $26 \overline{8}$ data\$FSR,

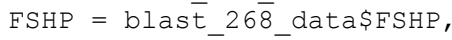

$\mathrm{AA}=$ blast $2 \overline{6} 8$ data\$AA, blast 215 data frame = data.frame $(\mathrm{E} 20=$ blast 215 data\$E,

$\mathrm{E} 6=$ blast_215_- data\$E6,

$\mathrm{FSR}=$ blast $21 \overline{5}$ data\$FSR,

$\mathrm{FSHP}=$ blast $21 \overline{5}$ data\$ESHP,

$\mathrm{AA}=$ blast $2 \overline{1} \overline{5}$ data $\$ \mathrm{AA}$,

REL = blast_21 $\overline{5}$ data\$unREL,

RES = blast_215_data\$RES)

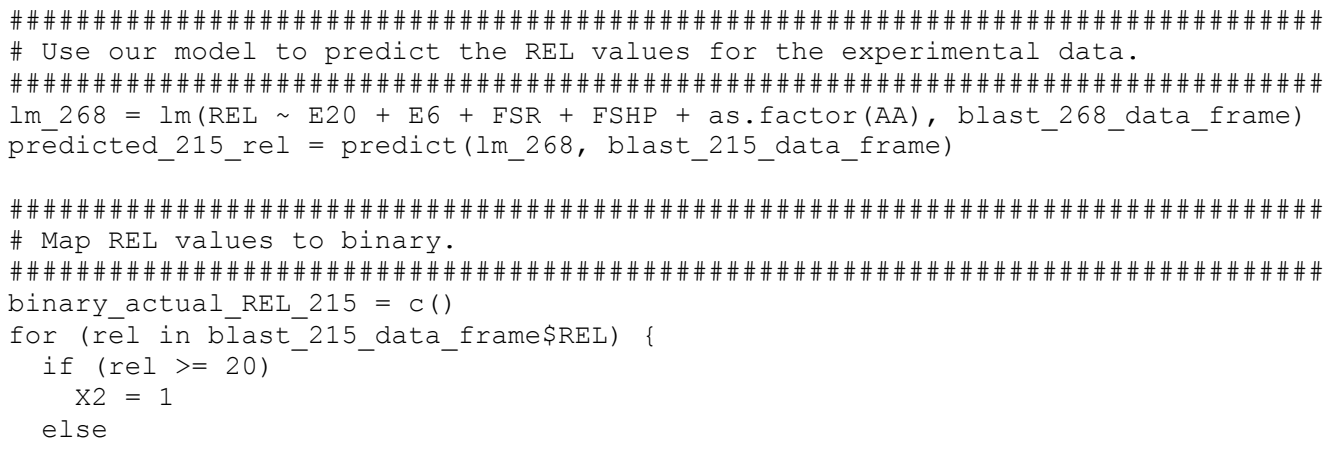




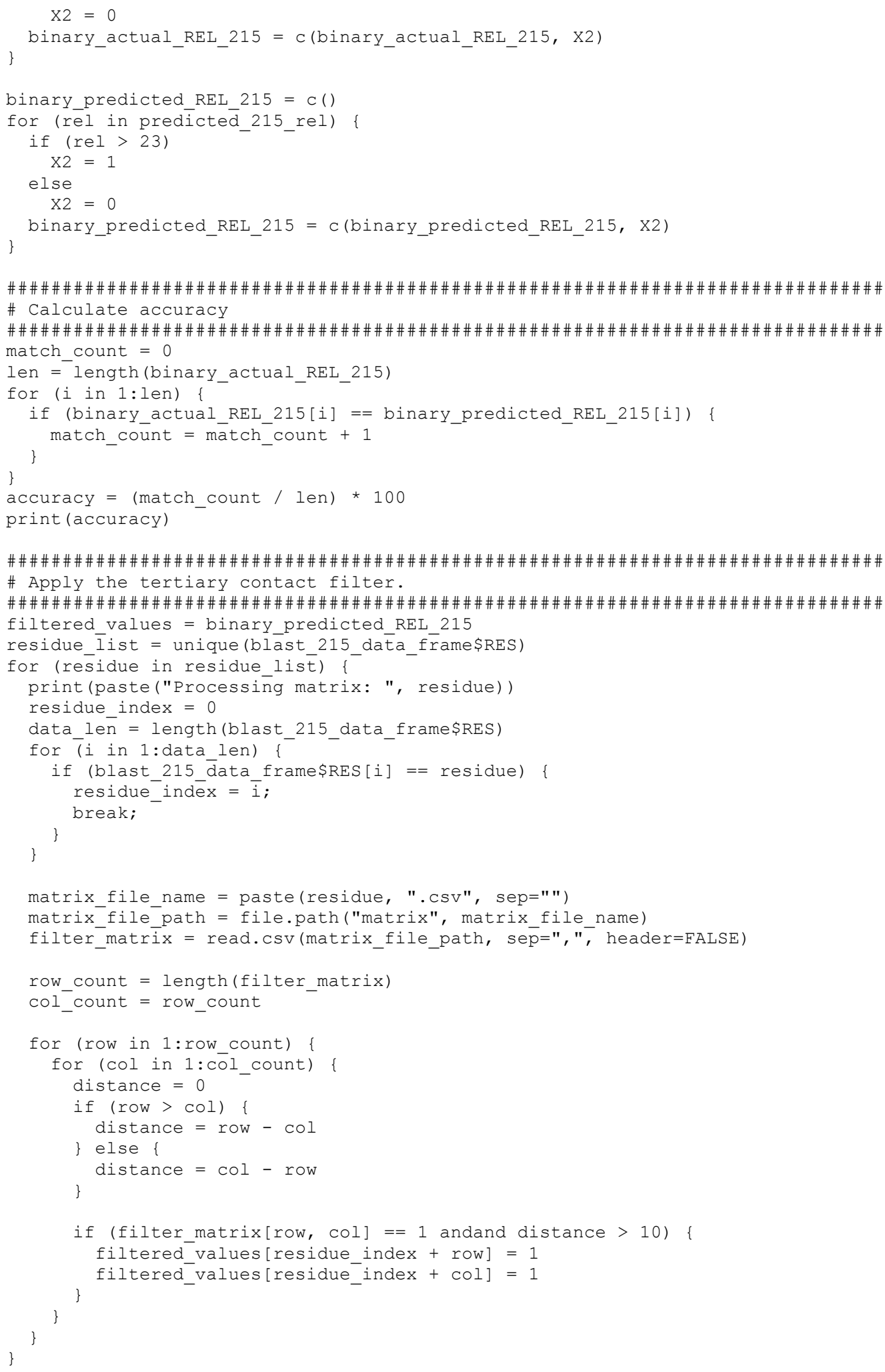

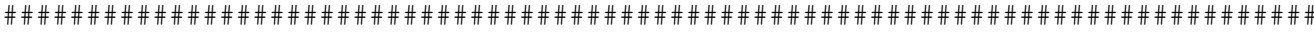




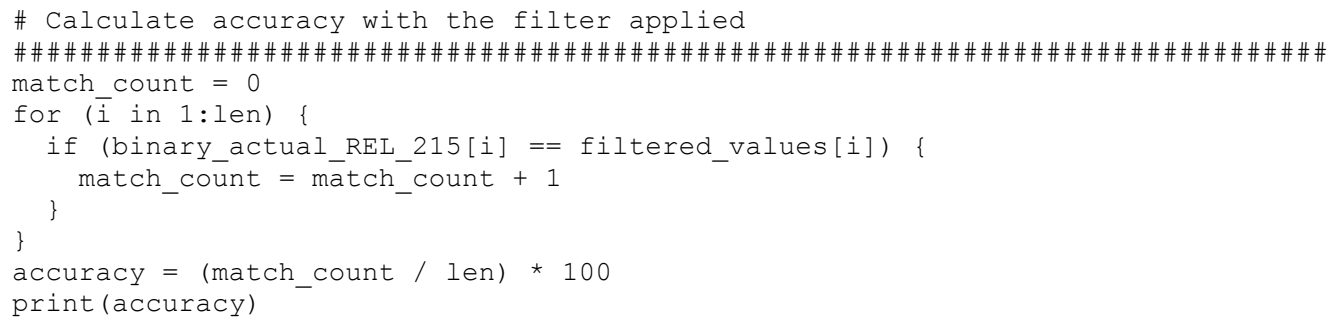

\section{B. 1363 PDB Table}

Table B.1: List of 1363 PDB IDs used in the 1363 training data set.

\begin{tabular}{|c|c|c|c|c|c|c|c|c|}
\hline 2VB1A & $1 \mathrm{KQPA}$ & 1R0RI & $2 \mathrm{R} 2 \mathrm{ZA}$ & 1OQVA & 1V9YA & 2GYQA & $1004 \mathrm{~A}$ & 1UPQA \\
\hline 2DSXA & 1UOWA & 1JM1A & 2BMOA & $1 \mathrm{~F} 4 \mathrm{PA}$ & 1PLCA & 3EJVA & $2 \mathrm{~F} 22 \mathrm{~A}$ & $3 \mathrm{SEBA}$ \\
\hline 1R6JA & $3 \mathrm{C} 70 \mathrm{~A}$ & $1 \mathrm{~K} 7 \mathrm{CA}$ & 1FYEA & $1 \mathrm{G} 61 \mathrm{~A}$ & $1 \mathrm{KKOA}$ & 2P8IA & 1PZ7A & 3C9UA \\
\hline 2B97A & 1PSRA & $1 \mathrm{H} 1 \mathrm{NA}$ & $1 \mathrm{NZOA}$ & $2 \mathrm{C} 4 \mathrm{BA}$ & $1 \mathrm{VH} 5 \mathrm{~A}$ & 1U7IA & 2HQXA & 1WPUA \\
\hline $1 \mathrm{GCIA}$ & 1YQSA & 1SU7A & 1TU9A & 1FCYA & 1UTGA & 2FTRA & 2CJTA & $1 \mathrm{H} 41 \mathrm{~A}$ \\
\hline 2F01A & 3SILA & 2EUTA & 1QU9A & 1XUBA & 1EAJA & $2 \mathrm{~J} 5 \mathrm{YA}$ & 1E6UA & 1UUQA \\
\hline 1G6XA & 1FSGA & 1U07A & 1QW9A & 1K3YA & 2IYVA & 10DZA & $1 \mathrm{JBOA}$ & $10 F 8 \mathrm{~A}$ \\
\hline 1MUWA & 1R2QA & $1 \mathrm{P} 6 \mathrm{OA}$ & $2 \mathrm{~A} 26 \mathrm{~A}$ & 2PRVA & $2 \mathrm{FS} 6 \mathrm{~A}$ & $2 \mathrm{~J} 73 \mathrm{~A}$ & 2GDGA & 1HD2A \\
\hline 1DY5A & 1RQWA & 2UUYA & 2IFQA & $10 \times 0 \mathrm{~A}$ & 1WDDA & 1EZGA & $1 \mathrm{Z} 72 \mathrm{~A}$ & 1OCYA \\
\hline $1 \mathrm{G} 66 \mathrm{~A}$ & 1LQTA & 2V7FA & $1 \mathrm{I} 24 \mathrm{~A}$ & 1WPNA & $1 \mathrm{WDDS}$ & 1M1FA & 1FM0D & 1OFZA \\
\hline 1IX9A & $1 \mathrm{M} 2 \mathrm{DA}$ & $1 \mathrm{I} 8 \mathrm{OA}$ & 1O7IA & 1LQ9A & 2PHNA & 1Q5YA & 1WL8A & 3BI1A \\
\hline 1VYRA & 1EUWA & 1VZIA & 1V8HA & 1NXMA & 1U9CA & 2HIYA & $1 \mathrm{~W} 1 \mathrm{HA}$ & 1ZKPA \\
\hline 2BW4A & 2AXWA & 1I4UA & $1 \mathrm{LC} 0 \mathrm{~A}$ & $1 \mathrm{~J} 2 \mathrm{RA}$ & 2FXUA & $256 \mathrm{BA}$ & $1 \mathrm{KW} 3 \mathrm{~B}$ & $2 \mathrm{O} 02 \mathrm{~A}$ \\
\hline 1OEWA & 1KJQA & 1XODA & 1MJUL & $2 \mathrm{~F} 69 \mathrm{~A}$ & $1 \mathrm{WZDA}$ & 2ACFA & 1Q0RA & 2OPLA \\
\hline 1N9BA & 1PMHX & 1H4AX & $1 \mathrm{~W} 7 \mathrm{CA}$ & 1FLMA & 2PIEA & 2IVYA & $209 \mathrm{CA}$ & 2FNUA \\
\hline 2PVBA & 2C9VA & 2D8DA & $10 I 7 \mathrm{~A}$ & 1R0MA & $1 \mathrm{P} 4 \mathrm{CA}$ & $2 \mathrm{HOXA}$ & 2HEWF & 1LV7A \\
\hline 2PPNA & 1XJUA & $1 \mathrm{M} 1 \mathrm{NB}$ & $1 \mathrm{WMAA}$ & $2 \mathrm{I} 6 \mathrm{CA}$ & 1PZ4A & 2UX9A & 2GLZA & 1KM4A \\
\hline 2FDNA & 1JBEA & 1M1NA & 1USCA & 1SG4A & 1UZKA & 1L6RA & 1ABAA & 1VL7A \\
\hline 1NLSA & 1UWCA & 1HBNA & 1M4LA & 1KMTA & 1PINA & 1YE8A & 1XD3A & $1 \mathrm{~J} 8 \mathrm{UA}$ \\
\hline 2GUDA & $1 \mathrm{~W} 23 \mathrm{~A}$ & 1HBNB & 1RWHA & 2NR7A & 3BUXB & 1K3IA & 1XD3B & $1 \mathrm{P} 3 \mathrm{CA}$ \\
\hline 7А3HA & 1DS1A & $1 \mathrm{HBNC}$ & 1GNLA & 2Q9OA & 1IJYA & $1 \mathrm{KS} 8 \mathrm{~A}$ & 1GVDA & 2PBDP \\
\hline 1RTQA & 1K5NA & 1H4XA & $3 \mathrm{CHBD}$ & 2GF3A & 1KUFA & $1 \mathrm{M} 55 \mathrm{~A}$ & 1OS6A & 1M7JA \\
\hline 1NKIA & 1QLWA & 1X9IA & 2HBAA & 1RYAA & 2AKZA & 1UQ5A & 1JI7A & 1DJ0A \\
\hline 2Z6WA & 1N62B & $1 \mathrm{C} 9 \mathrm{OA}$ & $1 \mathrm{~K} 3 \mathrm{XA}$ & 1JNDA & 1RHSA & 2GMNA & 1DI6A & 2HSJA \\
\hline 1AHOA & $1 \mathrm{~N} 62 \mathrm{C}$ & 1KQ6A & 1EAQA & 2NLVA & 1VIMA & 1Y8AA & 2CVDA & $1 \mathrm{X} 82 \mathrm{~A}$ \\
\hline $1 \mathrm{XG0C}$ & $1 \mathrm{~N} 62 \mathrm{~A}$ & 1UWKA & 1OOHA & 3BBBA & $1 \mathrm{JO} 0 \mathrm{~A}$ & 2QFAA & 1HZTA & 2BT6A \\
\hline 1XG0A & 1ZL0A & $2 \mathrm{CS} 7 \mathrm{~A}$ & 1MF7A & 108XA & 1F1EA & 1EW4A & $1 \mathrm{~W} 5 \mathrm{RA}$ & $2 \mathrm{~J} 2 \mathrm{JA}$ \\
\hline 2NMZA & 1ITXA & 1WRIA & $2 \mathrm{C} 60 \mathrm{~A}$ & $3 \mathrm{D} 32 \mathrm{~A}$ & 3CJSB & 1UWWA & 1THFD & $2 \mathrm{~F} 62 \mathrm{~A}$ \\
\hline 1IXHA & $1 \mathrm{I} 40 \mathrm{~A}$ & $1 \mathrm{MJ} 4 \mathrm{~A}$ & $2 \mathrm{~B} 82 \mathrm{~A}$ & 1HYOA & 3CJSA & 1JUBA & 1IDPA & 109GA \\
\hline
\end{tabular}




\begin{tabular}{|c|c|c|c|c|c|c|c|c|}
\hline 1MWQA & 2V9VA & 1VR7A & $2 \mathrm{FVVA}$ & 1ES9A & 1GVEA & 1T7RA & $1 \mathrm{IO} 0 \mathrm{~A}$ & 2IMJA \\
\hline 1P1XA & $2 \mathrm{~V} 89 \mathrm{~A}$ & 1WM3A & 2QJZA & 2FCJA & 1RYQA & $2 \mathrm{C} 78 \mathrm{~A}$ & 1V4PA & 1KQ3A \\
\hline 1JFBA & 2BHUA & 1XDNA & 2TPSA & 1I0DA & 1JKVA & 1PFBA & 1URSA & 1ISUA \\
\hline $107 \mathrm{JA}$ & 1G8TA & $1 \mathrm{KG} 2 \mathrm{~A}$ & 1C1DA & 1HDHA & 2BFDA & 2TNFA & 2RL8A & 2BRJA \\
\hline 1GA6A & 1SBYA & 2BLNA & 2G3RA & 1UCDA & 2BFDB & 1NYCA & 1VE1A & $2 \mathrm{~J} 8 \mathrm{KA}$ \\
\hline 1Q6ZA & 1Z2UA & 1UCRA & 2GOMA & $1 \mathrm{~V} 70 \mathrm{~A}$ & 1N8VA & 2OHWA & 1VMGA & $2 \mathrm{Z} 3 \mathrm{GA}$ \\
\hline 2RBKA & 2ABSA & 1Q6OA & 108BA & 2GECA & $1 \mathrm{I} 1 \mathrm{JA}$ & 1PWBA & 1HNJA & 1XRKA \\
\hline 2JHFA & 1LS1A & 2VPAA & 1QAUA & 1VDWA & 1SJYA & $1 \mathrm{GP} 0 \mathrm{~A}$ & 1QREA & 1CRUA \\
\hline 2IIMA & 1CTJA & 1NWWA & 1RDQE & $2 \mathrm{PV} 2 \mathrm{~A}$ & 1WB4A & 1NG6A & 1G3PA & $2 \mathrm{~A} 35 \mathrm{~A}$ \\
\hline $1 \mathrm{NQJA}$ & 1BKRA & $2 \mathrm{IJ} 2 \mathrm{~A}$ & 1GXUA & 1VD6A & $2 \mathrm{G} 84 \mathrm{~A}$ & 1ES5A & 2R8OA & 2PYQA \\
\hline 1OAIA & 1T2DA & $1 \mathrm{~W} 6 \mathrm{SA}$ & $2 \mathrm{~J} 8 \mathrm{WA}$ & 1JL1A & 2ODKA & $1 \mathrm{FP} 2 \mathrm{~A}$ & $1 \mathrm{JU} 2 \mathrm{~A}$ & 2IBAA \\
\hline $1 \mathrm{C} 7 \mathrm{KA}$ & 1UZ3A & $1 \mathrm{~W} 6 \mathrm{SB}$ & 1GKPA & 1GXMA & $2 \mathrm{G} 7 \mathrm{SA}$ & 2POFA & $104 \mathrm{YA}$ & $2 \mathrm{FOMB}$ \\
\hline 1CXQA & 1RG8A & 2DLBA & 2AEBA & 1E7LA & 1K0MA & 1XSZA & 1VM9A & 3ER7A \\
\hline 1JR8A & 1F0LA & $2 \mathrm{~F} 1 \mathrm{KA}$ & 1HFES & $1 \mathrm{WQJB}$ & 2JE6I & 2AALA & $1 \mathrm{VJOA}$ & 1PFVA \\
\hline 1IRQA & 1SMBA & 2F6UA & 2EZ9A & 1WQJI & 1Y1PA & 2D29A & 2JE8A & $1 \mathrm{~V} 58 \mathrm{~A}$ \\
\hline 1CIPA & 2ZKDA & $2 \mathrm{HX} 0 \mathrm{~A}$ & 1MXGA & $1 \mathrm{~V} 2 \mathrm{BA}$ & 1RYLA & 1VKFA & 2A9DA & $1 \mathrm{WO} A \mathrm{~A}$ \\
\hline 1NTVA & 1PTFA & $2 \mathrm{~V} 33 \mathrm{~A}$ & 3ETJA & 1G8QA & 1JKXA & 2QEEA & 2GDMA & $1 \mathrm{~B} 2 \mathrm{PA}$ \\
\hline $1 \mathrm{SX} 5 \mathrm{~A}$ & 1Y7BA & 2NMLA & $2 \mathrm{GUIA}$ & 1B5EA & $1158 \mathrm{~A}$ & $1 \mathrm{H} \times 6 \mathrm{~A}$ & 2OD4A & 3ВYPA \\
\hline $1 \mathrm{JX} 6 \mathrm{~A}$ & 2FHPA & 1MG7A & $1 \mathrm{~K} 92 \mathrm{~A}$ & $1 I U 8 A$ & $1 \mathrm{~N} 08 \mathrm{~A}$ & $2 \mathrm{EXOA}$ & 2NXFA & 1YDYA \\
\hline 1JYKA & 2D5JA & $1 I X 2 A$ & $2 \mathrm{PY} 5 \mathrm{~A}$ & 1LQVA & 1XPPA & 1Y7TA & 1EEXA & 1XSVA \\
\hline 1FL0A & 1RKIA & 1JKEA & 1U8VA & 1J1NA & 1KAFA & $10 K 7 \mathrm{~A}$ & $1 \mathrm{EEXB}$ & 1RXQA \\
\hline 1Y5HA & $2 \mathrm{FL} 4 \mathrm{~A}$ & $2 \mathrm{HHVA}$ & 1S1DA & 1QGIA & 1YD9A & 2EVOA & 1EEXG & 1BUPA \\
\hline 1GMUA & 1WOPA & $1 \mathrm{~A} 62 \mathrm{~A}$ & 2VLQB & $1 \mathrm{WS} 8 \mathrm{~A}$ & $1 \mathrm{M} 4 \mathrm{JA}$ & 2DEKA & 2ONFA & $1 \mathrm{NVMB}$ \\
\hline 2INWA & 2G5RA & 1UGIA & 2VLQA & 1YXYA & $1 \cup 60 \mathrm{~A}$ & 1UEBA & 2RLDA & $1 \mathrm{DC} 1 \mathrm{~A}$ \\
\hline 1V2XA & 3DMCA & 10QJA & 1D3GA & 1T9IA & 1XKGA & 1VHQA & 2ID3A & 1XCRA \\
\hline 1QS1A & 2FHFA & $1 \mathrm{BI} 5 \mathrm{~A}$ & 1TJOA & $3 \mathrm{C} 8 \mathrm{WA}$ & 2QFKA & 1GXRA & $1 \mathrm{VCLA}$ & 1OGQA \\
\hline 1NLQA & 2FMPA & $2 \mathrm{~V} 3 \mathrm{ZA}$ & 1P0ZA & 1QMGA & $2 Z K M X$ & $1 \mathrm{VIOA}$ & 1JHDA & $2 \mathrm{FA} 1 \mathrm{~A}$ \\
\hline 1OMRA & 1FDRA & 1VKEA & 2ITEA & 1YT3A & $2 \mathrm{CJ} 4 \mathrm{~A}$ & 1UFIA & 2R85A & 1QD1A \\
\hline $1 \mathrm{C} 4 \mathrm{OA}$ & 10Q1A & 1SXRA & $2 \mathrm{BZ6L}$ & 1E2WA & 1E5PA & $1 \mathrm{FXOA}$ & 1YOCA & $1 \mathrm{H} 03 \mathrm{P}$ \\
\hline 3BEXA & 1DMHA & 2AFWA & $1 \mathrm{~F} 74 \mathrm{~A}$ & $21 \cup 5 A$ & 2ICTA & 1VPMA & $1 \mathrm{UDDA}$ & $1 \mathrm{G} 3 \mathrm{MA}$ \\
\hline 2OOCA & 1S5UA & 2BKFA & $1 T 92 \mathrm{~A}$ & 1PE9A & 2FBNA & $1 \mathrm{GZGA}$ & 1WDVA & $1 \mathrm{HZ6A}$ \\
\hline 1C4QA & 1T1JA & $2 \mathrm{~F} 9 \mathrm{HA}$ & 1T4BA & 2BDRA & 1PU6A & 1L3LA & 1P3DA & 1RH6A \\
\hline 2FSRA & 1NKRA & 1JOVA & 1X7DA & $1 \mathrm{~J} 2 \mathrm{JA}$ & $1 \mathrm{G} 8 \mathrm{KA}$ & $2 \mathrm{AJ} 7 \mathrm{~A}$ & 1D1QA & 1DUVG \\
\hline 2JFGA & $1 \mathrm{NSZA}$ & 2GUYA & $1 \mathrm{~S} 95 \mathrm{~A}$ & $1 \mathrm{WLZA}$ & 3ELGA & 1N1JA & 2IDLA & 2DUCA \\
\hline 1QQ5A & $2062 A$ & $2 \mathrm{IA} 1 \mathrm{~A}$ & $2 N W 8 A$ & 1ZKEA & 1YLLA & $2 \mathrm{R} 7 \mathrm{GA}$ & 2BKYA & 2NUGA \\
\hline $1 \mathrm{~W} 7 \mathrm{BA}$ & $216 \mathrm{HA}$ & $1 \mathrm{VIOA}$ & 1FIUA & $1 \mathrm{~J} 2 \mathrm{JB}$ & 1VPDA & 1UD9A & $1 \mathrm{GU7A}$ & $1 \mathrm{~T} 82 \mathrm{~A}$ \\
\hline 2R1JL & 2J9UA & $1 \mathrm{~K} 7 \mathrm{IA}$ & 1TQJA & $1 \mathrm{GY} 7 \mathrm{~A}$ & 1DWKA & 1TH7A & 2AVDA & $1 \mathrm{GQA}$ \\
\hline 1L2HA & 2J9UB & 2NNUA & 1YOUA & 1RFYA & $2 \mathrm{G} 50 \mathrm{~A}$ & 1JP4A & 1P1JA & 3BOFA \\
\hline 1VHWA & $1 \mathrm{GXJA}$ & $1 \mathrm{CCWB}$ & 1TOAA & 1T1VA & $1 \mathrm{~W} 2 \mathrm{YA}$ & $218 D A$ & 10OYA & 1UV7A \\
\hline
\end{tabular}




\begin{tabular}{|c|c|c|c|c|c|c|c|c|}
\hline 2B5AA & 1WMXA & 1CCWA & $1 \times 60 A$ & 2CPGA & $1 \mathrm{IM} 5 \mathrm{~A}$ & $1 J Y 1 A$ & $2 \mathrm{EG} 6 \mathrm{~A}$ & 1SGWA \\
\hline 2GHSA & $1 \mathrm{G} 73 \mathrm{~A}$ & 2OKFA & $10 W 4 A$ & $1026 \mathrm{~A}$ & $2 \mathrm{G} 82 \mathrm{~A}$ & 2HFTA & 1JW9B & 1VFJA \\
\hline 1SFXA & $1 \mathrm{JC} 4 \mathrm{~A}$ & 1LQAA & 1KQFA & 1M7YA & $1054 \mathrm{~A}$ & 2QIYC & $2 I G I A$ & 1DQGA \\
\hline $2 \mathrm{~F} 71 \mathrm{~A}$ & 1DQTA & 1T9HA & 2POSA & $2 \mathrm{~F} 23 \mathrm{~A}$ & $2 \mathrm{P} 97 \mathrm{~A}$ & 3BYQA & 1QXMA & 1P99A \\
\hline 2BEMA & 1XOPA & 2APJA & 1JNRA & 1WC9A & $3 C L S D$ & 1TOBA & 2DVTA & 1V9FA \\
\hline 1WBHA & $2 \mathrm{PI} 2 \mathrm{~A}$ & $210 \mathrm{KA}$ & 1ELWA & $2 \mathrm{~V} 5 \mathrm{ZA}$ & 3 CLSC & 1YKIA & $1 \mathrm{D02A}$ & 1KZQA \\
\hline 1W6GA & 1TJLA & $2 \mathrm{BO} 9 \mathrm{~B}$ & 1L9XA & $1 \mathrm{JHJA}$ & 1QSAA & $2151 \mathrm{~A}$ & 2VAPA & 1XKPA \\
\hline $1 \mathrm{MV} 8 \mathrm{~A}$ & $2 \mathrm{PI} 2 \mathrm{E}$ & $2 \mathrm{CL} 5 \mathrm{~A}$ & $2 \mathrm{~J} 43 \mathrm{~A}$ & $20 Y 9 A$ & $2 I \mathrm{MLA}$ & $2 \mathrm{CCHB}$ & 1MTYD & 1P5VA \\
\hline 2FP1A & $1 \mathrm{H} 3 \mathrm{FA}$ & $1 \mathrm{VKIA}$ & 1UKKA & $10 \cup 8 \mathrm{~A}$ & $1 \mathrm{KOLA}$ & 1TXGA & 1MTYB & $20 \times 6 \mathrm{~A}$ \\
\hline 1EJDA & 1YOZA & $20 \mathrm{MZA}$ & 1AOPA & $1 \mathrm{IT} 2 \mathrm{~A}$ & 1NNHA & $2 \mathrm{~B} 8 \mathrm{MA}$ & $2 \mathrm{CB} 2 \mathrm{~A}$ & 1ZMTA \\
\hline 2ELCA & 1F2LA & 2OMZB & $1 \mathrm{~V} 7 \mathrm{ZA}$ & 2B9DA & 1S99A & 108FA & 1MTYG & $1 \mathrm{~K} 94 \mathrm{~A}$ \\
\hline 1VLPA & $20 \times 7 \mathrm{~A}$ & 2NP5A & $2 \mathrm{FBHA}$ & $2 \mathrm{~A} 1 \mathrm{HA}$ & $1033 \mathrm{~A}$ & 1T15A & 2JDIA & $1 \mathrm{CB} 8 \mathrm{~A}$ \\
\hline $2174 \mathrm{~A}$ & 2GIYA & 1TDZA & $10 B 8 A$ & $1 \mathrm{~A} 73 \mathrm{~A}$ & $2 Q J 2 A$ & 3ENBA & 2JDID & 1UHGA \\
\hline 2F1FA & $2 \mathrm{BS} 2 \mathrm{~A}$ & 1YOBA & 109UA & $114 \mathrm{JA}$ & 1GQEA & 1U7KA & 1Q0QA & $1 \mathrm{~T} 06 \mathrm{~A}$ \\
\hline 1KPTA & $2 \mathrm{BS} 2 \mathrm{C}$ & 1BEBA & 3ERJA & 1PVGA & $2 \mathrm{JI7A}$ & 2B3YA & $1 \mathrm{CHMA}$ & 2PF5A \\
\hline 1JAKA & 2BS2B & 2IT9A & 1ORUA & 1V4VA & $1 \mathrm{~B} 65 \mathrm{~A}$ & $1 C Q 3 A$ & 2JDIG & 1FL2A \\
\hline 2FPWA & $1 \times 74 \mathrm{~A}$ & $1 \mathrm{~N} 7 \mathrm{HA}$ & 2VO9A & 100PA & 1RY9A & 1VCTA & $2 \mathrm{JDIH}$ & $1 \mathrm{M} 6 \mathrm{YA}$ \\
\hline $1 \mathrm{CV} 8 \mathrm{~A}$ & 2NTOA & 1CMCA & $2070 A$ & $1 \mathrm{KXOA}$ & 1WURA & $2 \mathrm{H} 62 \mathrm{~A}$ & 1QB5D & 1YQGA \\
\hline 203FA & 2PBLA & 1FNLA & 1QWRA & 2B8UA & $1 \mathrm{H} 8 \mathrm{PA}$ & $2 \mathrm{CWZA}$ & 2JDII & $1 \mathrm{AYOA}$ \\
\hline 1PG4A & $105 \mathrm{KA}$ & 1WEHA & 2PNXA & $1 U U Z A$ & 1VLRA & $1 \mathrm{~J} 75 \mathrm{~A}$ & 1UYPA & 1OBBA \\
\hline 1PM4A & $1 \mathrm{VK} 8 \mathrm{~A}$ & $2 \mathrm{C} 2 \mathrm{IA}$ & 2GDQA & $1 \mathrm{~K} 6 \mathrm{KA}$ & 1H7EA & 2QMMA & 2ZQNA & 1LR5A \\
\hline 1ZARA & 2NACA & $1 \mathrm{VC} 4 \mathrm{~A}$ & $1 \mathrm{M} 6 \mathrm{SA}$ & $11 \mathrm{Q} 4 \mathrm{~A}$ & $105 U A$ & 1EYQA & 1UNNC & 2PEQA \\
\hline $1 \mathrm{~K} 75 \mathrm{~A}$ & 1VMEA & 1JIDA & 1F1MA & 1FPOA & $1 \mathrm{TX} 2 \mathrm{~A}$ & 2CXKA & 1REGX & $1 \mathrm{G} 3 \mathrm{KA}$ \\
\hline 1UUJA & $2 \mathrm{ACOA}$ & 200KA & $1 \mathrm{~V} 54 \mathrm{~A}$ & $1 I G 0 A$ & $3 \mathrm{E} 5 \mathrm{UA}$ & 2PD1A & 1VQ3A & $2 \mathrm{FA} 8 \mathrm{~A}$ \\
\hline $1 \mathrm{NBCA}$ & 1M3UA & 1IIBA & 1UXYA & 1FS1B & $1069 \mathrm{~A}$ & 1ZPDA & 1YARO & 10TFA \\
\hline 1GTFA & 1NARA & $1 C 3 D A$ & $1 \mathrm{~V} 54 \mathrm{C}$ & $1 \mathrm{G} 8 \mathrm{EA}$ & 2DYJA & 1JB7A & 1YARA & 1VBKA \\
\hline 1NE7A & 1XFFA & 1SW5A & $1 \mathrm{~V} 54 \mathrm{~B}$ & $1 \mathrm{KU} 3 \mathrm{~A}$ & 1JR2A & $1 T Z Z A$ & 219AA & 1J9JA \\
\hline 1YRBA & $2 \mathrm{C} \times 1 \mathrm{~A}$ & 1NZYA & 1VCAA & 1FS1A & 1SS4A & $2 \mathrm{FZPA}$ & 1XEDA & 1AGQA \\
\hline 1TVXA & 1LBUA & $1 \mathrm{VYBA}$ & $1 \mathrm{~V} 54 \mathrm{D}$ & 1NKPA & 1SGJA & $2 \mathrm{~A} 2 \mathrm{MA}$ & $1008 \mathrm{~A}$ & 1BXYA \\
\hline $2 \mathrm{EX} 4 \mathrm{~A}$ & 2RGQA & 2FURA & $1 \mathrm{~V} 54 \mathrm{E}$ & 2FMMA & $1 \mathrm{VKHA}$ & 2PQRA & 2FREA & 3СРТА \\
\hline 2NPTA & 1TROA & $2 A G 4 A$ & $1 \mathrm{~V} 54 \mathrm{~F}$ & 2ZD1B & 20TMA & 2PQRC & 3ECFA & ЗСРТВ \\
\hline 2FUEA & 1ZLQA & 1BDOA & 1V54G & 2NS9A & 1WVEA & 2IGTA & 2NR5A & 2NYCA \\
\hline 1DQNA & 1LSTA & $1 \mathrm{~A} 3 \mathrm{AA}$ & $1 \mathrm{~V} 54 \mathrm{H}$ & 2CYYA & $20 \cup 3 \mathrm{~A}$ & 2GIAA & 1YT8A & $1 \mathrm{U} 6 \mathrm{ZA}$ \\
\hline $1 \mathrm{VH} 4 \mathrm{~A}$ & $2 Q 03 \mathrm{~A}$ & $219 \times A$ & 1V54I & 2R7DA & $1 \mathrm{~B} 25 \mathrm{~A}$ & 1V3EA & 1LUAA & 1FOXA \\
\hline 1WWZA & 1FN9A & 1A9XA & $1 \mathrm{~V} 54 \mathrm{~J}$ & 1RWZA & $2 \mathrm{VZSA}$ & $1 \mathrm{M} 2 \mathrm{~TB}$ & $1091 \mathrm{~A}$ & 1STMA \\
\hline 1NRZA & $1 Y X 1 A$ & 10WLA & $1 \mathrm{~V} 54 \mathrm{~K}$ & 1WZ3A & 2CWYA & $1 \mathrm{VKCA}$ & 2DSYA & $1 \mathrm{MODA}$ \\
\hline $1 \mathrm{GO} 3 \mathrm{~F}$ & 1RQPA & $1 \mathrm{~A} 9 \times \mathrm{B}$ & $1 \mathrm{~V} 54 \mathrm{~L}$ & $2 \mathrm{Z1CA}$ & $2 \mathrm{CHOA}$ & 1JFRA & $2 \mathrm{~A} 6 \mathrm{CA}$ & $1 \mathrm{Cl} 4 \mathrm{~A}$ \\
\hline 1XE7A & 2H1TA & 1DUSA & $1 \mathrm{~V} 54 \mathrm{M}$ & $1 \mathrm{WZ8A}$ & 2 CB5A & $10 A O C$ & $1 \cup 94 \mathrm{~A}$ & 3BDUA \\
\hline 1JLVA & 1MGTA & 1JH6A & 1WDYA & 1L8BA & 1K8WA & 10JXA & 2FEFA & 1G291 \\
\hline
\end{tabular}




\begin{tabular}{|c|c|c|c|c|c|c|c|c|}
\hline 1XKRA & 1YTLA & 1YKSA & $1 \mathrm{LM} 5 \mathrm{~A}$ & $1 \mathrm{CS} 6 \mathrm{~A}$ & 1TUHA & $1 Y Q 2 A$ & 1QMYA & 2GAUA \\
\hline 1L1LA & 1VLAA & 1H4RA & 1UDZA & 1Y9IA & 2IEAA & 1IOWA & 1U7PA & 1Y7PA \\
\hline 2P3PA & 1GUQA & 1WLGA & 1LB6A & $1 \mathrm{~V} 2 \mathrm{ZA}$ & $1 \mathrm{EX} 2 \mathrm{~A}$ & 2MNRA & $1 T Z Y C$ & 1AMUA \\
\hline 3BWUF & 1ATZA & 1RWIA & 2SPCA & 1RKXA & $2 \mathrm{H} 7 \mathrm{XA}$ & $1 \mathrm{JOHA}$ & 1TZYA & $1 \mathrm{~B} 63 \mathrm{~A}$ \\
\hline 3BWUD & 209AA & 1WOQA & $1 \mathrm{NU} 4 \mathrm{~A}$ & 1SQDA & 1LWDA & 1ZQ9A & $1 T Z Y B$ & 1XIWA \\
\hline 2GMQA & $2 \mathrm{HQYA}$ & $1 \mathrm{UJ} 2 \mathrm{~A}$ & $1 \mathrm{~W} 2 \mathrm{FA}$ & $1 \mathrm{LUZA}$ & 20D6A & $1 S Z O A$ & 1TZYD & 1F00I \\
\hline 1YNBA & 1MR7A & 1XTTA & $1 \mathrm{KOIA}$ & $1 \mathrm{H} 8 \mathrm{UA}$ & $1 \mathrm{~V} 82 \mathrm{~A}$ & 2CWQA & 2ODFA & 1IWMA \\
\hline 1U9KA & $2102 \mathrm{~A}$ & 1QNAA & $1 \mathrm{~N} 97 \mathrm{~A}$ & $1 \times 8 D A$ & 1SSQA & $2 \mathrm{CH} 5 \mathrm{~A}$ & 1S4KA & $2 \mathrm{FF} 4 \mathrm{~A}$ \\
\hline 1R7AA & 3BB9A & $1 F X L A$ & $2 \mathrm{H} 26 \mathrm{~A}$ & 1VQQA & 2AUWA & 1VOEA & 1ZPVA & 2ZGYA \\
\hline 3BSWA & $1 \mathrm{C} 3 \mathrm{CA}$ & 1MPGA & 3PROA & $1 \mathrm{VHSA}$ & 1EXTA & 10N3A & 1A8LA & 1NNWA \\
\hline $1 \mathrm{U} 55 \mathrm{~A}$ & 1RYIA & $1 \mathrm{~W} 96 \mathrm{~A}$ & 3PROC & 2GAXA & $2 Z 3 Q A$ & 1PL3A & 2NZWA & 1JYSA \\
\hline 1X7VA & 1VKNA & 1TENA & 2G5FA & $2 \mathrm{GU} 2 \mathrm{~A}$ & $2 \mathrm{Z3QB}$ & 20KUA & 1CEOA & 1DZFA \\
\hline $1 Z 60 A$ & 1TXOA & $1 W 85 I$ & 1HRUA & 1WIWA & $1 \mathrm{VCHA}$ & 2BPTA & 1DEKA & $1 \mathrm{MZGA}$ \\
\hline $1 \mathrm{Z6OM}$ & 1KHYA & 1PPRM & $1136 \mathrm{~A}$ & 1ROWA & 20GQA & $2 S Q C A$ & $1 \mathrm{~N} 2 \mathrm{ZA}$ & 1U5UA \\
\hline 2GF6A & 1JGTA & 1SACA & 1UANA & $1 T Y Z A$ & 1XHNA & 2IHTA & 1WDJA & 1EL6A \\
\hline 2RDEA & 1YNHA & 1Z2WA & $1 \mathrm{~W} 07 \mathrm{~A}$ & 1Q2YA & $2 \mathrm{BO} 4 \mathrm{~A}$ & 2NUJA & $1 \mathrm{KXGA}$ & 1SD4A \\
\hline 1ZELA & 1T6SA & 1RFXA & $3 \mathrm{C} 10 \mathrm{~A}$ & $1 U C 8 \mathrm{~A}$ & $117 Q A$ & 2EBNA & IITUA & $1 \mathrm{KNQA}$ \\
\hline $1 \mathrm{WC1A}$ & 2АРОB & $2 S C P A$ & $2 \mathrm{BB} 0 \mathrm{~A}$ & $2 \mathrm{CU} 6 \mathrm{~A}$ & $2 \mathrm{~F} 2 \mathrm{HA}$ & 1YRRA & $1 \mathrm{XV} 2 \mathrm{~A}$ & $1 \mathrm{H} 2 \mathrm{VC}$ \\
\hline 1EKJA & $1 \mathrm{M} 1 \mathrm{HA}$ & 1SR4A & 2PULA & 2ES9A & 1PN2A & $2 \mathrm{PV} 7 \mathrm{~A}$ & $1 \mathrm{AOCA}$ & $1 \mathrm{D} 20 \mathrm{~A}$ \\
\hline $1 \mathrm{KWAA}$ & $215 \mathrm{NM}$ & 2 FNOA & $1 \mathrm{VC} 1 \mathrm{~A}$ & 1TE5A & $1 \mathrm{G} 5 \mathrm{HA}$ & 2FEAA & 1S1QA & 1OEYJ \\
\hline 1DAAA & 1M5WA & 2PIAA & 1TKIA & $1 \mathrm{~K} 32 \mathrm{~A}$ & 1PUCA & $3 \mathrm{CNVA}$ & 1RLJA & 10EYA \\
\hline 1JZTA & 2D7VA & 1NBAA & 1 CSNA & 1SGMA & 2P5MA & $3 C W 9 A$ & 1OFDA & 1G3QA \\
\hline 1T6NA & 1JBSA & $3 D C X A$ & $1 \mathrm{MBMA}$ & $1 \mathrm{~L} 2 \mathrm{WA}$ & 2AEEA & 1DQAA & 1SZWA & 1EKEA \\
\hline 1SVMA & 1VQZA & 1EXOA & $1 X I Z A$ & $1 \mathrm{~L} 2 \mathrm{WI}$ & 1ITWA & 2OAFA & 10TKA & $2 \mathrm{HKUA}$ \\
\hline 2PSPA & 2HLJA & $1 \mathrm{~V} 6 \mathrm{ZA}$ & $104 G A$ & $1 \mathrm{~S} 12 \mathrm{~A}$ & 1JRLA & $1 \mathrm{FP} 3 \mathrm{~A}$ & 20WPA & $1 \mathrm{XVHA}$ \\
\hline 1Q7FA & 1VLFM & $2 \mathrm{D} 00 \mathrm{~A}$ & $1 \mathrm{GL} 4 \mathrm{~A}$ & 1RIFA & $1 \mathrm{~J} 5 \mathrm{WA}$ & 1SR9A & $1 \mathrm{COZA}$ & 1D3BB \\
\hline 1GVNA & $1 \mathrm{VLFN}$ & 1DP4A & 1B9HA & 1R7LA & 10GDA & $2 \mathrm{HKJA}$ & 1D3YA & 2FKZA \\
\hline $2034 \mathrm{~A}$ & 2IFXA & $1 \mathrm{HBKA}$ & 1YVIA & 2FNAA & 1OR7C & $1 \mathrm{FC} 3 \mathrm{~A}$ & $1 \mathrm{E} 8 \mathrm{CA}$ & $1 \mathrm{U} 5 \mathrm{KA}$ \\
\hline 1SA3A & $1 \mathrm{~F} 61 \mathrm{~A}$ & $1 \mathrm{P} 42 \mathrm{~A}$ & 1HE1A & 1ROVA & $1 \mathrm{~W} 94 \mathrm{~A}$ & 2LIGA & $2 \mathrm{BM} 5 \mathrm{~A}$ & 10R7A \\
\hline 2FIUA & 2HEKA & 2CZVA & 1U9LA & 217NA & 1I0RA & $1 \mathrm{~J} 3 \mathrm{WA}$ & 3E9VA & \\
\hline 2ARCA & $1 \mathrm{NN} 5 \mathrm{~A}$ & 2CZVC & 1D2TA & 1RYP2 & 1D7PM & 2OFYA & 1GTTA & $1 \mathrm{~F} 5 \mathrm{NA}$ \\
\hline 2CYJA & 2HQSA & $1 S 14 \mathrm{~A}$ & 1FOKA & 1RYP1 & $1 \mathrm{PX} 5 \mathrm{~A}$ & 1ZPSA & 1VP6A & $1 \mathrm{~F} 3 \mathrm{UB}$ \\
\hline 1V5IB & 2HQSC & 2I9FA & $1 \mathrm{E} 30 \mathrm{C}$ & 1RYPL & 1Z4RA & 1MVEA & 1R1TA & $1 \mathrm{~F} 3 \mathrm{UA}$ \\
\hline 1BTEA & 1PVMA & $1 \mathrm{G} 7 \mathrm{SA}$ & 2FBLA & 1RYPJ & 1NQUA & $10 A 8 \mathrm{~A}$ & $2 \mathrm{HAZA}$ & 1VR9A \\
\hline 1GUTA & 206PA & 1UQTA & $117 N A$ & 1RYPK & 3BLZA & 2AEUA & $1 \mathrm{~V} 71 \mathrm{~A}$ & $1 \mathrm{SG} 6 \mathrm{~A}$ \\
\hline $1 \mathrm{M} 6 \mathrm{KA}$ & 2PA7A & $1 Z C 3 B$ & $10 I S A$ & $1 \mathrm{Q} 2 \mathrm{HA}$ & 1TOTV & $2 \mathrm{~B} 5 \mathrm{GA}$ & 1MK4A & $2 \mathrm{BBKH}$ \\
\hline 2ZDPA & 1V5VA & $1075 \mathrm{~A}$ & 1SEIA & 2BF5A & 3B5EA & $1 \mathrm{JX} 4 \mathrm{~A}$ & 1UPTB & 2BBKL \\
\hline $1 \mathrm{FJ} 2 \mathrm{~A}$ & 1SH8A & 1UW4B & 1ETXA & 1GUDA & 1JYOA & $1 \mathrm{EZ3A}$ & 2DSTA & 1ELKA \\
\hline 2O1QA & 1EJ0A & 1TU1A & 1J5PA & $1 \mathrm{GXYA}$ & 1JYOE & $3 C \times 51$ & $2 \mathrm{GA} 1 \mathrm{~A}$ & 1V6SA \\
\hline
\end{tabular}




\begin{tabular}{|l|l|l|l|l|l|l|l|l|}
\hline 1MLAA & 1F46A & 1UW4A & 1R0DA & 1D0QA & 1YG6A & 3CX5C & 3CX5B & 1G2QA \\
\hline 2BAYA & 1A4IA & 1RQJA & 1K3SA & 1JMKC & 1C8UA & 1R5LA & 3CX5D & 1PUFB \\
\hline 2BMWA & 1INLA & 1VI6A & 2V94A & 2PBKA & 2GSVA & 2ZGWA & 3CX5F & 1N2AA \\
\hline 2I53A & 1H32A & 1RKTA & 1SQWA & 1ZRUA & 1C7NA & 2H6FB & 3CX5G & 1EERB \\
\hline 1L7AA & 1CS1A & 2NZCA & 1Y60A & 2F1NA & 1Q8RA & 2H6FA & 3CX5H & 3CX5A \\
\hline 2ASBA & 1DQZA & 2QWXA & 1DFMA & & & & & \\
\hline
\end{tabular}

